\title{
Rewriting the Rules Governing High Intensity Interactions of Light with Matter
}

\author{
Alex B. Borisov ${ }^{1}$, John C. McCorkindale ${ }^{1}$, Sankar Poopalasingam ${ }^{1}$, James W. Longworth ${ }^{1}$, Peter Simon ${ }^{2}$, Sándor \\ Szatmári ${ }^{3}$, and Charles K. Rhodes ${ }^{1}$ \\ ${ }^{1}$ Laboratory for X-Ray Microimaging and Bioinformatics, Department of Physics, University of Illinois at Chicago, \\ Chicago, IL 60607-7059, USA \\ ${ }^{2}$ Laser-Laboratorium Göttingen e.V. \\ Hans-Adolf-Krebs-Weg 1, D-37077 Göttingen, Germany \\ ${ }^{3}$ Department of Experimental Physics, University of Szeged \\ Dóm tér 9, H-6720 Szeged, Hungary
}

\begin{abstract}
The trajectory of discovery associated with the study of high-intensity nonlinear radiative interactions with matter and corresponding nonlinear modes of electromagnetic propagation through material that have been conducted over the last 50 years can be presented as a landscape in the Intensity/Quantum Energy [I- $\hbar \omega]$ plane. Based on an extensive series of experimental and theoretical findings, a universal zone of anomalous enhanced electromagnetic coupling, designated as the Fundamental Nonlinear Domain, can be defined. Since the lower boundaries of this region for all atomic matter correspond to $\hbar \omega \sim 10^{3} \mathrm{eV}$ and $\mathrm{I} \approx 10^{16} \mathrm{~W} / \mathrm{cm}^{2}$, it heralds a future dominated by $\mathrm{x}$-ray and $\gamma$-ray studies of all phases of matter including nuclear states. The augmented strength of the interaction with materials can be generally expressed as an increase in the basic electromagnetic coupling constant in which the fine structure constant $\alpha \rightarrow Z^{2} \alpha$, where $Z$ denotes the number of electrons participating in an ordered response to the driving field. Since radiative conditions strongly favoring the development of this enhanced electromagnetic coupling are readily produced in self-trapped plasma channels, the processes associated with the generation of nonlinear interactions with materials stand in natural alliance with the nonlinear mechanisms that induce confined propagation. An experimental example involving the $\mathrm{Xe} 4 \mathrm{~d}$-shell for which $\mathrm{Z} \cong 18$ that falls in the specified anomalous nonlinear domain is described. This yields an effective coupling constant of $Z^{2} \alpha \cong 2.4>1$, a magnitude comparable to the strong interaction and a value rendering as useless conventional perturbative analyses founded on an expansion in powers of $\alpha$. This enhancement can be quantitatively understood as a direct consequence of the dominant role played by coherently driven multiply-excited states in the dynamics of the coupling. It is also conclusively demonstrated by an abundance of data that the utterly peerless champion of the experimental campaign leading to the definition of the Fundamental Nonlinear Domain was Excimer Laser Technology. The basis of this unique role was the ability to satisfy simultaneously a triplet $(\omega, \mathrm{I}, \mathrm{P})$ of conditions stating the minimal values of the frequency $\omega$, intensity $\mathrm{I}$, and the power P necessary to enable the key physical processes to be experimentally observed and controllably combined. The historical confluence of these developments creates a solid foundation for the prediction of future advances in the fundamental understanding of ultra-high power density states of matter. The atomic findings graciously generalize to the composition of a nuclear stanza expressing the accessibility of the nuclear domain. With this basis serving as the launch platform, a cadenza of three Grand Challenge Problems representing both new materials and new interactions is presented for future solution; they are (1) the performance of an experimental probe of the properties of the vacuum state associated with the Dark Energy at an intensity approaching the Schwinger/Heisenberg Limit, (2) the attainment of amplification in the $\gamma$-ray region $(\sim 1 \mathrm{MeV})$ and the discovery of a nuclear excimer, and (3) the determination of a path to the projected super-heavy Nuclear Island of Stability.
\end{abstract}




\section{Contents}

1. Introduction

2. Experimental Definition of the Fundamental Nonlinear Domain

2.1 Intensity - Frequency Interaction Landscape:

Experimentally Determined Zone of Anomalous Coupling

2.2 Experimental Confirmation of Fundamental Nonlinear Domain with Observation of Enhanced Nonlinear Coupling in the X-Ray Range

2.2.1. Experimental Evidence

2.2.2. Analysis of the $X e(M) \rightarrow X e(L) 5 \gamma$ Cross Section $\sigma_{5}$ 2.2.2.1 Formulation of Estimated Cross Section 2.2.2.2 Fundamental Ansatz

3. Femtosecond UV $248 \mathrm{~nm}$ High-Brightness Laser Technology

3.1 Introduction

3.2 Basic Femtosecond Excimer Laser Properties and Design Logic

3.3 Short-pulse Amplification Properties of Excimers

3.3.1 Gain Dynamics

3.3.2 Amplification in Media having Non-Saturable Absorption

3.3.3 Off-Axis and Multiple-Pass Off-Axis Amplification Geometries

3.3.4 Interferometric Multiplexing

3.4 Pulse Cleaning Methods

3.5 Power and Energy Scalability of High-Brightness Discharge Pumped

Excimer Amplifier Systems

4. Grand Challenge Problems

4.1 Nuclear Excitation Preamble/Analogy with Atomic Inner-Shell Excitation

4.1.1 Atomic Inner-Shell Excitation

4.1.2 Extension to Direct Nuclear Excitation

4.2 Discussion of Grand Challenge Problems

4.2.1 Direct Probe of the Vacuum State

4.2.2 Amplification in the Gamma-Ray Region

4.2.3 Path to Nuclear Super-Heavy Island of Stability

5. Conclusions

Acknowledgements

References

Supplementary Material 


\section{Introduction}

The history of nonlinear high-intensity interactions, that commenced in 1961 with the observation of second harmonic radiation [1] at $347.2 \mathrm{~nm}$ in crystalline quartz, records a span of $\sim 10^{18}$ in experimental intensity that enabled the discovery of an expansive range of entirely new phenomena. As a consequence of a multitude of salient advances, this general field of inquiry remains a stable and highly robust province of fundamental laser-based research after a half century. Empowered by many developments in short wavelength generation [2-11], and assisted particularly by key results stemming from the dynamics of nonlinear modalities of confined propagation [12-26], the study of broad classes of nonlinear radiative phenomena now steps up into the x-ray spectral region, an advance that perforce lifts the interactions to a new and enormously increased level of power density. The present work discusses the nature of this transition, gives an explicit experimental example that represents a defining characteristic of future research activity, and concludes with the statement of three Grand Challenge Problems for prospective solution.

\section{Experimental Definition of the Fundamental Nonlinear Domain}

\subsection{Intensity - Frequency Interaction Landscape: Experimentally Determined Zone of Anomalous Coupling}

Founded on the analysis of a long sequence of experimental and theoretical studies [27-67], it is possible to identify a zone of anomalous enhanced electromagnetic coupling that stands in sharp contrast to a corresponding region of conventionally described weaker interaction. These two areas can be presented in the intensity $(I)$-quantum energy $(\mathrm{h} \omega)$ plane, as illustrated in Fig.(1), and the boundaries between these zones designate the transition to a fundamentally new regime of physical interaction that stands as the green shaded quadrant in the upper right. We will find below that the picture presented in Fig. (1) can be sharpened to be a universal statement about all atomic matter, a refinement that modestly reshapes the zone shown by raising the lower quantum energy bound to $\hbar \omega$ $\sim 1 \mathrm{keV}$.

Extant data are used below to establish the boundaries of the anomalous region that we designate as the Fundamental Nonlinear Domain in Fig.(1). Stating the conclusion of the analysis at the outset, it is found that lower limit of the intensity for the enhanced region of coupling shown in Fig.(1) is given approximately by $\mathrm{I}: 3 \times 10^{15}-10^{16} \mathrm{~W} / \mathrm{cm}^{2}$, a narrow range of values corresponding to an electric field slightly less than one atomic unit $\left(\mathrm{e} / a_{o}^{2}: 5.14 \times 10^{9} \mathrm{~V} / \mathrm{cm}\right)$. The corresponding lower bound on the frequency $\omega$, estimated on the basis of measurements with $\mathrm{N}_{2}, \mathrm{Kr}, \mathrm{Xe}$, and $\mathrm{U}$, is represented by $\mathrm{h} \omega: 5 \mathrm{eV}-6 \mathrm{eV}$, a magnitude slightly less than one half of a Rydberg. Therefore, as a gross overall measure, the region of anomalous interaction is physically bounded by the characteristic values of electric field strength (intensity) and frequency (energy) expressed by the structure of the hydrogen atom, the paramount fundamental atomic entity.

The main region of interest, as developed below, involves the $\mathrm{x}$-ray range with quantum energies $\mathrm{h} \omega \geq 10^{3} \mathrm{eV}$ and intensities $\mathrm{I} \geq 10^{16} \mathrm{~W} / \mathrm{cm}^{2}$, since this terrain evidences properties that enable it to uniformly and universally reflect the anomalous interaction for all materials regardless of their atomic constituents or physical state. Radiative coupling in this zone will be completely dominated by anomalous enhanced coupling strengths that will be particularly prominent for high-Z 
materials $[27,30,46,49]$. In the description below, new data are presented that confirm the existence of this predicted zone through measurements made in the $\mathrm{keV}$ region with xenon $(\mathrm{Z}=54)$.

We now describe the experimental history that enabled the construction of the physical map shown in Fig.(1) and the placement of the boundaries defining the Fundamental Nonlinear Domain. The story commences in the infrared. The discovery of the $\mathrm{CO}_{2}$ laser [68] in 1964 with a wavelength $\lambda \sim 10 \mu \mathrm{m}$ enabled the development of new high-resolution spectroscopic techniques that could be readily performed on infrared molecular vibrational-rotational transitions. These methods combined very high experimental precision with intensities sufficiently elevated to observe nonlinear twophoton $(2 \gamma)$ amplitudes quantitatively. An example is given by the detailed measurements [69] that were conducted in 1976 on specific vibrational-rotational transitions of the $v_{2}$-mode of ${ }^{14} \mathrm{NH}_{3}$. The quantitative analysis of both the $2 \gamma$ coupling strength and the intensity dependent shift of the resonance observed in these measurements demonstrated solid agreement with a conventional perturbative analysis. The datum associated with this study is placed on Fig.(1) with the identification $\left[\mathrm{NH}_{3}(2 \gamma)\right]$. 


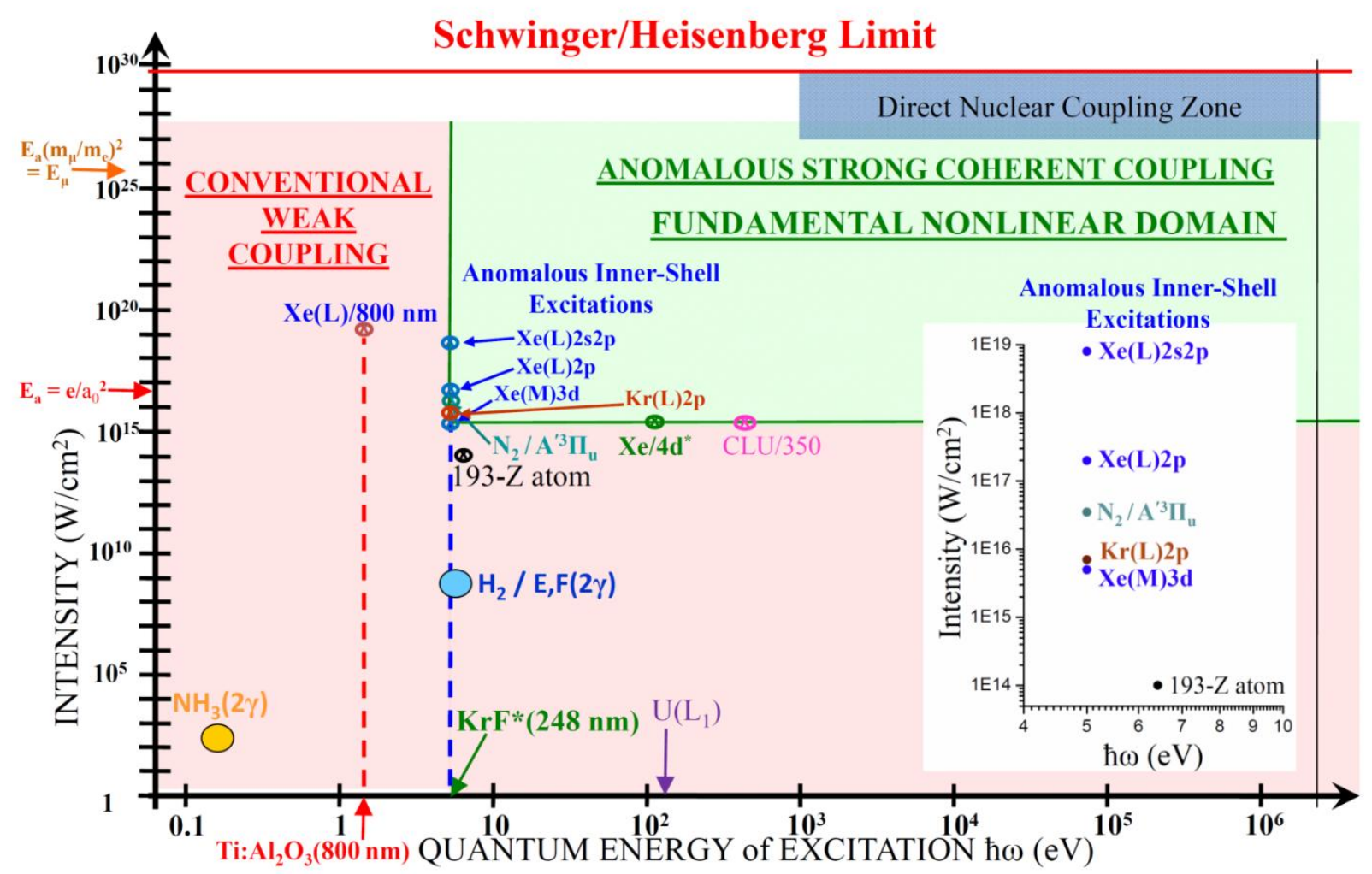

Fig. (1): Global Physical Scaling in the Intensity-Quantum Energy Plane. Based on the series of studies at different intensities and wavelengths, the $I-\hbar \omega$ plane is partitioned into two separate regions, one associated with conventional weak electromagnetic coupling (red) and a second (green) designating the Fundamental Nonlinear Domain that is characterized by an anomalous enhanced coupling strength. Several specific experimental studies described in the text are indicated. A prospectively identified Direct Coupling Nuclear Zone is shown in the region bounded by $\mathrm{I} \geq 10^{27}$ $\mathrm{W} / \mathrm{cm}^{2}$ and $\hbar \omega \geq 10^{3} \mathrm{eV}$. The $\mathrm{U}\left(\mathrm{L}_{1}\right)$ Auger width at $\sim 124 \mathrm{eV}$ is marked on the abscissa. The intensities corresponding to field strengths of an atomic unit $\mathrm{E}_{\mathrm{a}}=\mathrm{e} / \mathrm{a}_{0}{ }^{2}$ and its muonic counterpart $\mathrm{E}_{\mathrm{a}}\left(\mathrm{m}_{\mu} / \mathrm{m}_{\mathrm{e}}\right)^{2}$ are marked on the ordinate for reference. The Schwinger/Heisenberg Limit determines the upper boundary at $\mathrm{I} \cong 4.6 \times 10^{29} \mathrm{~W} / \mathrm{cm}^{2}$. The inset illustrates the ascending series of inner-shell thresholds discussed in the text that define the location of the vertical boundary at $\hbar \omega \cong 5 \mathrm{eV}$.

The invention of the $\operatorname{KrF}^{*}(248 \mathrm{~nm})$ and $\operatorname{ArF}^{*}(193 \mathrm{~nm})$ excimer laser systems [70, 71] in 1975 enabled the extension of the quantitative nonlinear spectroscopic methods successfully demonstrated in the infrared to the ultraviolet spectral range. The first examples of high resolution spectroscopy utilizing nonlinear coupling at an ultraviolet wavelength $(193 \mathrm{~nm})$ were the studies in 1978 and 1980 of the $2 \gamma$ excitation $[72,73]$ of the double-minimum E,F ${ }^{1} \Sigma_{\mathrm{g}}{ }^{+}$state of $\mathrm{H}_{2}$, a level that possesses the same symmetry as the ground $\mathrm{X}^{1} \Sigma_{\mathrm{g}}{ }^{+}$term. Accordingly, the $\mathrm{X} \rightarrow \mathrm{E}, \mathrm{F}$ excitation is strictly forbidden as a single-photon amplitude. The quantitative analysis of these measurements again clearly showed that the measured coupling strength conformed well to a conventional perturbative treatment for a second order process. The matrix elements and state energies of $\mathrm{H}_{2}$ are well known, so that the effective cross section could be reliably computed and the presence of a significant anomalous component of 
the interaction could be ruled out. This study is designated on Fig. (1) as the datum $\left[\mathrm{H}_{2} / \mathrm{E}, \mathrm{F}(2 \gamma)\right]$ at an intensity of $\sim 10^{9} \mathrm{~W} / \mathrm{cm}^{2}$.

The subsequent development of short-pulse excimer laser technology [74] in the picosecond range ( 10 ps) in 1982 immediately enabled the attainment of sharply elevated intensities at an ultraviolet wavelength. Specifically, electric field strengths at values corresponding approximately to one tenth of an atomic unit $\left(\sim \mathrm{e} / 10 a_{o}^{2} \approx 5 \times 10^{8} \mathrm{~V} / \mathrm{cm}\right)$ could be achieved. The initial experimental studies conducted with an $\mathrm{ArF}^{*}(193 \mathrm{~nm})$ source immediately gave an indication of anomalous coupling; this work produced the first hint of an unconventional and substantially enhanced strength of interaction. These measurements analyzed the atomic number $(\mathrm{Z})$ dependence of collision-free multiple ionization of atoms [27] with $193 \mathrm{~nm}$ radiation $(\hbar \omega \approx 6.3 \mathrm{eV})$ at an intensity of $\sim 10^{14} \mathrm{~W} / \mathrm{cm}^{2}$. The outcome was highly significant, since the customary theoretical approaches clearly could not describe the chief feature of the observations, specifically, the production of stages of ionization far above the conventional predictions. On the basis of these results, it was concluded (1) that conventional treatments of multiquantum ionization did not correspond to the experimental findings for high- $\mathrm{Z}$ materials and (2) that the atomic shell structure was an important factor in determining the magnitude of the coupling. Furthermore, based on the Z-dependence of the experimental results, a collective motion of the $\mathrm{d}$ and $\mathrm{f}$ shells, essentially a plasma model, that can be imagined as the atomic counterpart of the nuclear giant dipole [75], was inferred [27]. In the cases of I and Xe, the experimental evidence pointed particularly to a role played by the $4 \mathrm{~d}$-shell. This study is denoted on Fig.(1) as [193-Z atom] at an intensity of $\sim 10^{14} \mathrm{~W} / \mathrm{cm}^{2}$, a value that is a factor of $\sim 10^{5}$ greater than that used in the studies of the E,F $\Sigma_{\mathrm{g}}{ }^{+}$state of the hydrogen molecule described above. In time additional results appeared $[35,50,52,53,59,61]$ that supported this conclusion.

Elaboration on the nature of these possible ordered electronic motions appeared [28-30,76] shortly thereafter, two involving analogies with fast atom-atom collisions $[28,76]$. The basic idea can be summarized as an ordered electronic motion that achieves a "phase space control" of the energy. A simple picture aimed at the evaluation of an upper bound $\sigma_{\mathrm{m}}$ of the effective nonlinear cross section in the high- $Z$ and high-intensity limit was developed [46] that predicted the existence of a universal limiting value of

$$
\sigma_{m} \approx 8 \pi \mathrm{D}_{c}^{2} \approx 3.6 \times 10^{-20} \mathrm{~cm}^{2}
$$

that would be approached for intensities $>10^{20} \mathrm{~W} / \mathrm{cm}^{2}$. The universality of Eq.(1) is astounding; it depends only upon the electron mass through its Compton wavelength $\mathrm{D}_{c}$ and is completely independent of the conventional notion of the nonlinear order of the amplitude, the electronic charge, the frequency of the radiation, and any atomic properties. In addition, the magnitude of $\sigma_{\mathrm{m}}$ was highly significant, since it indicated that specific power densities greater than $\sim 1$ W/atom could be possible as high-intensity laser technology continued to develop and enable the attainment of experimental intensities in the $\sim 10^{20} \mathrm{~W} / \mathrm{cm}^{2}$ range. Experimental intensities above this level are now routinely produced in laboratories worldwide.

The next major development was the introduction of a molecular concept and the study of a suitable model system at an intensity sufficiently high to reveal the anomalous coupling in a dominant 
manner. Studies of $\mathrm{N}_{2}$ with subpicosecond $248 \mathrm{~nm}$ radiation in 1989 at intensities in the $10^{16}-10^{17}$ $\mathrm{W} / \mathrm{cm}^{2}$ range, for which the optical electric field is approximately one atomic unit (e/ $\left.a_{o}^{2}\right)$, quickly provided the necessary data [32]. The key observation was the selective production of a molecular inner-shell $2 \sigma_{\mathrm{g}}$ vacancy that was detected by its characteristic radiative decay at $\lambda \sim 55.8 \mathrm{~nm}$. The detection of this line was revelatory on the dynamics of the interaction. Specifically, it led unexpectedly to the identification [32] of a new previously unknown state $\left(\mathrm{A}^{\prime}{ }^{3} \Pi_{\mathrm{u}}\right)$ of $\mathrm{N}_{2}{ }^{2+}$ and the unambiguous assignment [32] of the $55 \mathrm{~nm}$ emission to the $A^{\prime}{ }^{3} \Pi_{u} \rightarrow D^{3} \Pi_{g}$ transition of $N_{2}{ }^{2+}$. Hence, the strong generation of the $55 \mathrm{~nm}$ line clearly demonstrated that it was possible to produce an innershell vacancy without simultaneously removing a large fraction of the outer shell electrons. An electronically hollow system was the prompt result of the excitation.

The radiative transition observed at $\lambda \sim 55.8 \mathrm{~nm}$ involving the $2 \sigma_{\mathrm{g}}$ vacancy likewise possessed several highly unusual properties. Although a fully electric dipole (E1) allowed amplitude and the most intense feature in the recorded spectrum [32], this line was unknown and had never been reported during approximately a century of spectroscopic study under conditions that had utilized a wide range of methods for excitation of this elementary homonuclear diatomic molecule [77, 78]. This puzzling observation, in an extensively studied simple system, in alliance with the high intensity of the $55 \mathrm{~nm}$ emissions, was doubly significant. These results signified (1) that special conditions were required to produce the particular excited state that was the source of the $55.8 \mathrm{~nm}$ emission and (2), if those conditions were met, efficient production of the $\mathrm{A}^{\prime}{ }^{3} \Pi_{\mathrm{u}}$ state was the outcome, an indication that the channel was dynamically favored. The conclusion was clear; inner-shell excited states could be selectively and efficiently produced in molecules.

The analysis [32] of the observed spectrum demonstrated that these exceptional characteristics were matched by corresponding unexpected and unusual properties of the upper $\mathrm{A}^{\prime}{ }^{3} \Pi_{\mathrm{u}}$ level and the electronic character of the $\mathrm{A}^{\prime} \rightarrow \mathrm{D}$ transition. The excited $\mathrm{A}^{\prime}$ level was found to be a bound "charge transfer" state whose asymptote correlates with the charge asymmetric $\mathrm{N}^{2+}+\mathrm{N}$ configuration; the polarization-attraction of the $\mathrm{N}^{2+}$ ion to the neutral $\mathrm{N}$ atom accounts for the binding. In contrast, the lower $\mathrm{D}^{3} \Pi_{\mathrm{g}}$ state is well known and correlates to the obviously repulsive unbound symmetric $\mathrm{N}^{+}+\mathrm{N}^{+}$ asymptote. Therefore, the $\mathrm{A}^{\prime} \rightarrow \mathrm{D}$ transition at $\lambda \sim 55.8 \mathrm{~nm}$ is "excimer-like" and corresponds to an electronic motion that accomplishes the transfer of an electron from the neutral $(\mathrm{N})$ end of the molecule to the doubly charged $\left(\mathrm{N}^{2+}\right)$ side. It follows that the production of the $\mathrm{A}^{\prime}{ }^{3} \Pi_{\mathrm{u}}$ state involves the orderly motion of an electron induced by the external electric field along the axis of the molecule in the opposite direction. With an interatomic spacing $\mathrm{d} \approx 1.1 \AA$ and an electric field of $\sim 5.1 \times 10^{9}$ $\mathrm{V} / \mathrm{cm}$, the work $\mathrm{W}$ associated with this electron transfer is

$$
\mathrm{W} \approx \mathrm{eEd} \approx 56 \mathrm{eV}
$$

a magnitude sufficient to liberate a $2 \sigma_{\mathrm{g}}$ electron whose binding energy $[77,79]$ is $\sim 37.7 \mathrm{eV}$. The point on Fig.(1) denoted as $\left[\mathrm{N}_{2} / \mathrm{A}^{\prime}{ }^{3} \Pi_{\mathrm{u}}\right]$ represents this experimental study of $\mathrm{N}_{2}$.

The chief conclusion derived from the experiments on $\mathrm{N}_{2}$ was that ordered motions of electrons could be induced in molecules that can efficiently and selectively remove inner-orbital electrons while leaving outer-shell electrons largely undisturbed. Hence, the combination (1) of this induced ordered motion with (2) the collective plasma picture associated with sufficiently high-Z 
atoms, that was supported by the studies of multiphoton ionization [27-30] described above, presented itself as an obvious possibility for efficient short wavelength generation. Specifically, with these molecular dynamics, the use of a high- $Z$ molecule could enable the scaling of the inner-shell excitation observed in $\mathrm{N}_{2}$ with the $2 \sigma_{\mathrm{g}}$ orbital at $\sim 40 \mathrm{eV}$ to far more deeply bound corresponding states in the multikilovolt range [33].

At this stage in the development of the study of high-intensity interactions, a new centrally important nonlinear optical phenomenon was experimentally established that enabled the stable compression of power in macroscopic volumes be achieved. The initial demonstration [12] of selftrapped channeling of $248 \mathrm{~nm}$ radiation in 1992 has been recently extended with a quantitative experimental comparison of channels produced in $\mathrm{Kr}$ and $\mathrm{Xe}$ cluster targets [26,80]. The availability of this physical mechanism for power compression had a profound influence on the study of nonlinear processes, since it enabled three conditions to be simultaneously and simply produced [14$16,18,20,24]$. They are (1) the production of intensities in the $10^{20}-10^{21} \mathrm{~W} / \mathrm{cm}^{2}$ region, (2) a sharp rise time of the propagating pulse of $\sim 3$ fs that is perforce generated by the dynamics of channel formation, and (3) a greatly extended length of the high intensity region provided by the channel that readily reaches several millimeters.

The visualization of these channels, as shown in Fig.(2) for the case of Xe, illustrates many properties of the self-trapped propagation [26,80]. The comparison of the Thomson image of the electron density with the simultaneously recorded transversely observed morphology of the $\mathrm{Xe}(\mathrm{M}) \mathrm{X}$ ray $(\hbar \omega \sim 1 \mathrm{keV})$ emission zone, as presented in Fig.(2), is revealing. The correspondences between the Thomson image in panel (a) and the $\mathrm{Xe}(\mathrm{M}) \mathrm{x}$-ray image in panel (b) manifestly show that the detailed channel dynamics at the position $\mathrm{Z} \cong 0 \mathrm{~mm}$, where the large abrupt transverse expansion occurs, clearly signal in both images the termination of the confined propagation and the concomitant release of the trapped $248 \mathrm{~nm}$ pulse energy from the channel. The collapsed narrow zone of the channel observed before this terminus has a length $\ell_{\mathrm{Xe}} \cong 0.8 \mathrm{~mm}$. Furthermore, in this compressed region between $-0.8 \mathrm{~mm} \leq \mathrm{Z} \leq 0.0 \mathrm{~mm}$, the small structural features visible in the Thomson recording are directly mirrored by matching variations in the x-ray emission at the exact corresponding axial positions in the $\mathrm{x}$-ray image. The intensity in this region is estimated to be $\sim 10^{20} \mathrm{~W} / \mathrm{cm}^{2}$ with an atom-specific power density approaching $\sim 1 \mathrm{~W} /$ atom. 


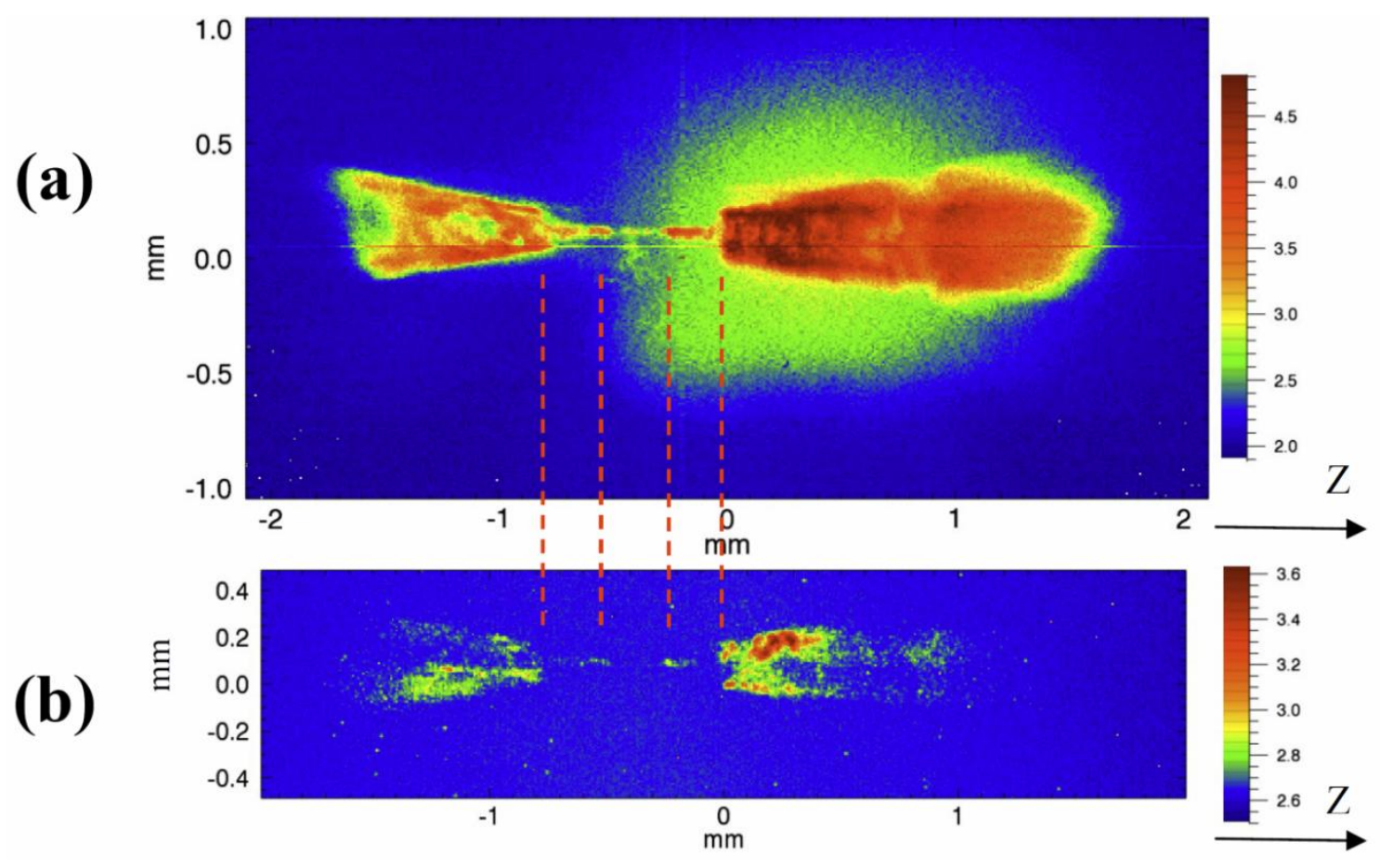

Fig. (2): Simultaneously recorded single-pulse images of (a) the Thomson scattered signal from the electron density and (b) the transverse $\mathrm{Xe}(\mathrm{M}) \sim 1 \mathrm{keV}$ x-ray emission zone (log scale) of a stable 248 $\mathrm{nm}$ channel produced in a Xe cluster target. Experimental parameters: pulse energy $217 \mathrm{~mJ}, \mathrm{Xe}$ plenum pressure $185 \mathrm{psi}$, and Xe plenum temperature $285 \mathrm{~K}$. The X-ray camera utilized a pinhole with a diameter of $10 \mu \mathrm{m}$, a size that gives a limiting spatial resolution estimated to be $20-30 \mu \mathrm{m}$. The direction of propagation is left to right and the center of the nozzle corresponds to the coordinate $\mathrm{Z} \cong 0 \mathrm{~mm}$. The Xe cluster target was produced by a cooled high-pressure pulsed-valve fitted with a circular nozzle having a diameter of $2.5 \mathrm{~mm}$. These data correspond to pulse \#19 (24 January 2011). The locations of corresponding features in these images are indicated by the vertical connecting lines. The sharp expansion at $\mathrm{Z} \cong 0 \mathrm{~mm}$ in panel (a) signals the termination of the self-trapped channel. A broad elliptical halo of ionization with a diameter of $\sim 1 \mathrm{~mm}$ is seen in the $-0.5 \mathrm{~mm} \leq \mathrm{Z} \leq 1.0 \mathrm{~mm}$ region. The abrupt expansion of the $\mathrm{x}$-ray emission in panel (b) at $\mathrm{Z} \cong 0 \mathrm{~mm}$ marking the end of the channeled propagation mirrors the corresponding morphology of the Thomson image in panel (a) and the localized zones of $\mathrm{x}$-ray emission coincide with matching features in the Thomson image. Figure adapted from Ref.[26] and used with permission. 
The concept of the high-Z molecular system [33] was tested in 1994 with $\mathrm{Kr}$ and Xe clusters $[34,81]$ and the effectiveness of the molecular dynamics was immediately apparent. The key findings were (1) the copious generation of multikilovolt $\mathrm{X}$-rays on $\mathrm{Kr}(\mathrm{L}), \mathrm{Xe}(\mathrm{M})$, and $\mathrm{Xe}(\mathrm{L})$ transitions involving $2 \mathrm{p}$ and $3 \mathrm{~d}$ vacancies spanning the $\sim 1-4.5 \mathrm{keV}$ range, (2) the discovery that the $\mathrm{Xe}(\mathrm{L})$ emission corresponded exclusively to hollow atom $\mathrm{Xe}^{\mathrm{q}+}$ charge state arrays $[34,38]$ for $30 \leq \mathrm{q} \leq 36$, and (3) the observation of an exceptional brightness of the recorded emission that clearly signaled the action of an anomalously enhanced coupling strength [35,52,53,82]. In 1995, the cluster mechanism and the self-trapped channels were successfully combined [37], an important result that demonstrated the mutual compatibility of these two highly nonlinear processes. The importance of the clusters was underscored, since an earlier study on Xe atoms [83] unambiguously demonstrated that $\mathrm{Xe}(\mathrm{M})$ and $\mathrm{Xe}(\mathrm{L}) \mathrm{x}$-rays were not produced by ultraviolet radiation at the intensities used in the cluster studies [34,81]. The cluster formation was essential in order to involve the molecular dynamics, initially observed in $\mathrm{N}_{2}$ [32], that produced the strong efficient excitation of the $\mathrm{A}^{\prime}{ }^{3} \Pi_{\mathrm{u}}$ state of $\mathrm{N}_{2}{ }^{2+}$, and enabled the generation of the deep $2 \mathrm{p}$ and $3 \mathrm{~d}$ inner-shell excitations. In addition, the direct hollow atom production [34] was a huge bonus, since it eliminated the need for the relatively slow process of recombination to form the excited $\mathrm{Xe}^{*}(\mathrm{~L})$ states. Hence, excited levels exhibiting prompt x-ray emission were efficiently produced, an outcome that automatically produced inverted population densities and subsequently enabled amplification in the multikilovolt $\mathrm{x}$-ray spectral region $\sim 4.5 \mathrm{keV}$ on $3 \mathrm{~d} \rightarrow 2 \mathrm{p}$ transitions to be achieved [38,39,61-63,84]. Recent work [85] has extended these findings with the demonstration of amplification on the $\operatorname{Kr}(\mathrm{L}) 3 \mathrm{~s} \rightarrow 2 \mathrm{p} \mathrm{Kr}^{26+}{ }^{1} \mathrm{P}_{1} \rightarrow{ }^{1} \mathrm{~S}_{0}$ line at $\lambda \cong 7.5$ $\AA$.

In a series of related studies, several centrally important properties of the $\operatorname{Kr}(\mathrm{L}), \mathrm{Xe}(\mathrm{M})$, and $\mathrm{Xe}(\mathrm{L})$ emissions were experimentally established. Among these were the threshold intensities under $248 \mathrm{~nm}$ irradiation for $\mathrm{Kr}(\mathrm{L}) 2 \mathrm{p}, \mathrm{Xe}(\mathrm{M}) 3 \mathrm{~d}, \mathrm{Xe}(\mathrm{L}) 2 \mathrm{p}$, and $\mathrm{Xe}(\mathrm{L}) 2 \mathrm{~s} 2 \mathrm{p}$ vacancy production that are represented in Table I. These results are illustrated in the inset in Fig. (1) as a vertical series of points designated as $[\mathrm{Kr}(\mathrm{L}) 2 \mathrm{p}, \mathrm{Xe}(\mathrm{M}) 3 \mathrm{~d}, \mathrm{Xe}(\mathrm{L}) 2 \mathrm{p}, \mathrm{Xe}(\mathrm{L}) 2 \mathrm{~s} 2 \mathrm{p}]$ for the quantum energy $\mathrm{h} \omega=5 \mathrm{eV}$ at their respective intensities. A second important quantity determined was the efficiency of $\mathrm{Xe}(\mathrm{M})$ generation [26] and the magnitude of the X-ray yield that it could produce. The measurements demonstrated $>50 \mathrm{~mJ} /$ pulse on the $\mathrm{Xe}(\mathrm{M})$ band [50] and a corresponding efficiency of production $>$ $20 \%$ with $248 \mathrm{~nm}$ excitation. Since the $248 \mathrm{~nm} \mathrm{KrF}^{*}$ technology [86] is presently scalable to pulse energies of $\sim 2 \mathrm{~J}$ and the $\mathrm{Xe}(\mathrm{M})$ emission spectrum at $\hbar \omega \cong 1 \mathrm{keV}$ can be generated [26] with an efficiency of $\sim 30 \%$ and has a bandwidth [66] of $\sim 100 \mathrm{eV}$, the door is now open to the production of attosecond pulses of coherent x-ray pulses at the $\sim 500 \mathrm{~mJ}$ level [87], a point echoed in section 3 .

In sharp contrast to the $2 \mathrm{p}$ and $2 \mathrm{~s} 2 \mathrm{p}$ inner-shell excitations readily produced with ultraviolet radiation at $\mathrm{h} \omega=5 \mathrm{eV}$ with Xe clusters, the performance of directly comparable experiments with $\mathrm{Xe}$ at $800 \mathrm{~nm}(\mathrm{~h} \omega \sim 1.5 \mathrm{eV})$ produced no significant $\mathrm{Xe}(\mathrm{L})$ production [41,51]. The low level of $\mathrm{Xe}(\mathrm{L})$ emission detected had a far different spectral envelope and was quantitatively 3000-fold weaker. The point on Fig.(1) that designates the experiments performed with $800 \mathrm{~nm}$ excitation is denoted by $[\mathrm{Xe}(\mathrm{L}) / 800 \mathrm{~nm}]$. The net conclusion from the set of studies described above is that two factors were simultaneously essential for efficient inner-shell excitation; they are (1) the molecular environment provided by the cluster and (2) irradiation with a sufficiently high frequency.

These comparative measurements [41,51] of the $\mathrm{Xe}(\mathrm{L})$ spectra and corresponding $\mathrm{X}$-ray yields were a direct demonstration that the wavelength of excitation was an important factor in the 
interaction that produced the inner-shell $2 p$ vacancies. Indeed, an earlier analysis [30], based on an analogy with atom-atom collisions, had estimated the optimum frequency $\omega$ for excitation as

$$
\omega \cong 30 \alpha^{3} c / \mathrm{D}_{c}
$$

in which $\alpha$ denotes the fine structure constant and $\mathrm{D}_{c}$ is the reduced Compton wavelength. This relationship yields a wavelength of $\lambda \approx 200 \mathrm{~nm}$, a magnitude not far from the experimental values of $193 \mathrm{~nm}$ and $248 \mathrm{~nm}$ used in the studies of $\mathrm{N}_{2}$ and the $\mathrm{Kr}$ and $\mathrm{Xe}$ clusters described above $[32,34,38,81,85]$. It is also considerably shorter than the $800 \mathrm{~nm}$ wavelength used in the comparative studies $[41,51]$ that showed practically no x-ray generation.

These findings led to important insight on the mechanism of inner shell excitation. On the basis of several subsequent analyses [35,36,42,52], it was concluded that an ordered phase-dependent interaction was present. Furthermore, it was found that the experimental findings could be understood [42] if the $\mathrm{L}_{1}$-subshell Auger widths [88-96] were used as a measure of the dephasing time that destroyed the ordered motion. A new analysis, that explores the excitation of the $\mathrm{Xe}(\mathrm{L})$ hollow atom states in self-trapped plasma channels [12-26,40] and is presented in section 4.1.1 below, illustrates the dynamical details of the attosecond character of this interaction that are dependent on the confined propagation [26].

The observational pattern developed by this sequence of experimental studies was sufficient to establish prospectively two fundamental divisions in the $I-\hbar \omega$ plane. The main lesson delivered by the experimental findings discussed above was that, at sufficiently high intensity at a sufficiently short wavelength, an anomalously enhanced coupling strength governs the interaction. The ascending series of six points [193-Z atom, $\mathrm{Xe}(\mathrm{M}) 3 \mathrm{~d}, \mathrm{Kr}(\mathrm{L}) 2 \mathrm{p}, \mathrm{N}_{2} / \mathrm{A}^{\prime 3} \mathrm{\Pi}_{\mathrm{u}}, \mathrm{Xe}(\mathrm{L}) 2 \mathrm{p}$, and $\mathrm{Xe}(\mathrm{L}) 2 \mathrm{~s} 2 \mathrm{p}$ ] detailed in the inset place on Fig.(1) defines the vertical boundary at $\mathrm{h} \omega \sim 5 \mathrm{eV}$; the anomalous coupling observed is associated with frequencies $\mathrm{h} \omega>5 \mathrm{eV}$. A corresponding bifurcation associated with the ordinate of Fig.(1) can be placed at the intensity $\mathrm{I} \approx 5-7 \times 10^{15} \mathrm{~W} / \mathrm{cm}^{2}$ that is established by the cluster of points grouped near the $\operatorname{Xe}(\mathrm{M})[\mathrm{Xe}(\mathrm{M}) 3 \mathrm{~d}]$ and $\operatorname{Kr}(\mathrm{L})[\operatorname{Kr}(\mathrm{L}) 2 \mathrm{p}]$ thresholds. With the physical Ansatz that the minimum electric field required to develop the anomalous interaction is independent of the frequency, or equivalently, that a minimum level of force on the electrons must be exerted, the line marking this estimated transition was initially drawn horizontally with a vanishing slope. This assumption recalls the result given in Eq.(1) in which the value of the upper board of the cross-section $\sigma_{\mathrm{m}}$ in the high-intensity high-Z limit was found to be independent of the frequency $\omega$. This physical choice was subsequently confirmed by the results of two independent studies. One involved the ionization of clusters [45] in the soft $\mathrm{x}$-ray $(\mathrm{h} \omega=350 \mathrm{eV})$ regime and the other [44] concerned the excitation of the prominent Xe $4 \mathrm{~d}$-resonance at $\mathrm{h} \omega \approx 90.5 \mathrm{eV}$. The representative points on Fig.(1) associated with the findings of those analyses and experiments are respectively denoted by [CLU/350] and [Xe/4d*]. Indeed, we note that these data fall precisely on the originally estimated flat contour that connects with the point designating the $\mathrm{Xe}(\mathrm{M})$ threshold at $\hbar \omega=5 \mathrm{eV}$. Hence, the results are uniformly consistent with the Ansatz of frequency independence introduced above. 
The $I$ - h $\omega$ plane presented in Fig.(1) is consequently divided by these experimentally anchored contours into two separate interaction zones, the green quadrant standing at the upper right corresponding to the dynamically enhanced coupling strength, and its contrasting complement, the red zone associated with conventionally described and generally weaker interactions.

The location of the vertical boundary erected at $\mathrm{h} \omega \sim 5 \mathrm{eV}$ in Fig.(1), however, requires further consideration. The phase-dependent enhancement of the interaction can occur only if the period of the driven electron motions is sufficiently short [35,36,42] in comparison with the electronic dephasing time $\tau_{\mathrm{e}}$ associated with the electron-electron interactions in the material. In order to estimate this relaxation time, the L-shell Auger widths of atomic inner-shell vacancies, as noted above, have been successfully used as an indicator [42] of this condition. With this criterion, Xe is a particularly favorable case, since the $\mathrm{L}_{1}$-subshell Auger width of Xe sits at a strong local minimum of $\sim 2-3 \mathrm{eV}$ for atomic numbers in the $50 \leq \mathrm{Z} \leq 60$ range [42, 88-96]. In contrast, the greatest $\mathrm{L}_{1}$ Auger width known is for uranium [96] at $~ 124 \mathrm{eV}$. This datum is indicated on Fig.(1) by $\left[\mathrm{U}\left(\mathrm{L}_{1}\right)\right]$ on the abscissa, the Quantum Energy-axis. It is accordingly expected that the lower quantum energy $\hbar \omega$ boundary of the anomalous region, based on the known range of Auger widths, will depend on the material, particularly in the $5 \mathrm{eV} \leq \hbar \omega \leq 124 \mathrm{eV}$ interval. Hence, in order to define a zone of anomalous interaction that is fully universal, we require the condition $\hbar \omega>>124 \mathrm{eV}$ and set $\hbar \omega \cong 10^{3}$ $\mathrm{eV}$ as the lower bound, a value that ensures the observance of this requirement. This step defines the universal region for enhanced coupling illustrated in Fig.(3) that is designated as the Fundamental Nonlinear Domain.

\begin{tabular}{|c|c|c|c|c|}
\hline Transition & Vacancy & $\begin{array}{c}\text { Emission } \\
\text { Wavelength }(\AA)\end{array}$ & $\begin{array}{c}248 \mathrm{~nm} \\
\text { Threshold } \\
\text { Intensity }\left(\mathrm{W} / \mathrm{cm}^{2}\right)\end{array}$ & Reference \\
\hline $\mathrm{Kr}(\mathrm{L}) 3 \mathrm{~s} \rightarrow 2 \mathrm{p}$ & $2 \mathrm{p}$ & $5.0-7.5$ & $\sim 7 \times 10^{15}$ & 26,85 \\
\hline $\mathrm{Xe}(\mathrm{M}) 4 \mathrm{f} \rightarrow 3 \mathrm{~d}$ & $3 \mathrm{~d}$ & $10-15$ & $\sim 5 \times 10^{15}$ & 37,50 \\
\hline $\mathrm{Xe}(\mathrm{L}) 3 \mathrm{~d} \rightarrow 2 \mathrm{p}$ & $2 \mathrm{p}$ & $2.60-2.95$ & $\sim 2 \times 10^{17}$ & 63 \\
\hline $\mathrm{Xe}(\mathrm{L}) 3 \mathrm{~d} \rightarrow 2 \mathrm{p}$ & $2 \mathrm{~s} 2 \mathrm{p}$ & $2.62-2.84$ & $\sim 8 \times 10^{18}$ & 56 \\
\hline
\end{tabular}

Table I: Experimentally established threshold intensities for $248 \mathrm{~nm}$ irradiation of $\mathrm{Kr}$ and Xe clusters for $\mathrm{Kr}(\mathrm{L}), \mathrm{Xe}(\mathrm{M})$, and $\mathrm{Xe}(\mathrm{L})$ emission arising respectively from $2 \mathrm{p}, 3 \mathrm{~d}$, and $2 \mathrm{p} / 2 \mathrm{~s} 2 \mathrm{p}$ vacancy configurations.

Key supplementary data are also placed on Fig.(3). They include (a) the line

$$
\frac{\mathrm{e} E}{\mathrm{~m} \omega \mathrm{c}}=1
$$

that defines the peak electric field $E$ for the onset of relativistic motions for elections with mass $\mathrm{m}$ and charge e driven at angular frequency $\omega$, (b) the vacuum pair creation limit, the upper value 
bounding the achievable intensity that is associated with ultrarelativistic electron/positron cascades [97], (c) the intensity $\mathrm{I} \cong 4.6 \times 10^{29} \mathrm{~W} / \mathrm{cm}^{2}$ corresponding to the Schwinger/Heisenberg limit [98,99], and (d) the point characterizing the intensity of saturated amplification with the $\mathrm{Xe}(\mathrm{L})$ hollow atom system $[38,39,61-66]$.

The creation of X-ray channels in solids offers the possibility to produce intensities $>10^{27}$ $\mathrm{W} / \mathrm{cm}^{2}$ in high aspect ratio geometries [100,101] and penetrate into the zone shown in Fig.(3) estimated to produce direct coupling to nuclei. These interactions would represent the $\mathrm{x}$-ray analogue of the indirectly induced nuclear reactions generated at optical wavelengths [102-106]. The intensity associated with threshold of channeled propagation of $\mathrm{Xe}(\mathrm{L})$ radiation in solid $\mathrm{U}$ at $\sim 10^{26} \mathrm{~W} / \mathrm{cm}^{2}$ is indicated in Fig.(3) with the green region above it commencing at $\sim 10^{27} \mathrm{~W} / \mathrm{cm}^{2}$ illustrating the entrance into the region associated with the direct induction of nuclear interactions and excitations. Also shown in Fig.(3), as a purple shaded zone in the lower left, is the region associated with the experimental study demonstrating the anomalously enhanced interaction that is discussed in sections 2.2.1 and 2.2.2 below. The analysis indicates that the overall enhancement in the coupling strength for the nonlinear amplitude considered corresponds to a factor of $\sim 3 \times 10^{12}$. 


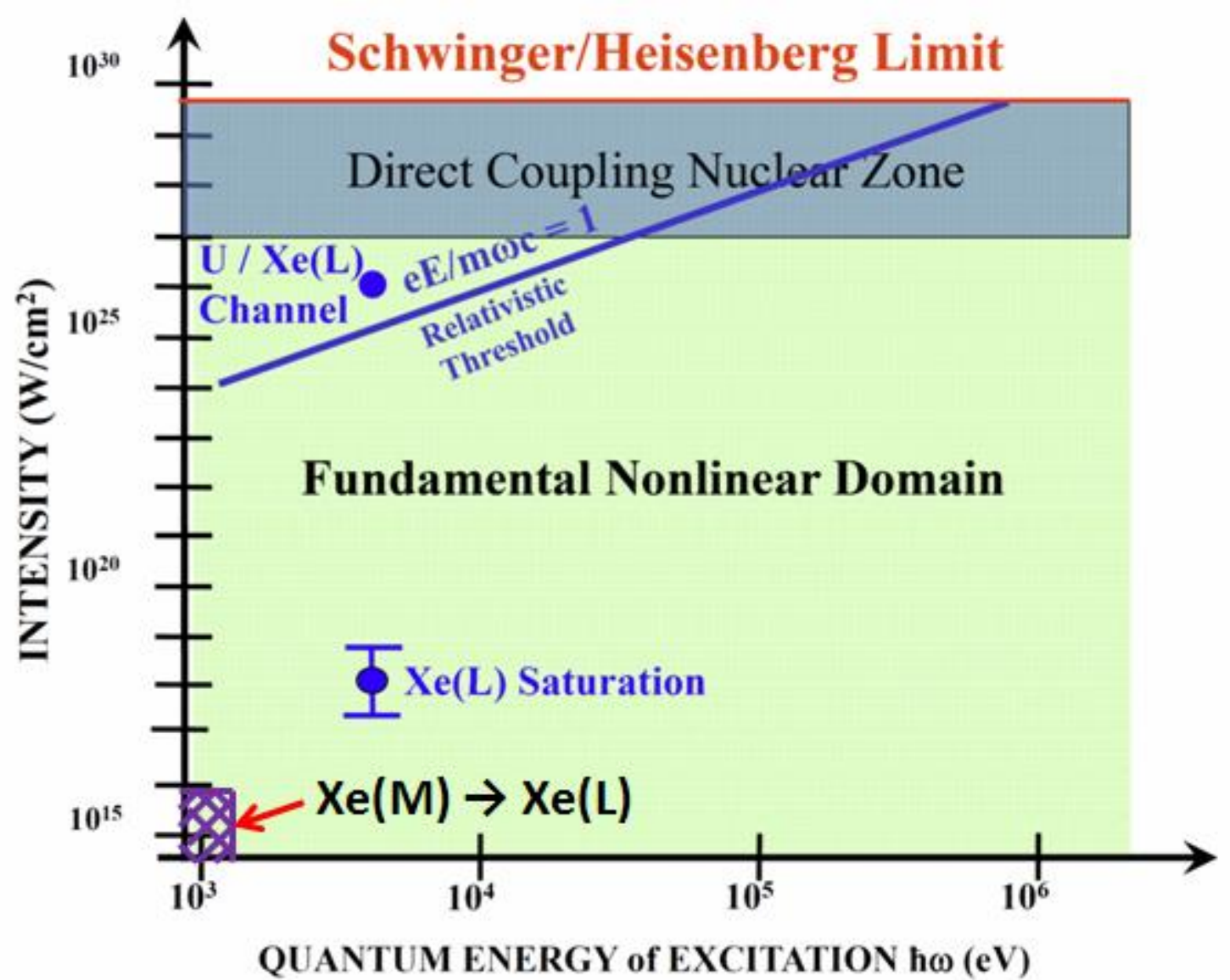

Fig. (3): Universal Fundamental Nonlinear Domain of interactions. The upper intensity limit is taken as the Schwinger/Heisenberg limit $[98,99]$ at $\mathrm{I}=4.6 \times 10^{29} \mathrm{~W} / \mathrm{cm}^{2}$. The zone estimated for direct coupling to nuclei is shown extending upward from $\sim 10^{27} \mathrm{~W} / \mathrm{cm}^{2}$. The contour associated with the onset of relativistic motion of an electron, given by $\mathrm{eE} / \mathrm{m} \omega \mathrm{c}=1$, is shown for reference. The datum associated with saturation of the $\mathrm{Xe}(\mathrm{L})$ hollow atom system $[38,39,61-66]$ is placed at $\hbar \omega \approx 4.5 \mathrm{keV}$ is seen to fall well within the nonlinear domain. The intensity associated with the threshold of channeled propagation of $\mathrm{Xe}(\mathrm{L})$ radiation in $\mathrm{U}$ established by the requirement of a critical power in a channel whose characteristic diameter is $\sim 100 \AA$ at $\mathrm{I} \sim 10^{26} \mathrm{~W} / \mathrm{cm}^{2}$ is shown [102]. The purple shaded zone, designed as $\mathrm{Xe}(\mathrm{M}) \rightarrow \mathrm{Xe}(\mathrm{L})$ located in the lower left, is associated with experiment described in sections 2.2.1 and 2.2.2 that illustrates the new aspects of the enhanced coupling characteristic of the Fundamental Nonlinear Domain in the x-ray region. In comparison with the conventional coupling, the strength of the interaction was augmented by a factor of $\sim 3 \times 10^{12}$. 


\subsection{Experimental Confirmation of Fundamental Nonlinear Domain with Observation of Enhanced Nonlinear Coupling in the X-Ray Range}

\subsubsection{Experimental Evidence}

Experimental studies at high intensities in the kiloelectronvolt x-ray range are beginning to demonstrate the new features of the enhanced coupling predicted to be characteristic of the Fundamental Nonlinear Domain defined in section 2.1 above and illustrated in Fig.(3). In parallel with the earliest detection of nonlinear excitation at visible wavelengths [107], a two-photon amplitude with $\hbar \omega \cong 1.8 \mathrm{eV}$, the first process observed in the new zone of interaction is a corresponding multiphoton excitation that is boosted into the $\mathrm{keV}$ spectral region. Specifically, it is the observed production of $\mathrm{Xe} 2 \mathrm{p}$ vacancies in Xe atoms undergoing irradiation by intense

$\left(\sim 7 \times 10^{15} \mathrm{~W} / \mathrm{cm}^{2}\right) \mathrm{Xe}(\mathrm{M})$ emission at $\hbar \omega \sim 1 \mathrm{keV}$, a process that minimally requires a 5-photon amplitude on energetic grounds. Accordingly, the net reaction is the absorption of $\mathrm{Xe}(\mathrm{M})$ radiation $\gamma_{M}$ at $\hbar \omega \cong 1 \mathrm{keV}$ by $\mathrm{Xe}$ atoms with the liberation of a $2 \mathrm{p}$ electron that is signaled by the subsequent emission of a $\mathrm{Xe}(\mathrm{L})$ quantum on a $3 \mathrm{~d} \rightarrow 2 \mathrm{p}$ transition yielding the detection of photons $\gamma_{\mathrm{L}}$ at $\hbar \omega \approx 4.5$ $\mathrm{keV}$. Hence, $\gamma_{\mathrm{M}}$ is converted to $\gamma_{\mathrm{L}}$ through the multiphoton amplitude

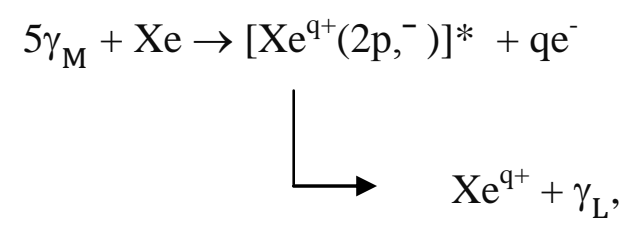

in which the charge state $\mathrm{q}$ represented by the $\mathrm{Xe}^{\mathrm{q}+}$ ion accounts for the level of ionization generated in the complex nonlinear mechanism. The overall reaction represented by Eqs.(5a) and 5(b), that yields the $\mathrm{Xe}^{\mathrm{q}+}+\mathrm{qe}^{-}+\gamma_{\mathrm{L}}$ exit channel, can be properly considered as the $\mathrm{x}$-ray analogue of the previously observed [50] nonlinear excitation of $\mathrm{Xe}(\mathrm{M})$ emission generated by intense $248 \mathrm{~nm}$ irradiation, the mechanism represented by reaction 6(a) that produces the corresponding parallel exit channel $\mathrm{Xe}_{\mathrm{M}}^{+}+\mathrm{q}_{\mathrm{M}} \mathrm{e}^{-}+\gamma_{\mathrm{M}}$. In direct analogy with reactions (5a) and (5b), this process also involves complex multi-electron dynamics with the collateral production of a substantial level of ionization [50].

The initial evidence for the nonlinear amplitude given by Eq.(5) was obtained from simultaneously recorded single-pulse x-ray pinhole camera images that showed remarkably common spatial morphologies of the $\mathrm{Xe}(\mathrm{M})$ and $\mathrm{Xe}(\mathrm{L})$ emissions produced from Xe clusters in a self-trapped $248 \mathrm{~nm}$ channel [26]. These observational data were supplemented by simultaneously recorded Xe(L) spectra and corresponding spatially resolved $\mathrm{Xe}(\mathrm{L})$ longitudinal (Z-axis) emission profiles [38,39] that mapped the distribution of radiation from the zone of excitation. Overall, the $\mathrm{Xe}(\mathrm{L})$ emission $\gamma_{L}$ observed was attributed to the radiative cascade involving the pair of reactions 


$$
\begin{aligned}
& \mathrm{n} \gamma_{248}+\mathrm{Xe} \rightarrow \mathrm{Xe}^{\mathrm{q}_{\mathrm{M}}^{+}}+\mathrm{q}_{\mathrm{M}} \mathrm{e}^{-}+\gamma_{\mathrm{M}} \\
& 5 \gamma_{\mathrm{M}}+\mathrm{Xe} \stackrel{\boldsymbol{\sigma}_{5}}{\rightarrow} \mathrm{Xe}^{\mathrm{q}_{\mathrm{L}}^{+}}+\mathrm{qe}_{\mathrm{L}}^{-}+\gamma_{\mathrm{L}}
\end{aligned}
$$

in which the $\mathrm{Xe}(\mathrm{M})$ radiation $\gamma_{\mathrm{M}}$ produced through reaction 6(a) is absorbed by neutral Xe atoms in the immediately neighboring spatial region of the channel [26] that is unaffected directly by the incident $248 \mathrm{~nm}$ wave. The key process generating the $\gamma_{\mathrm{L}}$ emission is reaction (6b) with the effective nonlinear cross section $\sigma_{5}$. Since the x-ray camera is calibrated, the quantitative analysis of the $\mathrm{Xe}(\mathrm{M})$ and $\mathrm{Xe}(\mathrm{L})$ signal strengths experimentally measured showed that $\sigma_{5} \sim 2 \times 10^{-21} \mathrm{~cm}^{2}$ at the ambient $\mathrm{Xe}(\mathrm{M})$ intensity [26] associated with the propagation. The specific experimental conditions and results of this analysis are given below in section 2.2.2.

The experimental configuration utilized for these observations is illustrated schematically in Fig.(4). The key instruments are the multiple pinhole x-ray camera, that simultaneously records images the $\mathrm{Xe}(\mathrm{M})$ and $\mathrm{Xe}(\mathrm{L})$ emissions, and the von Hámos X-ray spectrometer that transversely records the $\mathrm{Xe}(\mathrm{L})$ spectrum with spatial resolution along the axial $(\mathrm{Z})$ direction. Initially, we consider the X-ray pinhole camera images shown in Fig.(5) that present the spatial morphologies of the $\mathrm{Xe}(\mathrm{M})$ and $\mathrm{Xe}(\mathrm{L})$ emissions generated from self-trapped plasma channels [12-26] produced by a femtosecond $248 \mathrm{~nm}$ pulse in the Xe cluster target [26]. Prominently exhibited by the $\mathrm{Xe}(\mathrm{M})$ and $\mathrm{Xe}(\mathrm{L})$ images are corresponding spatially matching extended emission zones in the $\mathrm{Z}>-1 \mathrm{~mm}$ longitudinal region. Significantly, the transverse spatial width of the observed $\mathrm{Xe}(\mathrm{L})$ emission is $\delta_{\mathrm{L}} \cong$ $250 \mu \mathrm{m}$. Since the threshold intensity for $\mathrm{Xe}(\mathrm{L})$ production with $248 \mathrm{~nm}$ excitation is known [63] to be $\mathrm{I}_{\mathrm{L}} \sim 2 \times 10^{17} \mathrm{~W} / \mathrm{cm}^{2}$, as given in Table I, a feature of this transverse size would require a minimum $248 \mathrm{~nm}$ power of

$$
\mathrm{P}_{248} \cong \mathrm{I}_{\mathrm{L}} \delta_{\mathrm{L}}^{2} \cong 125 \mathrm{TW}
$$

if direct excitation by the $248 \mathrm{~nm}$ wave were to generate the observed $\mathrm{Xe}(\mathrm{L})$ emission. However, since these experiments were conducted with a $248 \mathrm{~nm}$ source unquestionably incapable of delivering a power greater than 3-5 TW, as described below in section 3, the previously established mechanism producing $\mathrm{Xe}(\mathrm{L})$ hollow atom emission with $248 \mathrm{~nm}$ excitation [34,38] is completely ruled out as the source of the excitation leading to the broadly extended emission seen in the $\mathrm{Z}>-1 \mathrm{~mm}$ region. Conversely, since it is experimentally established [26] that $\mathrm{Xe}(\mathrm{M})$ radiation produced by the channels saturates the Xe absorption under the conditions of this experiment and thereby readily propagates for extended lengths, the nearly perfect spatial overlap of the $\mathrm{Xe}(\mathrm{M})$ and $\mathrm{Xe}(\mathrm{L})$ emissions evidences the strong direct coupling that occurs through the reactions represented by Eq.(5) that convert $\mathrm{Xe}(\mathrm{M})$ radiation to $\mathrm{Xe}(\mathrm{L})$.

The key data from three experimental files, documented in Figs.(5), (6), and (7) following, illustrate the properties of the $\mathrm{Xe}(\mathrm{M})$ to $\mathrm{Xe}(\mathrm{L})$ conversion represented by the mutual radiative coupling specifically expressed by Eqs.(5a) and (5b). The axial (Z) traces pictured in Fig.(6) of the isometric views of the $\mathrm{Xe}(\mathrm{M})$ and $\mathrm{Xe}(\mathrm{L})$ spectra shown in Fig.(5) illustrate a refined visualization of these longitudinal profiles. Three characteristics are prominent in Figs.(5) and (6). First, the main $\mathrm{Xe}(\mathrm{M})$ and $\mathrm{Xe}(\mathrm{L})$ signals respectively shown in Figs.(5a) and (5b) for $\mathrm{Z}<-1 \mathrm{~mm}$ exhibit nearly identical spatial contours with closely matching transverse widths of $\sim 250 \mu \mathrm{m}$. Second, the 
corresponding $\mathrm{Xe}(\mathrm{L})$ and the $\mathrm{Xe}(\mathrm{M})$ extensions in the $\mathrm{Z}>-1 \mathrm{~mm}$ region in these two panels both have lengths $\sim 500 \mu \mathrm{m}$. Hence, the correspondence of the $\mathrm{Xe}(\mathrm{M})$ and $\mathrm{Xe}(\mathrm{L})$ spatial morphologies in Fig.(5), both laterally and longitudinally, speak to a direct physical coupling as the origin of the $\mathrm{Xe}(\mathrm{L})$ emission. This is the expected spatial signature associated with reaction (6b).

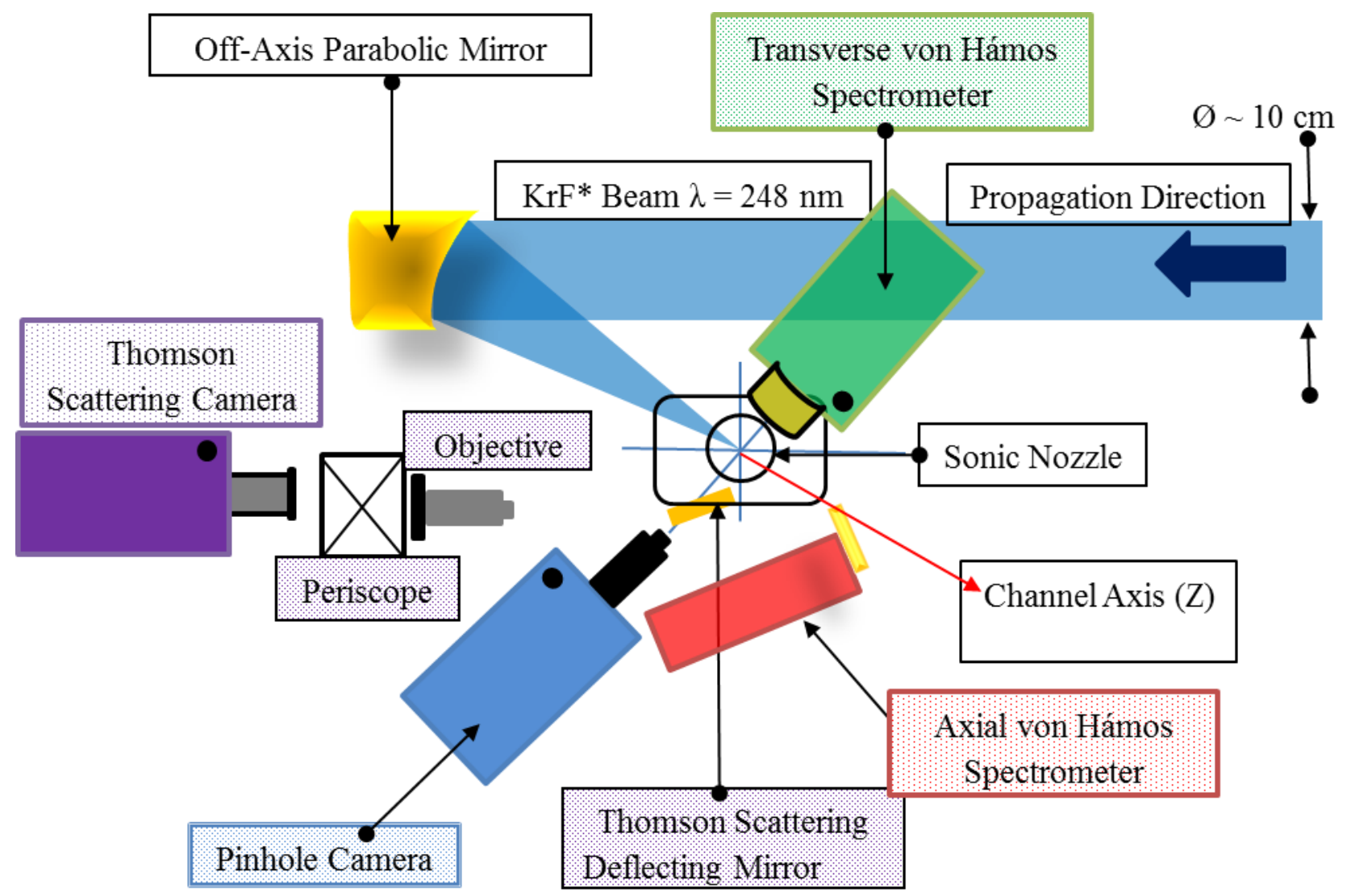

Fig.(4): The arrangement of the key diagnostic components is illustrated showing the pinhole x-ray camera utilized to obtain simultaneously the images of the $\mathrm{Xe}(\mathrm{M})$ and $\mathrm{Xe}(\mathrm{L})$ emissions represented in Fig.(5) and the transverse von Hámos spectrometer enabling the recording of a spatially resolved $\mathrm{Xe}(\mathrm{L})$ spectrum along the axis of the channel with a spectral resolution of $\sim 3 \mathrm{eV}$ and a spatial resolution of $\sim 50 \mu \mathrm{m}$. The Thomson imaging system used for measurements of the electron density is shown along with axial von Hámos spectrometer. The incident $248 \mathrm{~nm}$ beam with a pulse rate of $\sim 0.1 \mathrm{~Hz}$ and diameter of $\sim 10 \mathrm{~cm}$ enters from the right and is focused by the off-axis parabolic mirror to the position of the Xe cluster target. The circular orifice of the $2.65 \mathrm{~mm}$ diameter sonic nozzle that furnishes the $(\mathrm{Xe})_{\mathrm{n}}$ target with upward vertically directed flow is indicated. The single-pulse data from the two cameras are collected under computer control along with the $248 \mathrm{~nm}$ pulse energy, the Xe nozzle plenum pressure, and the Xe gas temperature. Additional experimental details are available in Refs.[26] and [85]. 
(a)
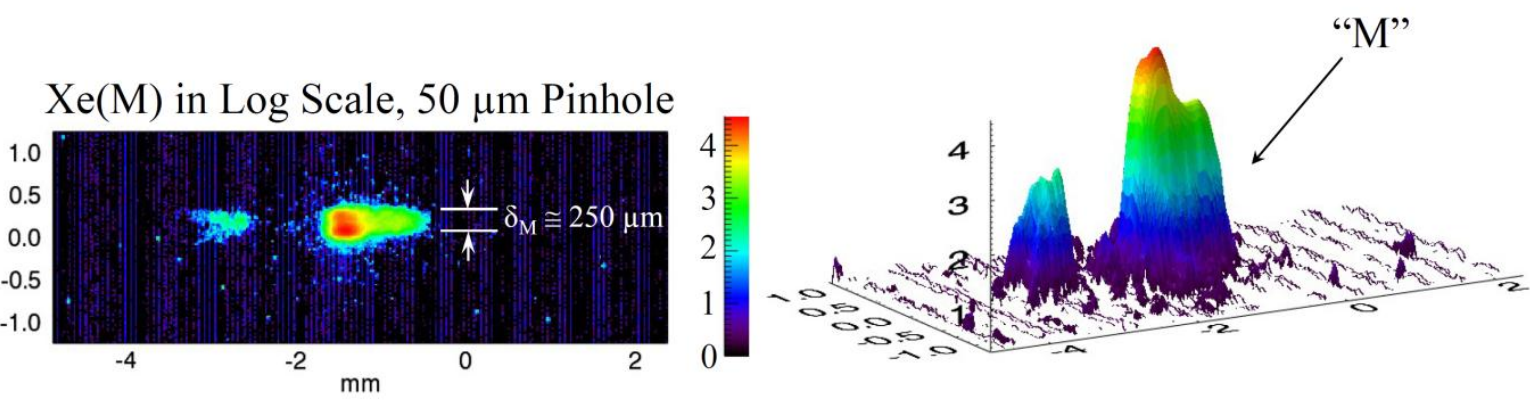

248 nm Propagation

(b)
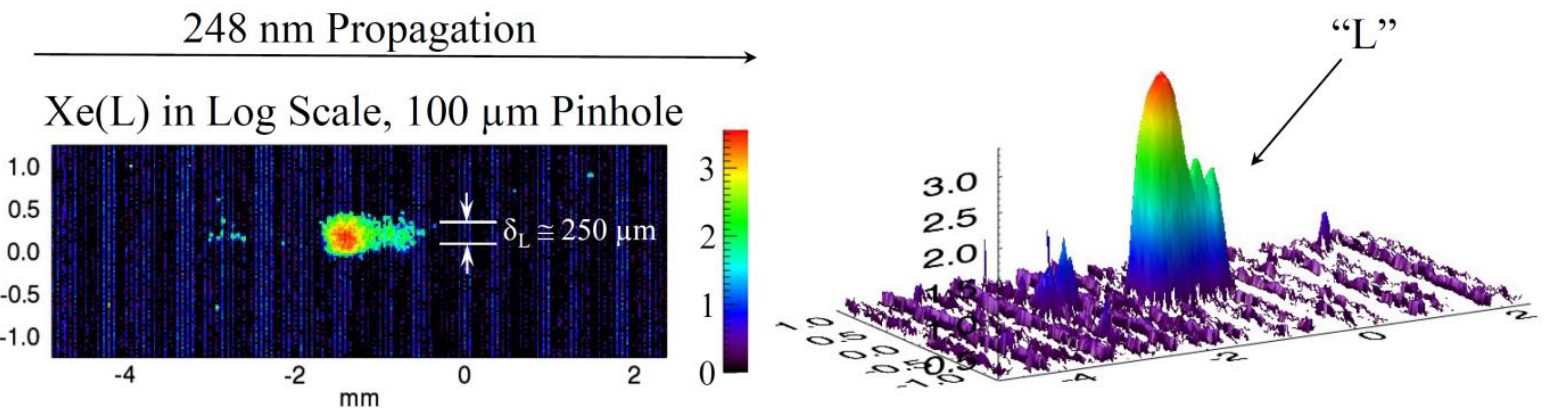

$\mathrm{Xe}(\mathrm{L})$ in Log Scale, $100 \mu \mathrm{m}$ Pinhole

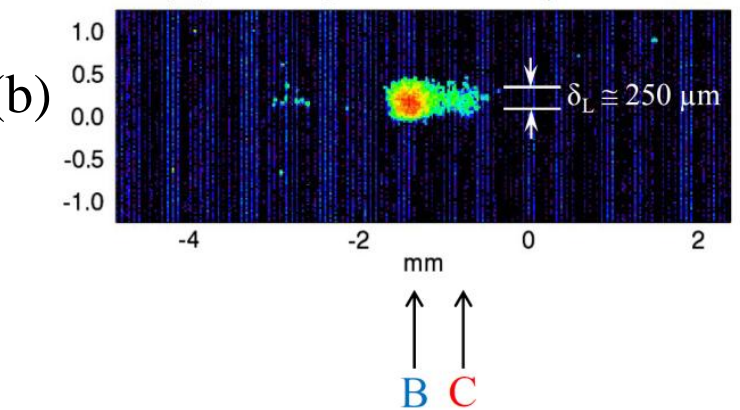

Fig.(5): Simultaneous single-pulse $\mathrm{Xe}(\mathrm{M})$ and $\mathrm{Xe}(\mathrm{L}) \mathrm{x}$-ray pinhole camera spatial morphologies; file \#144/2 February 2012. Experimental parameters: pulse energy $260 \mathrm{~mJ}$, Xe plenum pressure $162 \mathrm{psi}$, and Xe plenum temperature $296 \mathrm{~K}$. The direction of propagation of the $248 \mathrm{~nm}$ pulse is left to right. The images shown are: $\mathrm{Xe}(\mathrm{M})$ emission, panel (a), and the corresponding $\mathrm{Xe}(\mathrm{L})$ emission, panel (b); transverse images with log scale intensity (left) with associated isometric views (right). Extended zones of emission of the $\mathrm{Xe}(\mathrm{M})$ and $\mathrm{Xe}(\mathrm{L})$ signals occur in the range $\mathrm{Z}>-1 \mathrm{~mm}$ with corresponding transverse widths of $\delta_{\mathrm{M}} \cong 250 \mu \mathrm{m}$ and $\delta_{\mathrm{L}} \cong 250 \mu \mathrm{m}$, dimensions that are closely matching. The isometric renderings detail the corresponding structures in the emissions. Significantly, the strongly peaked crests in the "L" pinhole panel (b) in comparison to the matching features in pinhole "M" of panel (a), exhibit the canonical sharpening sign of nonlinearity expected in the $\mathrm{Xe}(\mathrm{M}) \rightarrow \mathrm{Xe}(\mathrm{L})$ amplitude given by the reaction designated by Eq.(6b). Further details of these modulated structures are presented in Fig.(6). 


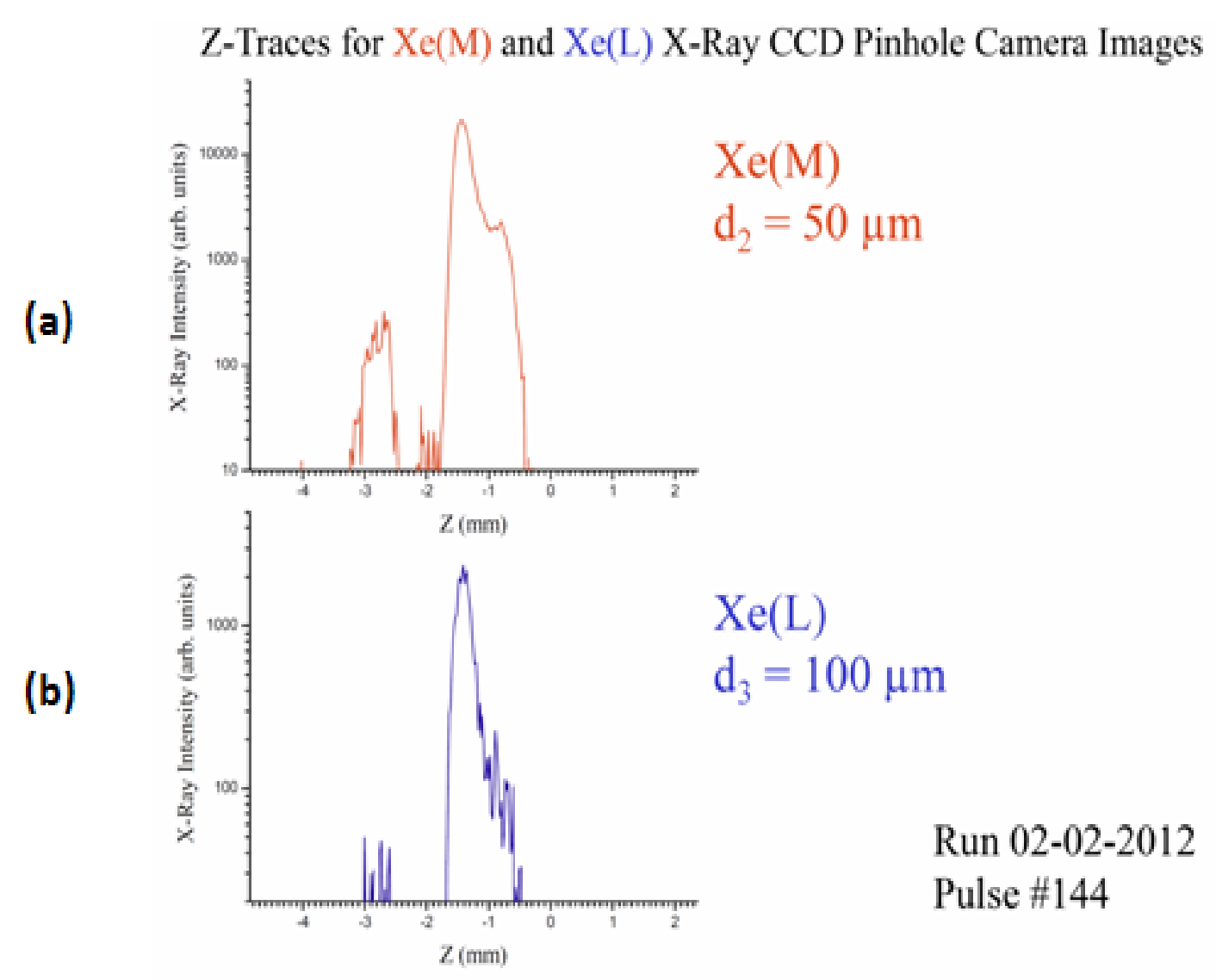

Fig.(6) : Axial (Z) spatial profiles of $\mathrm{Xe}(\mathrm{M})$ and $\mathrm{Xe}(\mathrm{L})$ emissions shown in Fig.(5) for file \#144/2 February 2012. The pinholes in the x-ray camera have diameters of $d_{2}=50 \mu \mathrm{m}$ and $\mathrm{d}_{3}=100 \mu \mathrm{m}$, respectively, for the $\mathrm{Xe}(\mathrm{M})$ and $\mathrm{Xe}(\mathrm{L})$ signals. Accordingly, the spatial resolution of the $\mathrm{Xe}(\mathrm{M})$ distribution is $\sim \mathrm{d}_{3} / \mathrm{d}_{2} \cong 2$ times better than the corresponding $\mathrm{Xe}(\mathrm{L})$ profile. (a) $\mathrm{Xe}(\mathrm{M})$ longitudinal (Z) profile showing the X-ray signal depicted in Fig.5(a). The strong peak in the emission possesses a structured extension into the region $\mathrm{Z}>-1 \mathrm{~mm}$. (b) The corresponding $\mathrm{Xe}(\mathrm{L})$ longitudinal (Z) profile shown in Fig.5(b) is depicted. In parallel with the $\mathrm{Xe}(\mathrm{M})$ profile, a strong peak is also associated with a structured extension into the $Z>-1 \mathrm{~mm}$ zone. The signals shown in panels (a) and (b) exhibit remarkably similar spatial morphologies that indicate a direct physical coupling. In the extended region $(Z>-1 \mathrm{~mm})$, the $\mathrm{Xe}(\mathrm{L})$ signal exhibits strong modulation with features of $\sim 100 \mu \mathrm{m}$ in width, a characteristic that contrasts significantly with the $\mathrm{Xe}(\mathrm{M})$ signal that shows perceptible, but far weaker modulation, even though the spatial resolution is approximately two-fold greater; the conclusion that the $\mathrm{Xe}(\mathrm{L})$ modulations are considerably sharper and more pronounced than those illustrated by the $\mathrm{Xe}(\mathrm{M})$ counterpart follows. 
(a)
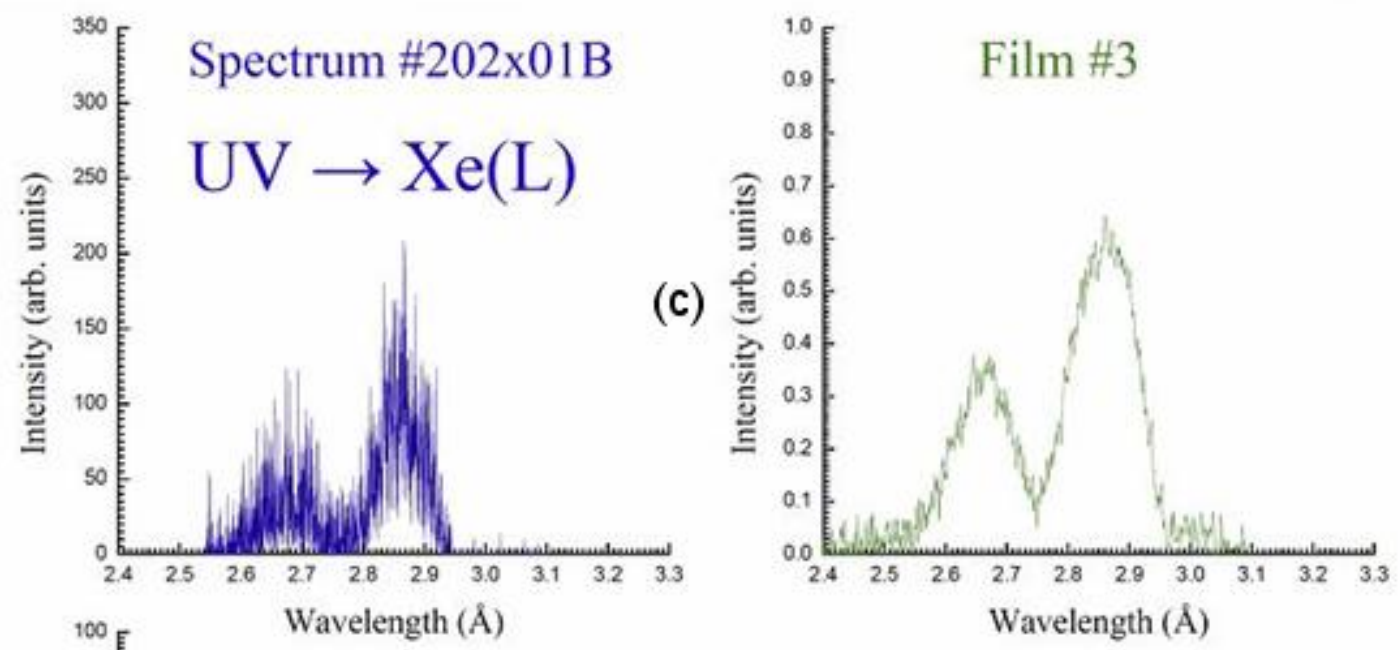

(b)

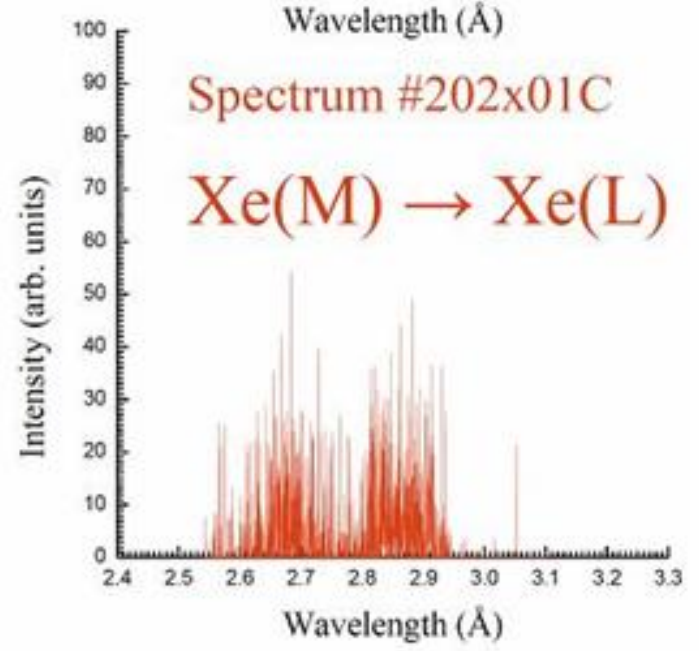

Fig.(7) : Single-pulse $\mathrm{Xe}(\mathrm{L})$ spectra respectively corresponding to locations B and C designated in panel (b) of Fig. (5) from file \#144 (2 February 2012) together with comparative film data presented in panel (c) (film \#3) showing the canonical $\mathrm{Xe}(\mathrm{L})$ hollow atom spontaneous emission spectrum produced from Xe clusters with $248 \mathrm{~nm}$ excitation whose properties are known from earlier studies $[38,61,62]$. (a) $\mathrm{Xe}(\mathrm{L})$ spectrum recorded at point B in Fig.5(b). The emission occurs in the $\mathrm{Xe}(\mathrm{L})$ spectral region for which $3 \mathrm{~d} \rightarrow 2 \mathrm{p}$ transitions in $\mathrm{Xe}^{\mathrm{q}+}$ ions are the known source [108]. This spectrum is a manifestly good match to the canonical $\mathrm{Xe}(\mathrm{L})$ hollow atom spectrum shown in panel (c) from film \#3, (b) $\mathrm{Xe}(\mathrm{L})$ spectrum recorded at location C specified in Fig.(5b) that is attributed to nonlinear excitation by ambient $\mathrm{Xe}(\mathrm{M})$ radiation through the nonlinear reaction given by Eq.(6b). The two spectral lobes match well with the corresponding features associated with the $\mathrm{Xe}(\mathrm{L})$ hollow atom spectra illustrated in both panels (a) and (c). (c) Previously established canonical Xe(L) hollow atom spontaneous emission spectrum excited by $248 \mathrm{~nm}$ radiation [38].

The third important observation is shown in Fig.(6) in the $Z>-1 \mathrm{~mm}$ zone, where the strong sharp modulations seen in the $\mathrm{Xe}(\mathrm{L})$ signal are very greatly reduced in the corresponding $\mathrm{Xe}(\mathrm{M})$ recording. This observation is the canonical behavior of a high-order nonlinear amplitude of the kind given by Eq.(6b) in which a fifth-order mechanism produces the $\mathrm{Xe}(\mathrm{M}) \rightarrow \mathrm{Xe}(\mathrm{L})$ conversion; specifically small variations in the ambient $\mathrm{Xe}(\mathrm{M})$ intensity, peak " $\mathrm{M}$ " in Fig.(5a), generate 
proportionally much larger fluctuations in the $\mathrm{Xe}(\mathrm{L})$ excitation corresponding to the peak " $\mathrm{L}$ " in Fig.(5b).

The $\mathrm{Xe}(\mathrm{L})$ spectra illustrated in Fig.(7) reveal additional important characteristics of the emission. The single-pulse spectrum shown in panel (a) corresponds very well to the canonical $\mathrm{Xe}(\mathrm{L})$ $3 \mathrm{~d} \rightarrow 2 \mathrm{p}$ hollow atom spectrum produced with $248 \mathrm{~nm}$ pulses from Xe clusters [38] presented by the recording from film \#3 in panel (c); the two spectra are essentially identical. Likewise, the $\mathrm{Xe}(\mathrm{L})$ spectrum illustrated in panel (b), that is attributed to the nonlinear $\mathrm{Xe}(\mathrm{M})$ to $\mathrm{Xe}(\mathrm{L})$ conversion expressed by Eq.(6b), exhibits good correspondence with the two $\mathrm{Xe}(\mathrm{L})$ spectra shown in panels (a) and (c). Hence, all three spectral profiles stand as equivalent. The conclusion that all spectra represent $\mathrm{Xe}(\mathrm{L}) 3 \mathrm{~d} \rightarrow 2 \mathrm{p}$ hollow atom emissions follows.

\subsubsection{Analysis of the $\mathrm{Xe}(\mathrm{M}) \rightarrow \mathrm{Xe}(\mathrm{L}) 5 \gamma$ Cross Section $\sigma_{5}$}

\subsubsection{Formulation of Estimated Cross Section}

We presently turn to an analysis of the nonlinear amplitude expressed by Eq.(6b). The process is essentially a 5-photon ionization of the 2 p-shell with $\mathrm{Xe}(\mathrm{M})$ radiation at $\hbar \omega_{\mathrm{M}} \sim 1 \mathrm{keV}$ that subsequently yields $\mathrm{Xe}(\mathrm{L})$ quanta at $\hbar \omega_{\mathrm{L}} \cong 4.5 \mathrm{keV}$. In order to evaluate approximately the scale of this $5 \gamma$ cross section $\sigma_{5}$, we can represent the magnitude initially as the conventional product of two terms given by

$$
\sigma_{5} \cong \sigma_{\gamma}\left(\frac{\mu \mathrm{E}}{\Delta \hbar \omega}\right)^{2 n} .
$$

We will subsequently introduce a radical modification into this conventional statement in order to account for the dynamically enhanced coupling that arises in the nonlinear amplitude expressed by Eq.(6b). In Eq.(8), $\sigma_{\gamma}$ denotes a characteristic cross section for the reaction which is taken for this estimate as the measured peak value for the linear photoionization [109] of Xe atoms; hence, we put $\sigma_{\gamma} \cong 5 \times 10^{-18} \mathrm{~cm}^{2}$. The energy denominator $\Delta \hbar \omega$ in Eq.(8), based on the characteristic energies of the xenon atom [109], is assigned the value of $\sim 10^{3} \mathrm{eV}$, a magnitude that falls near the peak of the $\mathrm{Xe}(\mathrm{M})$ range.

On the basis of the signal strengths observed with the calibrated x-ray cameras [26], the measured value of $\sigma_{5}$ was found to be

$$
\sigma_{5 \mathrm{~m}}=2 \times 10^{-21} \mathrm{~cm}^{2} .
$$

Accordingly, we calculate below the estimated value $\sigma_{5}$ from Eq.(8) for $\mathrm{n}=5$ and compare this computed magnitude with the observed value $\sigma_{5 \mathrm{~m}}$.

The value of the electric field strength $\mathrm{E}$ in Eq.(8) representing the ambient $\mathrm{Xe}(\mathrm{M})$ radiation is experimentally established by studies [26] of $248 \mathrm{~nm}$ self-trapped channel production in a Xe cluster medium. The $\mathrm{Xe}(\mathrm{M})$ intensity found in that work was $\sim 7.5 \times 10^{15} \mathrm{~W} / \mathrm{cm}^{2}$, a value that places the field at $\mathrm{E} \cong 2.4 \times 10^{9} \mathrm{~V} / \mathrm{cm}$, a magnitude that is roughly equivalent to one half of an atomic unit $\left(\mathrm{e} / a_{0}^{2}\right)$. 


\subsubsection{Fundamental Ansatz}

In order to perform the computation of the value of $\sigma_{5}$ given by Eq.(8), it remains to estimate the effective dipole moment $\mu$ of the electronic motion induced by the electric field $\mathrm{E}$ associated with the ambient $\mathrm{Xe}(\mathrm{M}) \sim 1 \mathrm{keV}$ radiation. At this point, we introduce a fundamental Ansatz that represents a gross departure from standard perturbative treatments that are associated with expansions involving terms of the kind given by Eq.(8). Accordingly, we write the dipole moment as

$$
\mu=\operatorname{Zex}
$$

with $\mathrm{Z}$ designating the number of coherently driven electrons, e the electronic charge, and $\mathrm{x}$ the radial scale size of active electrons. With $\alpha$ designating the fine structure constant, this modification of the dipole moment imports the replacement $\alpha=>Z^{2} \alpha$ for the basic coupling constant of the system. This key step is physically equivalent to the selection in the state manifold of the Z-fold multiply excited $\underline{\text { states }}[27,28,30,47,110]$ as the dominant contribution to the amplitude. Since it is known from an abundance of studies examining the mechanisms of photoionization of $\mathrm{Xe}$ that the 18 electrons comprising the three relatively weakly bound outer atomic shells $\left(5 \mathrm{p}^{6} 5 \mathrm{~s}^{2} 4 \mathrm{~d}^{10}\right)$ are strongly coupled $[111,112]$ and act as a single "super-shell", we put $Z=18$.

The value of the dipole scale length $\mathrm{x}$ can be naturally associated with the orbital size of the $4 \mathrm{~d}$ orbital, since the $4 \mathrm{~d}$ electrons are the dominant members of the "super-shell". The spatial properties of the $\mathrm{Xe} 4 \mathrm{~d}$-orbital [113] are shown in Fig.(8), a result that was computed from consideration of electron-impact ionization. On the basis of these data, we assign the value

$$
\mathrm{x} \cong 2 \mathrm{a}_{\mathrm{o}}=1.06 \times 10^{-8} \mathrm{~cm} .
$$

It is now possible to evaluate the estimated magnitude of $\sigma_{5}$ from Eq.(8) and a summary of this computation is presented in Table II. The estimated value of $\sigma_{5}$ detailed in Table II falls in very good correspondence with the measured magnitude stated in Eq.(9). Furthermore, without the incorporation of the Ansatz giving $Z=18$, a gross disagreement of $\sim 10^{12}$ in the magnitudes would have been found. The values of both $\sigma_{5}$ and $\sigma_{5 \mathrm{~m}}$ can furthermore be compared to an earlier general study aimed at the determination of limiting cross sections for high-order multiphoton coupling [46]. The result found in those computations is illustrated in Fig.(9). We observe that the values for $\sigma_{5}$ correspond quite well with the magnitude of the effective nonlinear cross section estimated in that work whose goal was the determination of an upper-bound. As shown in Fig.(9), the limiting crosssection $\sigma_{\mathrm{m}}=8 \pi \lambda_{0}^{2}$, depends only upon the mass of the electron and is independent of the frequency $\omega$ and any atomic parameters. It is accordingly possible to interpret the abscissa in Fig.(9) as an intensity that is independent of wavelength. We also note that these results fall in good accord with recent findings of Richter [47] concerning experiments that used a quantum energy of $\hbar \omega \sim 100 \mathrm{eV}$

and an intensity of $\sim 10^{15} \mathrm{~W} / \mathrm{cm}^{2}$ in which a similar physical picture is used. In all cases, the organized motion involving $\mathrm{Z} \gg 1$ is necessary to reconcile the experimental observations with a corresponding theoretical value for the amplitude. 


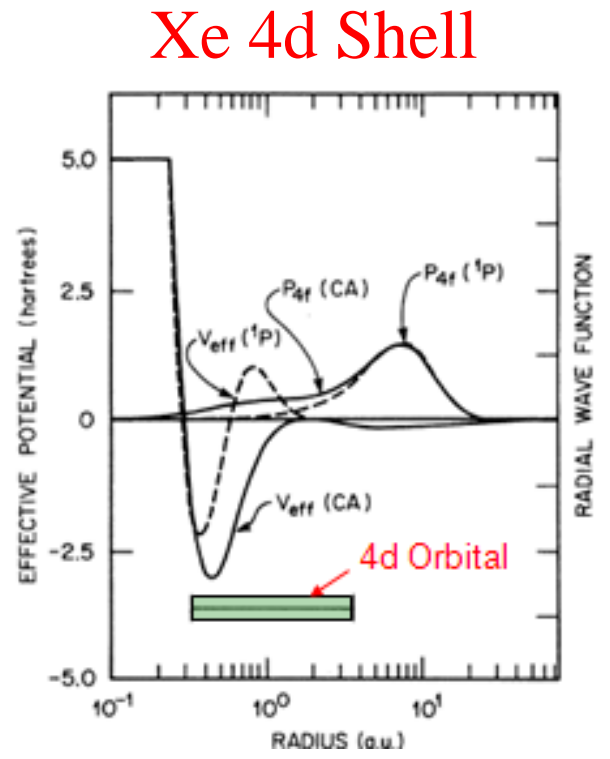

Fig. (8): Plots of the $4 f(\mathrm{CA})$ (solid curves) and $4 \mathrm{f}\left({ }^{1} \mathrm{p}\right)$ (dotted curves) effective potentials and radial wave functions for the $4 d^{9} 5 s^{2} 5 p^{5} 4 f$ configuration in $\mathrm{Xe}^{+}$. A logarithmic scale is used for the radius. The bar indicates the range of the radii over which the second antinode of the $4 d$ radial wave function (in the ground-state configuration) occurs. The figure is adopted from Ref. [113] and used with permission. 


$$
\begin{aligned}
& \mathrm{Xe}(\mathrm{M}) \rightarrow \mathrm{Xe}(\mathrm{L}) 5 \gamma \text { Estimate } \\
& \sigma_{5 \gamma}=\sigma_{\gamma}\left(\frac{\mu E}{\hbar \Delta \omega}\right)^{2 n} \quad 2 \mathrm{n}=10
\end{aligned}
$$

$\sigma_{\gamma} \cong 5 \times 10^{-18} \mathrm{~cm}^{2} \leftarrow$ Measurement

$\sigma_{5 m} \cong 2 \times 10^{-21} \mathrm{~cm}^{2} \leftarrow$ Measurement

We need: $\quad\left(\frac{\mu E}{\hbar \Delta \omega}\right)^{10} \cong 4 \times 10^{-4} \quad\left(\frac{\mu E}{\hbar \omega}\right) \cong 0.457$

We have: $\mu=\operatorname{ZeX}, Z=18, \quad x \cong 2 a_{0} \cong 1.06 \times 10^{-8} \mathrm{~cm}$ (4d shell)

$$
\frac{\mu}{e} \cong 1.9 \times 10^{-7} \mathrm{~cm} \quad E \cong 2.4 \times 10^{9} \frac{\mathrm{V}}{\mathrm{cm}} \quad \Delta \hbar \omega \cong 10^{3} \mathrm{eV}
$$

Thus: $\quad \frac{\mu E}{\hbar \Delta \omega} \cong \frac{\left(1.9 \times 10^{-7} \mathrm{~cm}\right) \mathrm{e}\left(2.8 \times 10^{9} \mathrm{~V} / \mathrm{cm}\right)}{10^{3} \mathrm{eV}}=0.46$ (estimated)

Conclusion: $\alpha \Rightarrow Z^{2} \alpha \Rightarrow 2.4>1 \quad Z=18$

$0,5=2,1 \times 10-21 \mathrm{~cm}^{2}$

Table II: Summary of the computation of the estimated value of the Nonlinear Cross Section $\sigma_{5}$. The final estimated value is $\sigma_{5} \cong 2.1 \times 10^{-21} \mathrm{~cm}^{2}$ for $\mathrm{Z}=18$. This magnitude is in excellent correspondence with the measured value of $\sigma_{5 \mathrm{~m}} \cong 2 \times 10^{-21} \mathrm{~cm}^{2}$. We note that without the factor of $\mathrm{Z}^{10} \cong 3.6 \times 10^{12}$, the estimated value of $\sigma_{5}$ would be reduced to the nanobarn range, a level that would have been experimentally unobservable by a margin greater than $10^{9}$. 


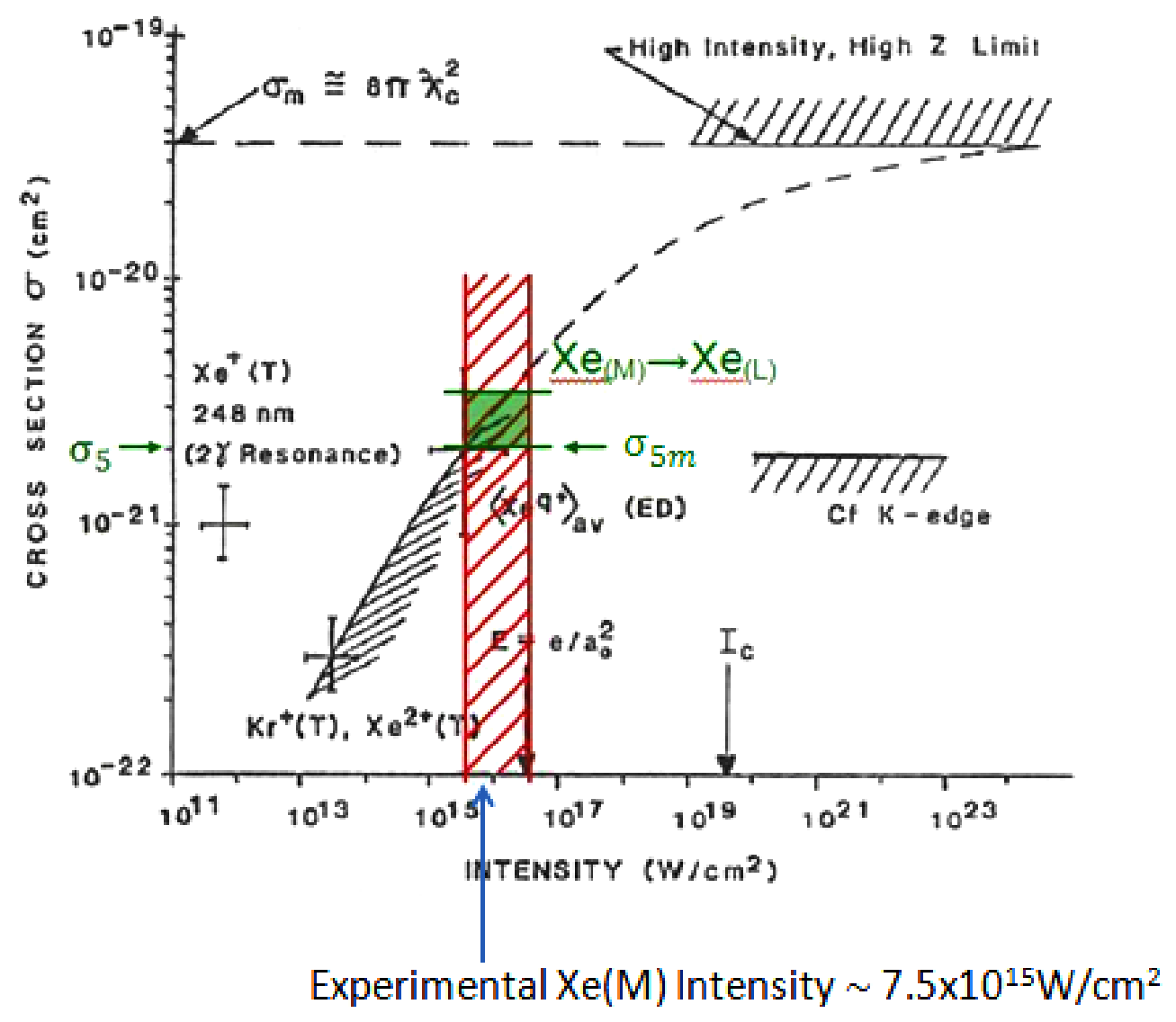

Fig.(9): Plot illustrating measured, estimated, and extrapolated cross sections for total energy deposition versus $248 \mathrm{~nm}$ intensity for subpicosecond irradiation under collision-free conditions as presented originally in Ref.[46]. Data marked with (T) were determined from threshold measurements. The symbol ED refers to total energy deposition. Note the two-photon resonance applying to the $\mathrm{Xe}^{+}$ datum. Ic denotes the Compton Intensity, $\mathrm{E}$ is the ultraviolet electric field strength, and for $\sigma_{\mathrm{m}}$ refer to Ref.[46]. The dashed line --- represents an extrapolation of the data to the value $\sigma_{\mathrm{m}}$ in the high field limit and the value corresponding to total photoabsorption cross section for the $\mathrm{K}$-edge of $\mathrm{Cf}$ is indicated for references in the high intensity region. The locations of $\sigma_{5}$ and $\sigma_{5 \mathrm{~m}}$ are shown at the experimental $\mathrm{Xe}(\mathrm{M})$ intensity of $\sim 7.5 \times 10^{15} \mathrm{~W} / \mathrm{cm}^{2}$. A good overlap with the extrapolated curve originally estimated [46] is seen at the value of $\sim 2 \times 10^{-21} \mathrm{~cm}^{2}$, as indicated by the purple rectangle. Since the limiting cross section $\sigma_{\mathrm{m}} \cong 8 \pi \lambda_{0}^{2}$ is independent of the frequency $\omega$, the abscissa can be generally interpreted to represent validly the intensity of the $\mathrm{Xe}(\mathrm{M}) \mathrm{x}$-rays.

The significance of the nonlinear coupling associated with Eqs.(5) and (6b) is highlighted by the location of the purple $\mathrm{Xe}(\mathrm{M})$ rectangle shown in Fig.(6); it falls essentially at the lower limit of the zone given by $\sim 10^{15} \mathrm{~W} / \mathrm{cm}^{2}$ in Fig.(1). Furthermore, on the basis of the measured threshold [63] of $\sim 2 \times 10^{17} \mathrm{~W} / \mathrm{cm}^{2}$ for the corresponding production of $\mathrm{Xe}(\mathrm{L})$ emission with $248 \mathrm{~nm}$ radiation $\hbar \omega \cong 5$ $\mathrm{eV}$, we see that the threshold value for $2 \mathrm{p}$-shell excitation has decreased by a factor of $\sim 10^{2}$ at the higher quantum energy of $\hbar \omega \sim 1 \mathrm{keV}$. It follows that the circumstances for the production of deep inner-shell vacancies could hardly be more favorable; the minimally required intensity that provides 
strong excitation of the key states of main interest for the production of highly energetic radiation has been strongly lowered. Furthermore, since it is experimentally known [26] that Xe(M) radiation can be efficiently produced $(>20 \%)$, this greatly reduced threshold now enables the consideration of the excitation of correspondingly increased volumes of material with result that x-ray pulse energies in the 1-10 J range can now be practically contemplated, since the technological base of high pulse energy $\mathrm{KrF}^{*}$ system is solidly established. This magnitude for the $\mathrm{x}$-ray energy in a single pulse of femtosecond duration is utterly out of reach by accelerator technology. Although a complete theoretical picture of the complex phenomenon producing the inner-shell vacancies is not in hand, the present experiments combine two conditions in an incomparably new situation; specifically, it is the alliance of (1) a strong radiation field on the order of e/ $a_{0}^{2}$ and (2) a high frequency $\omega$ whose period is less than 5 as. Since a time of $\sim 5$ as is far less than an electron dephasing time in all materials, this situation is well suited for the production of unconventional highly organized electronic motions in all materials. Hence, in forthcoming studies we can anticipate the production of numerous new exceptional forms of energetically excited matter that are analogous to the examples $[32,34]$ provided by $\mathrm{N}_{2}$ and the Xe hollow atom states. And, as explained below in section 4, these favorable developments may possibly be extended to nuclear systems.

\section{Femtosecond UV 248 nm High-Brightness Laser Technology}

\subsection{Introduction}

The data presented in section 2, that enabled the construction of Fig.(1) with the corresponding definition of the boundaries of the Fundamental Nonlinear Domain, were exclusively obtained with the use of Excimer Laser Technology. The basis of this unique role was the ability to satisfy simultaneously a triplet $(\omega, \mathrm{I}, \mathrm{P})$ of conditions stating the minimal values of the frequency $\omega$, intensity $\mathrm{I}$, and the power $\mathrm{P}$ necessary for the two key physical processes involved to be experimentally observed and controllably combined [37]. Specifically, these phenomenon are (1) the excited hollow atom excitation from clusters [34,81] and (2) the relativistic charge-displacement selfchanneling [12-16]. The values of the $(\omega, \mathrm{I}, \mathrm{P})$ triplet required are given approximately by $\hbar \omega \cong 5 \mathrm{eV}, \mathrm{I} \cong 10^{16} \mathrm{~W} / \mathrm{cm}^{2}$, and $\mathrm{P} \cong 1 \mathrm{TW}$.

Three additional physical conditions were also simultaneously satisfied that contributed importantly to the efficacy of the excimer technology in the study of the nonlinear interactions. They are (a) the close match of the optimum wavelength of $~ 200 \mathrm{~nm}$ estimated in Eq.(3) with those characteristic of the $248 \mathrm{~nm}\left(\mathrm{KrF}^{*}\right)$ and $193 \mathrm{~nm}\left(\mathrm{ArF}^{*}\right)$ transitions, (b) the ease of mode-matching the $\sim 2 \mu \mathrm{m}$ focal diameter readily produced with high-brightness $\mathrm{KrF}^{*}(248 \mathrm{~nm})$ technology with the naturally formed plasma channel diameter $(\sim 1.5-2 \mu \mathrm{m})$ that results automatically from the selffocusing action, and (c) the strikingly similar electrostatic force structures [114] associated with the three-dimensional $\mathrm{Xe}(\mathrm{L})$ hollow atom states and the two-dimensional hollow plasma channel. The structural correspondence associated with latter point explains the remarkable compatibility of these two phenomena; specifically, the radiative conditions suitable for the excitation of the channeled propagation are automatically and perforce perfectly fit for the excitation of the $\mathrm{Xe}(\mathrm{L})$ hollow atom states. 
The sextuple confluence formed by the satisfied requirements stemming from the $(\omega, \mathrm{I}, \mathrm{P})$ triplet and the favorable set of the three supplemental physical conditions produced a situation comparable to that of the lucky surfer perfectly positioned on a huge wave; the only possibility is a rapid forward advance propelled by the accumulating momentum of the enormous sea of force behind him. Accordingly, we describe in this section the concepts, design considerations, and architecture of the femtosecond excimer laser technology and conclude with a presentation of predictions concerning its forthcoming scaling to greater peak powers and higher pulse energies.

\subsection{Basic Femtosecond Excimer Laser Properties and Design Logic}

Two distinct options exist for the construction of a terrawatt (TW) femtosecond highbrightness source in the ultraviolet $(\hbar \omega \geq 5 \mathrm{eV})$ spectral region. These alternative approaches are illustrated schematically in Fig.(10). One possible method is the conversion of radiation from a TWclass IR laser directly to the UV with harmonic production, as schematically represented in Fig.(10a). However, it is extremely difficult technically to achieve this frequency conversion, if UV pulse energies up to $\sim 100 \mathrm{~mJ}$ with a femtosecond ( $200 \mathrm{fs}$ ) duration at high brightness are desired. Overall, the barriers to implementation are sufficiently convincing that this method has not been successfully developed.

a)

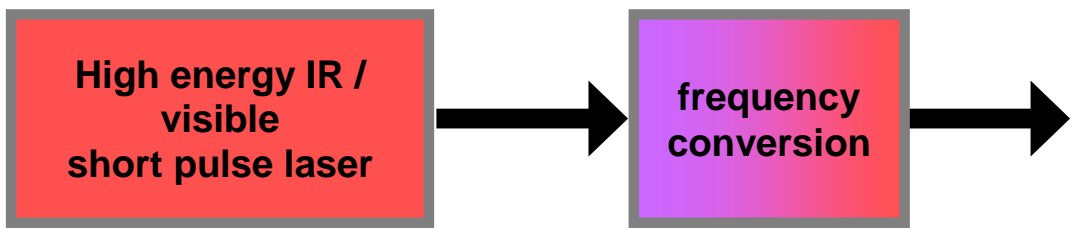

b)

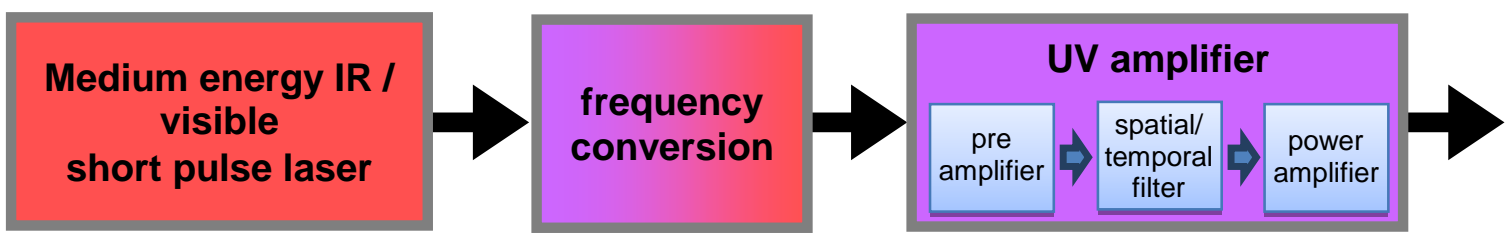

Fig.(10): Two possible approaches for the generation of energetic ultrashort high brightness UV pulses at the TW level. (a) Direct Frequency Conversion of IR to the UV. (b) Hybrid strategy involving the use of a UV seed-beam produced with a femtosecond IR source that subsequently undergoes amplification in excimer amplifier modules.

An alternative hybrid strategy involves the conversion of the radiation of a commercially available IR femtosecond ( $100 \mathrm{fs}$ ) laser system in order to produce a high-brightness UV seed beam that is subsequently amplified by excimer laser modules in the UV, as schematically shown in Fig.(10b). The use of this method enables the wavelength $\lambda$, pulse duration $\tau$, spatial beam divergence $(\Delta \Omega)$, and phase control all to be established with small aperture optics at relatively low energy and power. This situation greatly facilitates the ability to control precisely the characteristics of the system output. Furthermore, the power amplification required to reach the TW regime can be accomplished in the UV with large aperture (10-30 cm diameter) excimer laser amplifiers that are available. However, this approach generally requires the implementation of a system of amplification stages that incorporates suitable processing of the propagating amplified beam to maintain the spatial 
quality of the radiation and obtain high-brightness output pulses. Hence, the architecture of the system must be carefully optimized. This method has enabled the generation of pulse energies of 400-500 mJ at a power of 1-2 TW to be achieved at very high brightness, as further described below.

The media available for amplification in the UV spectral region are few. Hence, the importance of excimer molecules and their properties. Indeed, the only presently known media for the efficient amplification of UV radiation are representatives of the class of rare gas-halide (excimer) gas systems. Excimer lasers derive their emission from molecules that exist only in an electronically excited bound state, as illustrated below in Fig.(32) for the $\mathrm{KrF}^{*}$ excimer. These levels are radiatively unstable and decay rapidly by emission of radiation into a repulsive or loosely bound ground state from which the molecules rapidly dissociate. The transition dipole moment is maximally large, since an electron moves essentially the full interatomic separation of the diatomic molecular state, a property that maximizes the radiation rate. Since the gain of an amplifying transition is directly proportional to the rate of spontaneous emission, this is a very favorable characteristic. In addition, the spectral width of the molecular bound-free transition is large, a property that enables short duration ( 100-200 fs) pulses to be amplified with minimal distortion. The most important molecules employed in commercially available excimer laser systems are $\mathrm{ArF}^{*}, \mathrm{KrF}^{*}, \mathrm{XeCl}^{*}$, and $\mathrm{XeF}^{*}$. Only the $\mathrm{ArF}^{*}(193 \mathrm{~nm})$ and $\mathrm{KrF}^{*}(248 \mathrm{~nm})$ systems were used in the experiments represented in Fig.(1).

The unbound dissociative electronic ground state naturally confers a four-level structure on these systems. Generally, four-level laser media can be operated in a continuous manner. However, this modality of operation is not practically possible with rare-gas halide excimer lasers because of the physical and technical restrictions fundamentally associated with both the ambient high power densities needed for excitation and the likewise mandated large heat transfer rates for cooling.

These technical restrictions relate to basic considerations governing excimer laser operation. Chief among them is the role of the rate of spontaneous emission with respect to the corresponding rate of stimulated emission in the ultraviolet spectral range; specifically, the ratio of the Einstein coefficients $\mathrm{A}$ and $\mathrm{B}$ that is proportional to the third power of the transition frequency $v$ through the relation [115]

$$
\frac{A}{B}=\frac{2 h v^{3}}{c^{2}} .
$$

Obviously, in the ultraviolet range, appreciable amplification through stimulated emission can only compete with losses from spontaneous emission, if the radiation intensity stimulating the transition is sufficiently high. Physically, this requires that the system must be excited at a correspondingly high value of local power density. Consequently, electrical power densities corresponding to greater than 1-2 GW/liter must be deposited in the gas volume. A high specific power density is a fundamental attribute of the excimer media.

In the initial developmental phase of excimer laser technology, the excitation required was furnished by electron beam pumping [116,117]. Presently, however, precisely controlled short-pulse transverse electrical discharges are generally being used, if the aperture of the system has a transverse width less than $\sim 10 \mathrm{~cm}$. An extension of the electrical pulse duration in these discharges beyond approximately $1 \mu \mathrm{s}$ is limited by the development of instabilities that cause arcing, thereby destroying the homogeneity of the excitation and the optical quality of the medium. Furthermore, the repetition 
rate is likewise constrained, since detrimental reaction products and heat must be removed before the next cycle of excitation; accordingly, an exchange of the laser gas is required between pulses.

The electrical energy for the discharge is supplied by a storage capacitor or, more effectively, produced from a low-impedance pulse-forming line. For homogeneous ignition and excitation of the laser volume, a uniform preionization is generally used. Glow discharges, especially in halogencontaining gas mixtures, tend to transform into an arc or unstable sparking discharge due to electron capture by the halogens, so that the laser emission and its spatial uniformity deteriorate rapidly. Hence, considerable effort has been devoted historically to the stabilization of glow discharges $[118,119]$. For example, in order to supply the electrical energy sufficiently fast to avoid the unwanted growth of discharge instabilities, the technique of magnetic pulse compression was introduced, an approach that was implemented mainly with thyratrons serving as the high voltage switches.

From the physical requirements described above, it follows that electrical energy at high specific density, on the order of $64 \mathrm{~J} /$ liter [120], must be delivered homogeneously into the gas medium on a time scale of a few nanoseconds, so that a high-pressure (up to 0.5 MPa) glow discharge can be maintained for a sufficiently long duration. In practice, the discharge terminates after a few tens of nanoseconds. Consequently, the resulting pulse duration of the amplifying state of a standard excimer laser module is of the same order of magnitude, typically 20 - $30 \mathrm{~ns}$.

This limit of the time duration to 20-30 ns has clear implications for the generation of femtosecond amplified pulses. Specifically, it prohibits the customary implementation of the modelocking that would be necessary for the direct generation of the desired ultrashort pulses in the excimer amplifier. Conversely, the $\mathrm{ArF}^{*}$ and $\mathrm{KrF}^{*}$ excimers are especially well suited to the amplification of femtosecond UV seed pulses that are introduced from an independent external source, the configuration illustrated in Fig.10(b). With appropriate excitation, the gain constant of the medium is appreciable, saturation can be achieved yielding favorable energy extraction, and the broad bandwidths of excimer bound-free transitions can support pulse durations down to 100-200 fs, in which the lower limit of the latter parameter arises from gain narrowing. In sum, the natural properties of excimer systems facilitate the generation of (1) high output pulse energies, (2) pulse widths in the femtosecond domain, (3) high peak powers, and (4) very high focal intensities, since the wavelength is short and operation near the diffraction limit is achievable. Laboratory systems can routinely produce a focal intensity of $\sim 10^{20} \mathrm{~W} / \mathrm{cm}^{2}$ at a pulse rate of $\sim 0.1 \mathrm{~Hz}$. A laser system is described below in section 3.4 that exhibits this performance.

\subsection{Short-pulse Amplification Properties of Excimers}

\subsubsection{Gain Dynamics}

Measurements of the gain dynamics have been performed for $\mathrm{XeF}^{*}$ [121], $\mathrm{XeCl}^{*}[122,123]$, and $\mathrm{KrF}^{*}[124,125]$ using the established pump-and-probe technique. These determinations indicated a duration of $\sim 50-90$ ps for recovery of the gain at $78 \%, 65 \%$, and $25 \%$ relative amplitude, respectively, for these three systems. This recovery is attributed to a combined response involving the rotational relaxation within the $\mathrm{B}$-state and the $\mathrm{C} \rightarrow \mathrm{B}$-state relaxation, with a significant additional contribution arising from the relaxation of the ground state for $\mathrm{XeF}^{*}$ and $\mathrm{XeCl}^{*}[121,123]$. It was found that the extractable fraction of the stored energy for pulses shorter than $\sim 5$ ps was $22 \%$ in 
$\mathrm{XeF}^{*}, 38 \%$ in $\mathrm{XeCl}^{*}$, and $75 \%$ in $\mathrm{KrF}^{*}$. These results correspond to the measured values of the saturation energy density corresponding to $\varepsilon_{\mathrm{sat}}=0.2 \mathrm{~mJ} \mathrm{~cm}^{-2}$ [121], $0.85 \mathrm{~mJ} \mathrm{~cm}^{-2}$ [123,126], and 2 $\mathrm{mJ} \mathrm{cm}{ }^{-2}$ [123-125,127], respectively, for these media.

Based on these findings, the $\mathrm{KrF}^{*}(248 \mathrm{~nm})$ system is expected to show similar amplifications for both the short pulse ( 100-200 fs) and the long pulse component comprised of the amplified spontaneous emission (ASE). This fact has great importance for minimizing the ASE background in high-energy amplifier chains. Furthermore, these results solidly confirm that $\mathrm{KrF}^{*}$ is superior to both $\mathrm{XeF}^{*}$ and $\mathrm{XeCl}^{*}$ for short pulse amplification. Fig.(11) illustrates in combined form several important operational characteristics of the $\mathrm{KrF}^{*}$ system.

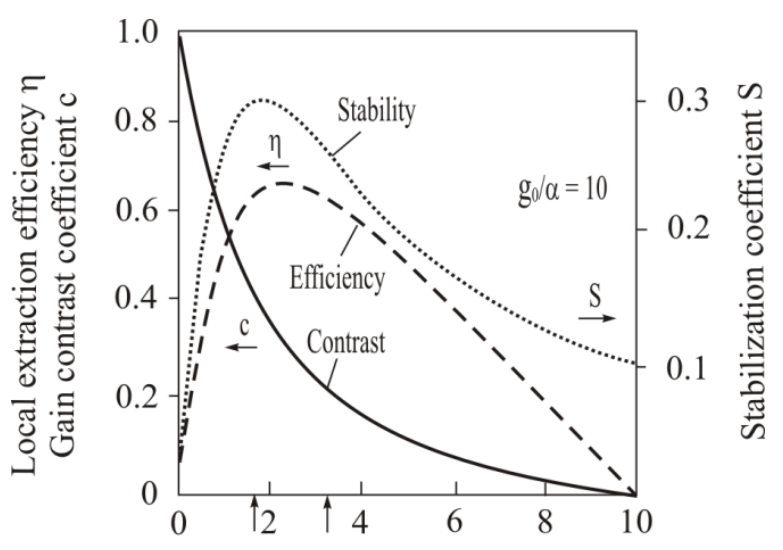

Normalized energy density $\varepsilon\left[\mathrm{mJ} / \mathrm{cm}^{2}\right]$

Fig.(11): Operation characteristics of the $\mathrm{KrF}^{*}(248 \mathrm{~nm})$ amplifying medium. Local extraction efficiency $\eta$ (dashed line), gain contrast coefficient $c$ (solid line), and stabilization coefficient $s$ (dotted line) as a function of the energy density in a $\mathrm{KrF}^{*}$ amplifier. (For the definitions of $\eta, \mathrm{c}$ and $\mathrm{s}$, see [128]). Optimal performance falls in the range of $\varepsilon \cong 2-4 \mathrm{~mJ} / \mathrm{cm}^{2}$.

Since applications generally desire both a maximal pulse energy and a high extraction efficiency, the operation of $\mathrm{KrF}^{*}$ amplifiers must be simultaneously optimized for these two properties. However, it is seen from Fig.(11), which shows the dependence of the extraction efficiency and the gain contrast coefficient on the energy density, that these twin requirements cannot be optimally fulfilled simultaneously. These variables trade-off when operation of the amplifier is close to the saturation energy density. Accordingly, optimized operation can be best attained by an amplifier for which the overall local energy density is limited to a given maximal value, a circumstance corresponding to a limited gain-length product $\mathrm{gL}$, due to the steep slopes of the curves near the optimum range. These physical relationships establish the power limit of an amplifier module.

\subsubsection{Amplification in Media having Non-Saturable Absorption}

Since no effective saturable absorbers are available [129] at the excimer wavelengths, spatial filtering is the easiest way to avoid gain depletion. However, this approach preserves the ASE content 
in the mode of the spatial beam comprising the signal. This ASE content can only be reduced by proper choice of the operational conditions of the amplifier, generally at the expense of efficiency [130-133]. Unfortunately, discharge-pumped $\mathrm{KrF}^{*}$ amplifiers are only efficient when the electrical excitation is fast, necessitating a small discharge loop [134,135]. This constraint limits the crosssection of the discharge, since the system prefers elongated geometries. However, this resulting geometry is not optimal for short-pulse amplifiers possessing significant non-saturable absorption [136-138]. The consideration of these constraints points to a modified architecture for the system.

\subsubsection{Off-Axis and Multiple-Pass Off-Axis Amplification Geometries}

An asymmetrical geometrical configuration can be introduced to optimize the properties for femtosecond pulse amplification. Fig.(12) illustrates the implementation of this arrangement and shows how the effective cross-section of the elongated discharge pumped excimer amplifier is increased while the gain-length product $(\mathrm{gL})$ is simultaneously decreased by tilting the input beam with respect to the geometrical axis of the amplifier [133,139].

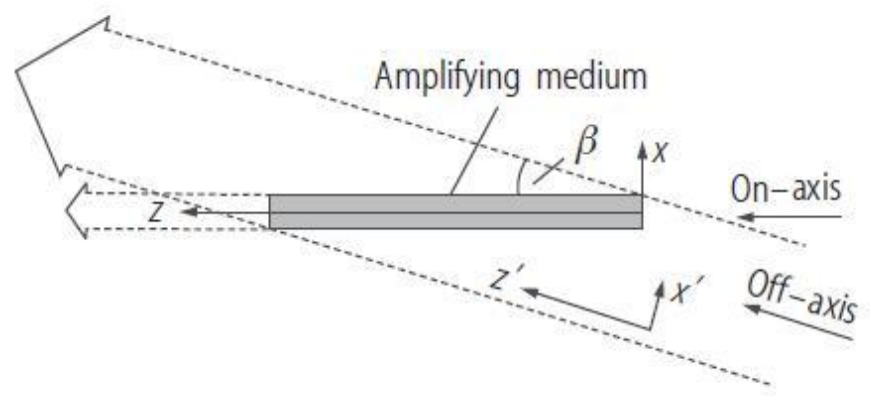

Fig.(12): Illustration of the off-axis amplification geometry that shows the angular tilt $\beta$ between the two axes.

An important property of the off-axis geometry for amplification is that it allows the optimization of the operational conditions, not only for a single amplification pass, but also for multiple pass configurations using the same amplifier module [133,139]. Using a geometry with the appropriate divergence, the beam can be optimally matched to the increased effective cross section of the amplifier by the proper choice of the off-axis angle $\beta$. This configuration enables optimal extraction of the stored energy of the amplifier.

The arrangement shown in Fig.(13) illustrates the practical implementation of a highbrightness UV laser system. In this case, a large-aperture dual-channel $\mathrm{KrF}^{*}$ excimer amplifier is used to boost the final pulse energy to $\sim 60 \mathrm{~mJ}$. Relay imaging of the third-harmonic generation (THG) crystal to the output windows of the subsequent amplifier stages maintains high beam quality. A spatial filter positioned between the discharge tubes provides efficient ASE suppression. The system shown in Fig.(13) can deliver a $(248 \mathrm{~nm})$ pulse energy of $\sim 30 \mathrm{~mJ}$ with a pulse width of $\sim 100$ fs having a final peak brightness exceeding $10^{20} \mathrm{~W} / \mathrm{cm}^{2}$ sr. The use of an $\mathrm{f} / 1.4$ off-axis parabolic mirror to focus the output beam yields the intensity distribution shown in Fig.(14); the peak intensity is $\mathrm{I} \geq 10^{19} \mathrm{~W} \mathrm{~cm}^{-2}$, a value corresponding to an electric field strength of

$\sim 17\left(e / a_{0}^{2}\right) \cong 8.7 \times 10^{10} \mathrm{~V} / \mathrm{cm}$. In the system described below in section 3.4 , this source served as the preamplifier. 


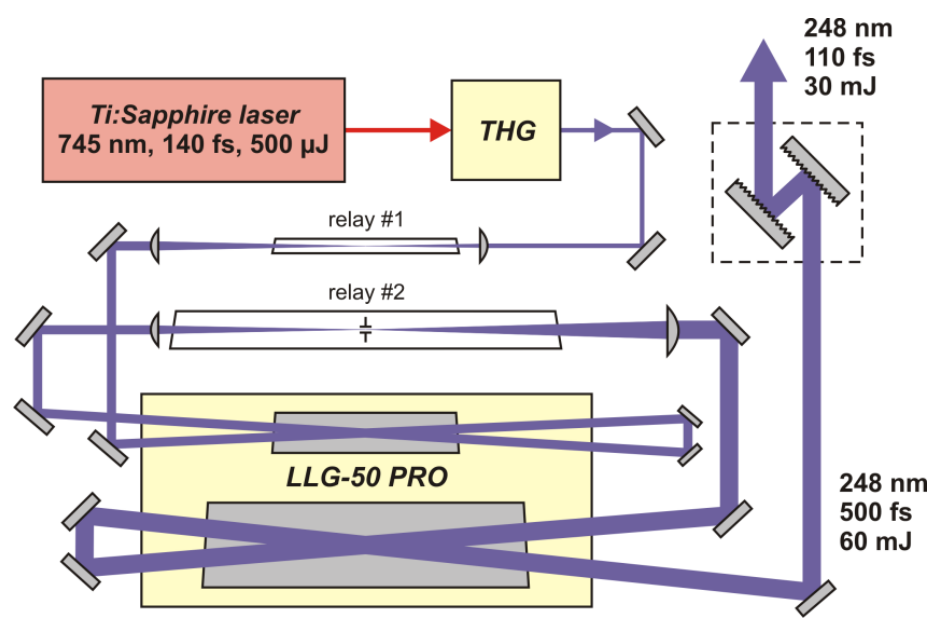

Fig.(13): Schematic of high-brightness $\mathrm{KrF}^{*}(248 \mathrm{~nm})$ laser system that illustrates the major optical components and their spatial arrangements.

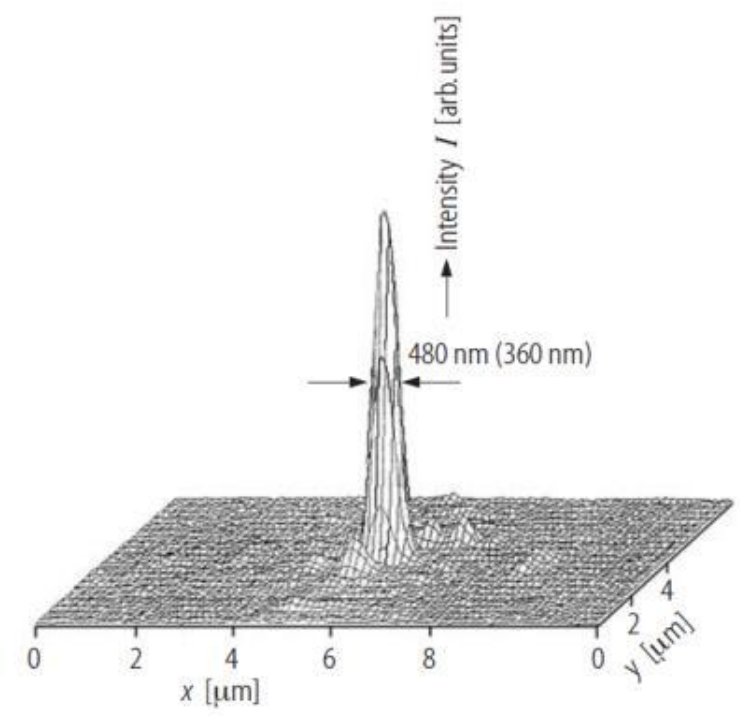

Fig.(14): Intensity distribution of the focal spot of the $\mathrm{KrF}^{*}$ beam from the source shown in Fig.(13) using an $f / 1.4$ off-axis parabolic mirror. The FWHM of the measured distribution is $480 \mathrm{~nm}$ and the diffraction limit is $360 \mathrm{~nm}$. The central spot carries $\geq 60 \%$ of the total energy. 


\subsubsection{Interferometric Multiplexing}

Multiplexing can be employed to optimize the efficiency of energy extraction in amplifier systems. Specifically, due to the short lifetime of the excitation in the excimer medium, successive replenishment of the momentarily stored energy is the only efficient way to extract the entire stored energy [138-141], whose value is given approximately by

$$
\mathrm{E}_{\text {tot }}=\mathrm{E}_{\text {stored }} \mathrm{T} / \tau,
$$

in which $\mathrm{T}$ designates the gain window and $\tau$ represents the recovery time of the gain. Since $\mathrm{T} / \tau \geq 10$ for $\mathrm{KrF}^{*}$, a significant increase of the extractable energy is obtainable by successive depletion of the gain [138]. However, although multiple-pass amplification is technically simpler, in the conventional arrangement for amplification it does not allow the amplifier to operate under the optimum operational conditions for each pass.

The off-axis configuration for amplification [139] significantly ameliorates this problem for discharge-pumped excimer amplifiers $[133,138,139]$ up to a limited number $(\mathrm{n} \approx 3)$ of amplification passes. Since the ideal number of the successive amplification steps in $\mathrm{KrF}^{*}$ amplifiers is $\mathrm{T} / \tau \geq 10$ $[138,142]$, the use of optical multiplexing is clearly necessary to enhance the optical efficiency of the system.

In optical multiplexing, the beam to be amplified is divided into partial beams, which are arranged in the multiplexer to form a pulse train with a separation comparable to the recovery time $\tau$ of the amplifier. The key issue in the multiplexing process is the level of spatial accuracy that can be achieved in the recombination of the partial beams after amplification has been performed. In the conventional multiplexing geometries, this recombination is far from interferometric accuracy, a result that imposes severe limitations on the focusability of the final beam. However, with the use of a properly revised multiplexing method, a rather common optical arrangement can be used both for multiplexing and for demultiplexing [142] that eliminates this problem. Hence, automatic (phaselocked) synchronization of the partial beams can be achieved for any alignment of the multiplexer (demultiplexer), and any form of distortion and/or misalignment is automatically compensated. With this restorative arrangement, optimum focusability can be obtained for the output beam. Indeed, the minimum focal spot size is determined by the diffraction limit of the entire recombined beam $[138,142]$. This significantly increases the source brightness and, accordingly, the maximum focused intensity. Fig.(15) shows a possible realization for the case of two multiplexed beams, using a Sagnac-interferometer with polarization splitting. Furthermore, this arrangement can be adapted to the multiple-pass off-axis amplification geometry [142]. Detailed considerations governing the accuracy of the superposition of the beams and information concerning preliminary experiments for two and four multiplexed beams are presented in Ref.[142]. 


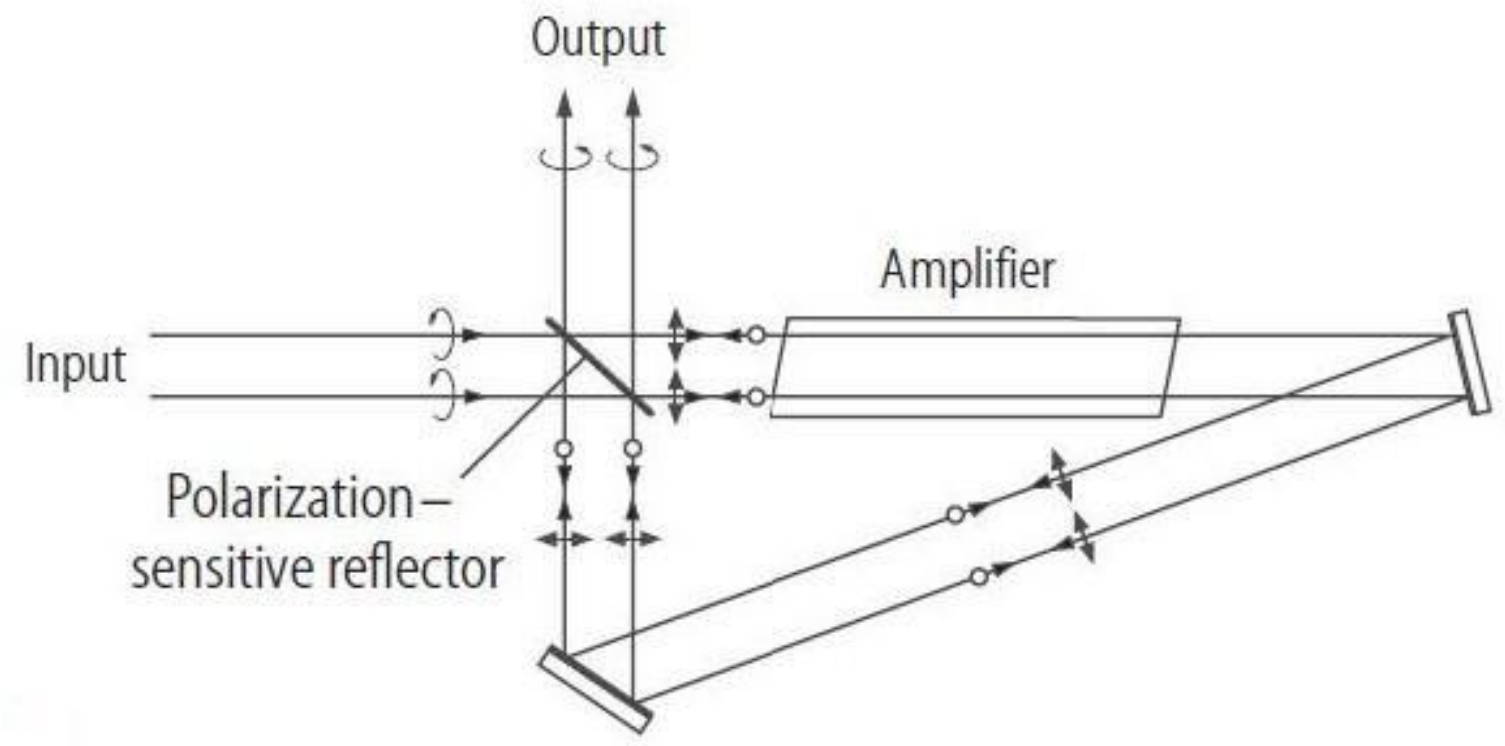

Fig.(15): Illustration of a possible realization of interferometric multiplexing using polarization splitting.

\subsection{Pulse Cleaning Methods}

The configuration shown in Fig.10(b) indicates that short-pulse (femtosecond) excimer laser systems are generally dual-wavelength lasers. Therefore, diffraction-limited beam quality and a welldetermined intensity distribution of the frequency-converted beam are essential. Since phase distortions and intensity modulations are readily magnified in conventional frequency-conversion approaches, the demands on the quality of the frequency converter and the beam spatial profile at the fundamental wavelength are accordingly key. Normally, spatial filtering is a widely used technique to improve the directional properties by allowing the transmission of only the first spatial-component at the position of the Fourier plane. A new special beam filtering method [143] that is based on replacing the pinhole by a nonlinear component, which, due either to the intensity or the energydensity, provides a nonlinear transmission that automatically selects only the intense central spatialcomponent can be employed. This dynamical method automatically generates an output beam with a high-brightness distribution. Moreover, the temporal dependence of the energy flow through the nonlinear element can also be used for spectral modification (spectral filtering) of the pulse [144]. Both spatial and spectral properties of the beam are thereby put under effective and precise control.

Recent development of Ti:sapphire lasers combined with optical parametric amplifiers (OPA) has allowed the technology enabling the generation powerful few-cycle pulses [145] to enter the attosecond regime [146-150]. There are several methods available to reduce the temporal background of these solid-state systems. One effective way is the construction of a laser system with two chirped pulse amplification stages, separated by an intermediate pulse recompression, in which a suitable nonlinear interaction improves the contrast (double CPA, DCPA) [151]. As the effective nonlinear component, a saturable absorber, a nonlinear Sagnac interferometer, a nonlinear ellipse rotator, and, 
the most promising, a cross-polarized wave generation (XPW) process [152-156] have been both suggested and experimentally verified.

However, it is important to note the inherent problems associated with CPA configurations. For example, the relatively high temporal background in the $\sim 100$ ps range of the main pulse is present [155], which has been proven to be critically dependent on the spectral distortions caused by the amplifier chain [157]. The spatial quality is likewise degraded by the limited optical quality of the solid-state material and by the associated dynamic distortions. For these reasons, the temporal profiles of these intense ultrashort laser pulses generally suffer from the presence of prepulses that may originate either from the ASE of the IR or UV amplifier chain, or from the pedestals that arise from imperfect pulse compression. Prepulses are exceptionally detrimental for high-intensity laser-material interactions, since a long prepulse generates a preplasma [158] that significantly alters the interaction and damages the target. In such cases, the main laser pulse interacts not with the intended solid target, but with a preformed plasma. It is shown [159] that even prepulses with an intensity in the range of $10^{7}-10^{8} \mathrm{~W} / \mathrm{cm}^{2}$ can change laser-matter interactions considerably. The net outcome is that the requirements for the temporal contrast scale with the focused intensities. Hence, since the experimentally achieved and the planned intensities are already in the $10^{21}-10^{25} \mathrm{~W} / \mathrm{cm}^{2}$ range, the necessary temporal contrast is set beyond a factor of $10^{13}-10^{18}$.

One of the most efficient and energy-scalable methods used to remove prepulses is based on the self-induced plasma shuttering or plasma mirror technique [160,161], an approach that has also been successfully demonstrated for short-pulse $\mathrm{KrF}^{*}$ laser systems [162]. If the intensity of the laser pulse falling onto a suitable transparent solid material is chosen so that only the leading edge of the main pulse is above the plasma formation threshold, prepulses on the pedestal at lower intensity will be sharply suppressed, thereby improving the contrast up to several orders of magnitude. This method, however, normally allows only a limited contrast improvement and/or limited energy throughput. Moreover, the optical quality of the plasma front influences the phase front of the beam, and a fresh target area is perforce needed for each shot. For these reasons, a novel pulse-cleaning technique is presently introduced below. This approach is generally applicable both for single and dual wavelength lasers, and does not suffer from the shortcomings of the standard plasma mirror method described above.

In the new arrangement, a nonlinear component is situated at the center of a confocal telescope surrounded by a conjugated beam-block filter pair, as shown in Fig.(16).

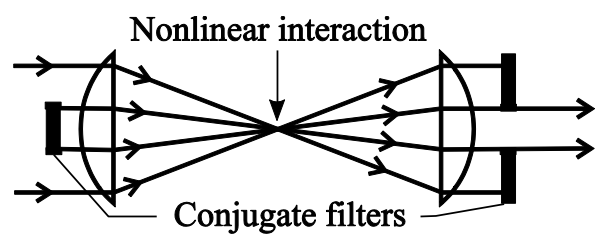

Fig.(16): Schematic of the nonlinear conjugate filter incorporating a nonlinear interaction at the center of the telescope.

The basic concept behind this method is similar to that of active spatial filtering [143]; specifically, an intensity dependent modulation introduced at the Fourier-plane leads to directional modulation. For implementation, an annular input beam, introduced by the input filter with the intensity profile 
shown in Fig.17(a), is ideal, since it has an "Airy-like" intensity distribution in the Fourier-plane (see Fig. 17.b) whose modulation is known to generate a "Gaussian-like" output beam [143]. As long as no modulation occurs in the focal plane, the arrangement corresponding to Fig.(16) has a vanishing transmission, allowing full exclusion of any prepulses. However, for the intense main pulse, which introduces amplitude and/or phase modulation in the focal plane, finite transmission is expected to occur. As described fully below, the overall behavior produced by this arrangement is illustrated by comparison of the spatial profiles illustrated in Figs.(17) and (18).

(a)

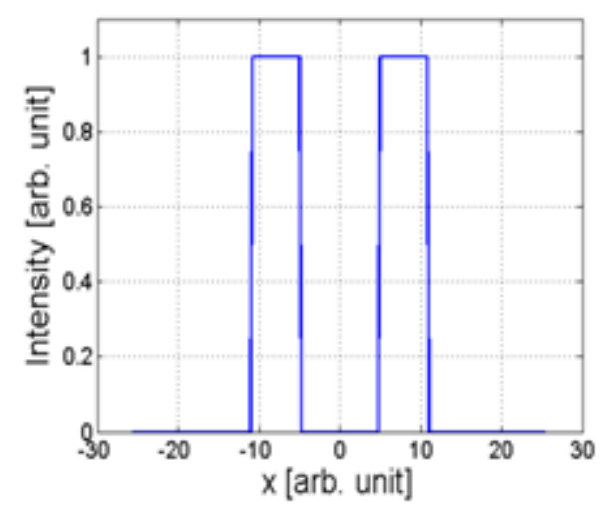

(b)

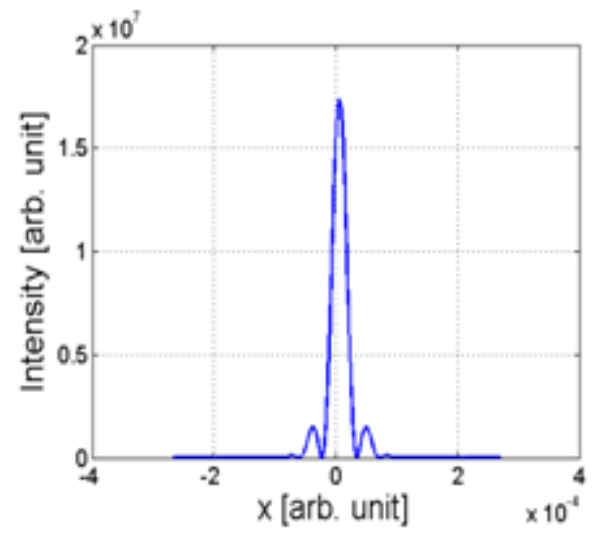

Fig.(17): (a) Cross section of an annular input beam and (b) its corresponding distribution in the focal plane.

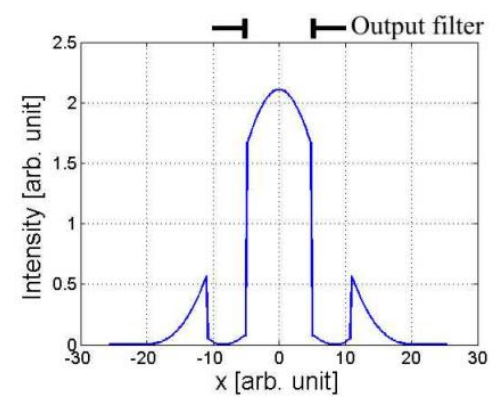

Fig.(18): Output of the nonlinear filter when a phase of the $0^{\text {th }}$ order is shifted by $\lambda / 2$ by the nonlinear interaction. A strong central lobe matching the output filter is shown.

The comparative spatial profiles shown in Figs.(17) and (18) can be readily understood. Indeed, the best results are obtained, together with simultaneous temporal and spatial filtering, when the phase modulation is introduced in the focal plane with no amplitude modulation. Fig.(18) shows the results of the corresponding calculation when the phase of the $0^{\text {th }}$ order is shifted by $\lambda / 2$ by the nonlinear interaction. Practically the "inverse" of the annular input beam shown in Fig.17(a) emerges at the output; as high as 55\% of the energy of the input beam is diffracted to the central hole of the output aperture. Another advantage of this method, beyond the high contrast improvement and high 
overall throughput, is that spatial filtering occurs in the central (transmitted) part of the beam. According to both numerical calculations and corresponding experimental observations, any high order modulation (noise) of the input beam is only present in the low intensity ring-shaped part of the output, which is blocked by the output filter as shown in Fig.(19). The net outcome is that pure phase control produces essentially a perfect beam.

(a)

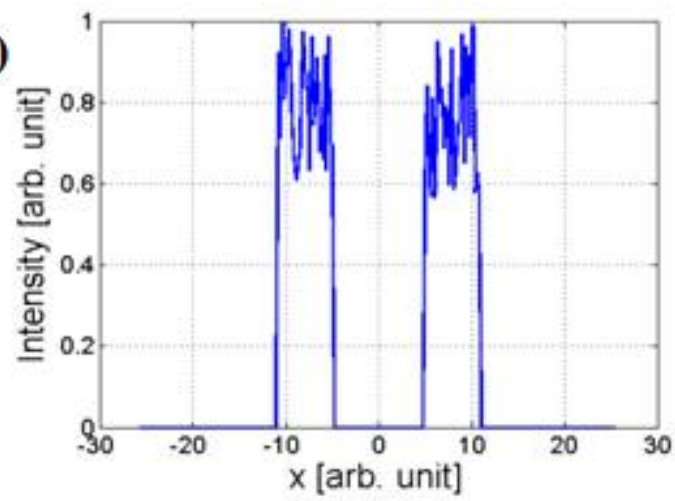

(b)

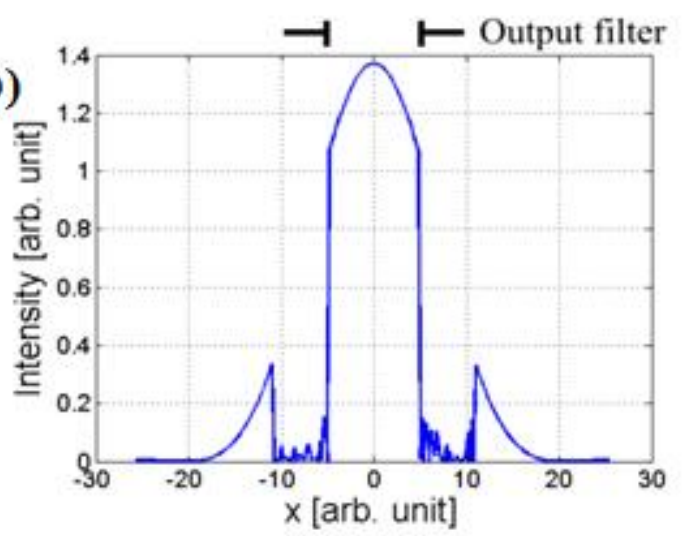

Fig.(19): Input/output characteristics of nonlinear phase controlled filter. (a) Noisy annular input beam spatial profile. (b) Corresponding output beam with a strong central lobe and significantly reduced noise.

Under proper experimental conditions, self-focusing in a self-generated laser plasma produced with a noble gas jet is capable of shifting the phase of the central lobe of the diffraction-pattern by $\lambda / 2$ without significant absorption. Using a pulsed gas jet and a noble gas as the nonlinear phase shifter, constructive interference after passing through the nonlinear medium with a well-defined length allows simultaneously (a) the minimization of the losses and (b) the suppression of the unwanted spatial and temporal components of the laser beam. Importantly, this method is also self-aligning; no matching of the $0^{\text {th }}$ order to a pinhole is needed.

In summary, the main features of the nonlinear plasma filter pictured schematically in Fig.(16) are the following:

- high improvement of the temporal contrast $\left(>10^{3}\right)$, steepening of the leading edge (temporal filtering),

- beam smoothing (spatial filtering),

- self-adjustment (no need for precise alignment),

- very high overall transmission (>40\% obtained experimentally),

- applicable to a broad wavelength range.

It is important to observe that the method based on the phase-shift introduced by the plasma of an ionized noble gas is applicable in a broad wavelength range. However, we note the practical limitation of the application to CPA systems that arises, since the use of the nonlinear temporal filter necessitates the presence of the compressed (minimum) pulse duration, which is normally only 
available at the end of the amplifier chain. On the other hand, with high-intensity excimer systems, due to the direct amplification of femtosecond pulses, the required pulse of short duration is available in all sections of the amplifier chain. Therefore, the use of the nonlinear filter with its high intrinsic throughput, together with saturated operation of the following amplifier(s), leads to a negligible reduction of the output energy of an excimer system.

\subsection{Power and Energy Scalability of High-Brightness Discharge Pumped Excimer Amplifier Systems}

We now turn to issues associated with the power and energy scalability of excimer systems since this issue is directly relevant to the three Grand Challenge Problems introduced in section 4. Since most applications of high-power, high-brightness laser systems require excellent spatial and temporal quality of the pulses, further amplification to boost the output energy from the $100 \mathrm{~mJ}$ level is a sound concept only if the contrast of the pulses can be maintained without significant optical losses or the introduction of excessive optical complexity. With the availability of the new pulse cleaning method described above, we now show that further amplification based on the dischargepumped technology is feasible.

In order to obtain an increase of the short-pulse energy, a corresponding increase of the effective cross-section of the amplifier is necessary. This, however, requires a corresponding increase of the voltage and the transferred energy of the electric pumping circuit. Since, the complexity of the corresponding power system rises sharply above $30-40 \mathrm{kV}$, the discharge gap separation is limited to $\mathrm{d} \approx 4 \mathrm{~cm}$. This bounds the achievable beam cross-section to $\sim 16 \mathrm{~cm}^{2}$ and, therefore, sets a pulse energy limit of $\sim 100 \mathrm{~mJ}$.

We presently describe a solution that extends the energy scaling and thereby enables the short-pulse energy to be lifted to the Joule level. We limit these initial considerations to dischargepumped $\mathrm{KrF}^{*}$ amplifier technology with special emphasis on table-top design, ease of operation, and maximum repetition rate.

We commence this discussion with a specific model represented by a currently functioning system called "PROMETHEUS" that operates in the X-Ray Microimaging and Bioinformatics Laboratory of the University of Illinois at Chicago. The parameters of this system represent the present limit of femtosecond high-brightness discharge pumped $\mathrm{KrF}^{*}$ technology. The power amplifier is a double-discharge gain module with an aperture of $\mathrm{A}=10^{2} \mathrm{~cm}^{2}$ for the discharge crosssection. The performance yields a maximum gain-length product of $\mathrm{g}_{0} \mathrm{~L} \approx 16$ over a duration of $\sim 50$ ns (FWHM). This existing system concept cannot be scaled to a larger effective cross-section. Analysis shows that its stored energy $\mathrm{E}_{\mathrm{m}}=\mathrm{E}_{\mathrm{sat}} \cdot \mathrm{g}_{0} \cdot \mathrm{L} \cdot \mathrm{A}=32 \mathrm{~J}$ is significantly higher than the maximum extractable energy. The maximal amount allowed by the bounded effective beam crosssection $(\mathrm{A})$ is $\mathrm{E}_{\mathrm{out}}=2.2 \mathrm{E}_{\mathrm{sat}} \mathrm{A} \approx 450 \mathrm{~mJ}$ [133]. The explanation of this limitation along with the general consideration of the optimum discharge geometry, with the use of the off-axis amplification modification, is given in Ref.[139].

In Figs.(20) and (21), the beam line overview and corresponding laboratory physical layout are illustrated respectively for the present Prometheus system. The nominal operating parameters are 
power $\mathrm{P} \cong 1-2 \mathrm{TW}$, pulse energy $\mathrm{E}=300-500 \mathrm{~mJ}$, pulse length $200-300 \mathrm{fs}$, and repetition rate $0.1 \mathrm{~Hz}$. With an off-axis $\mathrm{f} / 2$ parabolic mirror on a six-axis stage for precision alignment, as shown in Fig.(22), the system can produce the focal spot of $\sim 2 \mu \mathrm{m}$ in diameter that is presented in Fig.(23). This performance represents $\sim 1.65$ times the diffraction limit and enables focal intensities of $\sim 6-8 \times$ $10^{19} \mathrm{~W} / \mathrm{cm}^{2}$ to be produced, a value that corresponds to an electric field amplitude of $\sim 40\left(\mathrm{e} / \mathrm{a}_{0}^{2}\right)$.

We now illustrate how the current extraction limitation can be reduced and permit the generation of a considerably elevated pulse energy. In order to achieve this improvement, a novel arrangement is introduced that is capable of increasing the optically achievable "effective" crosssection of "rectangular" discharges, essentially improving the aspect ratio associated with the geometry of the discharge. Discharges with an aspect ratio of 1:1 are complicated to create and normally require the use of X-ray preionization. Up to a distance $\mathrm{d} \sim 5 \mathrm{~cm}$ for the gap separation, the standard HV technology based on the use of thyratrons and magnetic pulse compression can be applied. If such a discharge technology is available, the effective cross-section can be multiplied by a factor of 4 by geometrical combination of two discharge sections, as shown in Fig.(24).

\section{Beamline Overview}

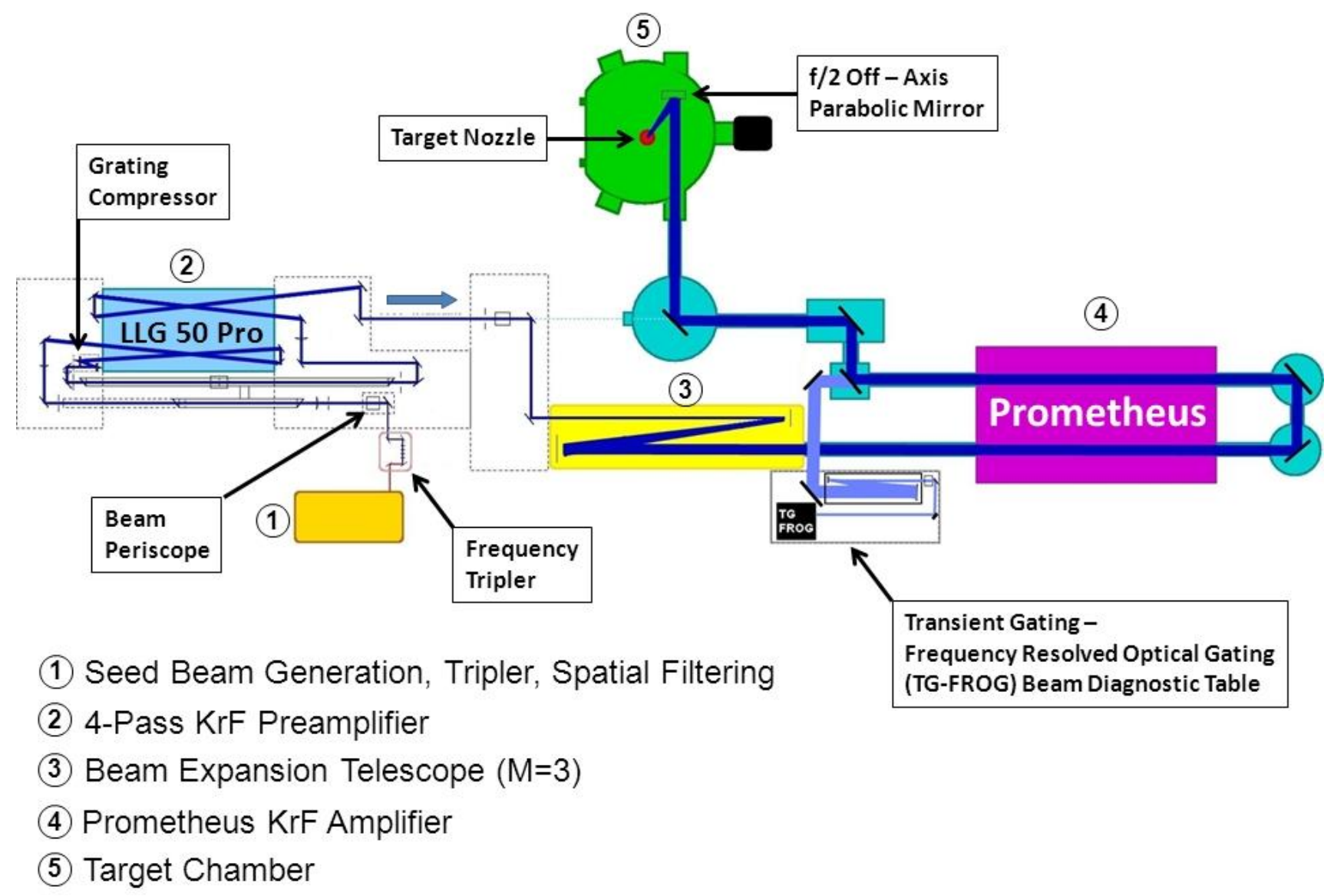

Fig.(20): Schematic presentation of key system components. 


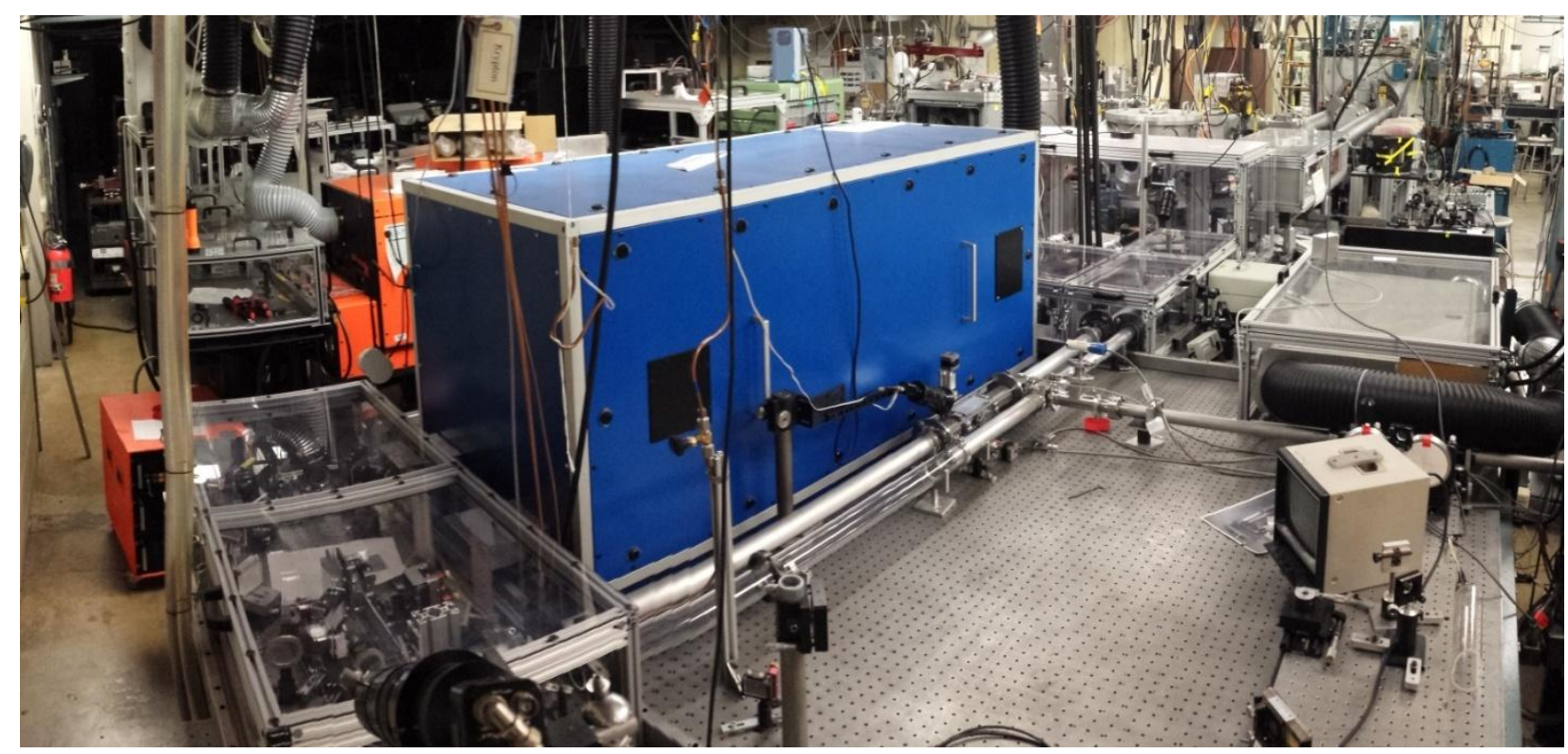

Fig.(21): Presentation of the physical layout of the laser system components. The blue instrument in the foreground is the 4-pass $\mathrm{KrF}$ preamplifier.

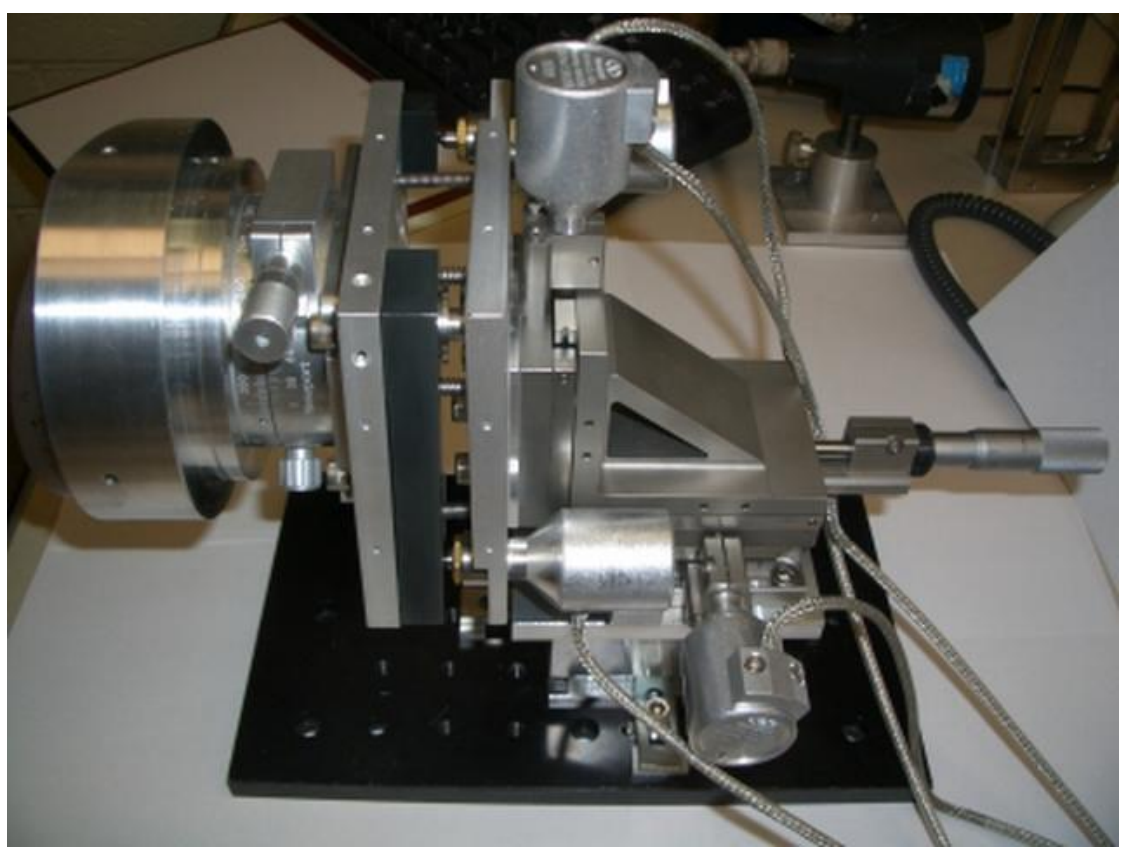

Fig.(22): Controllable 6-axis parabolic mirror mount for f/2 optic with $10 \mathrm{~cm}$ diameter. 


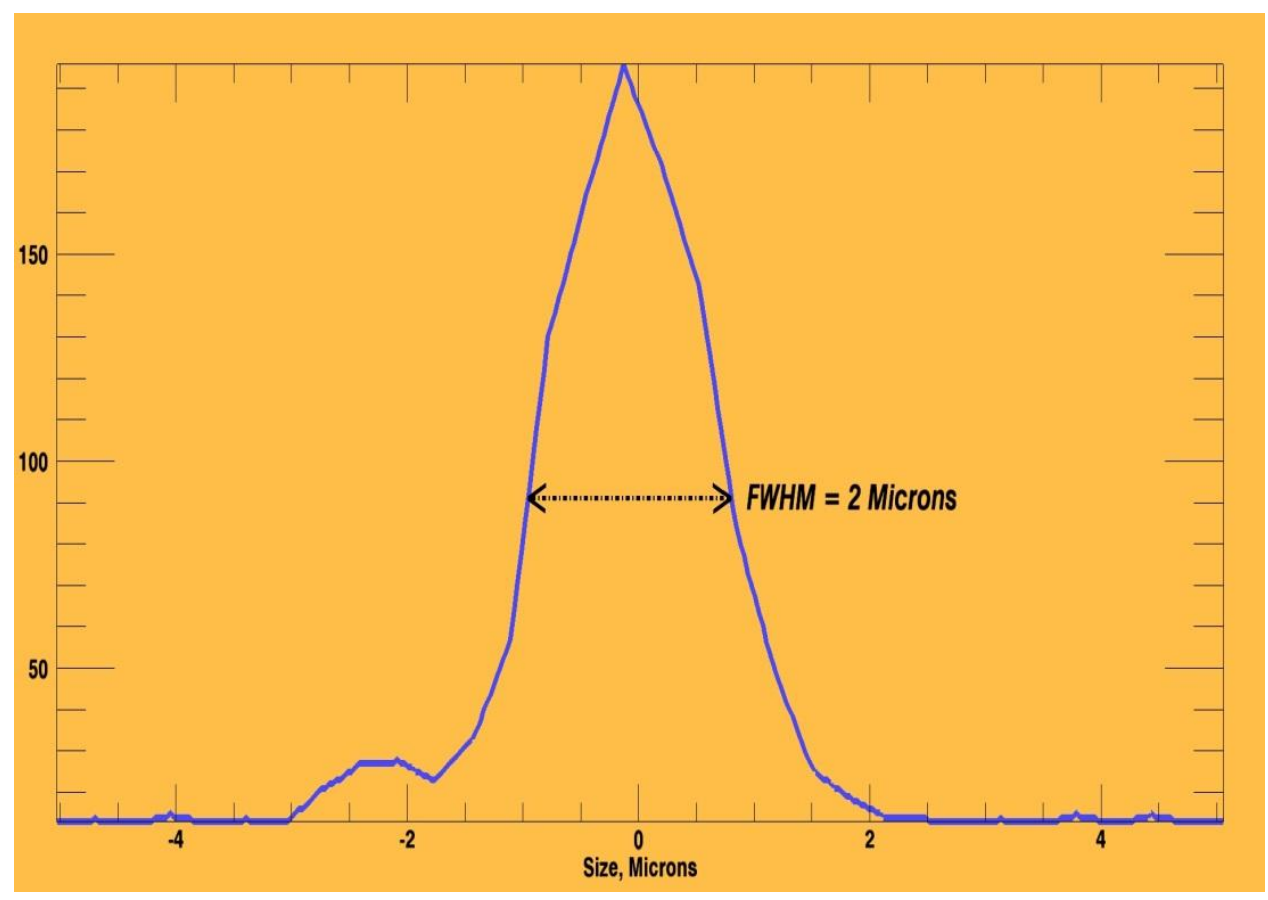

Fig. (23): Measured focal diameter of $\sim 2 \mu \mathrm{m}$ achieved with an $\mathrm{f} / 2$ parabolic optic at a position inside of the experimental chamber. The corresponding theoretical diameter for a Gaussian beam is $\mathrm{D}=1.21$ $\mu \mathrm{m}$. Accordingly, the measurement gives $\sim 1.65$ times the diffraction limit.

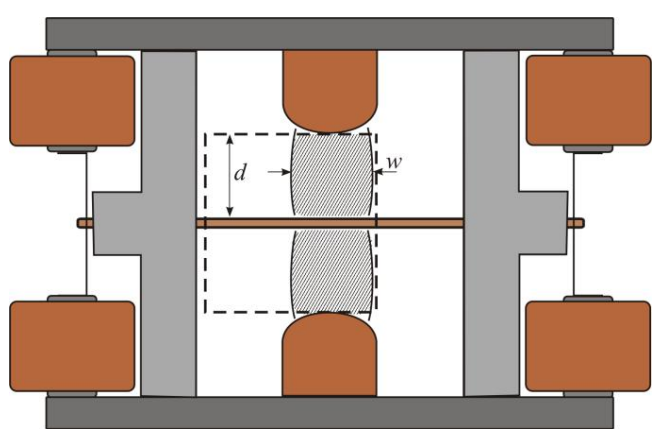

Fig.(24\}: Schematic of the back-to-back design for geometrical combination of two discharges that provides an extended effective distance.

In this configuration, the two discharges are "matched" to each other through their flat electrodes. As a result, in one dimension the combination of the two discharges enlarges the volume, while, in the other dimension, the off-axis geometry increases the effective beam size to be amplified. This corresponds to the dashed line shown in the Fig.(12). The arrangement uses a common window for the two discharges; hence, the effect of the masking from this center" electrode is negligible.

The use of this geometry alters the scaling relationships limitations; specifically, the optically usable cross-section is significantly increased, while the speed of the discharge loop remains unchanged. This modification would raise the achievable energy of a PROMETHEUS-class amplifier to the $\sim 1.5$ Joule range. A further technical advantage is that the two discharge sections can be 
preionized by a common preionizer unit. By the use of the experimentally proven, two-beam interferometric multiplexing of polarization splitting, an additional increase of the final energy, approximately by a factor of 1.7 , is possible [163].

An alternative that would be operable up to an aperture scale of $\sim 30 \mathrm{~cm}$ and enable an additional boost of output pulse energies to the multi-joule level involves the use of the e-beam $\mathrm{KrF}^{*}$ technology that has extensively developed for laser/fusion applications [164]. With the efficiency of kilovolt $\mathrm{x}$-ray production at the demonstrated [26] level of $\sim 30 \%$, $\mathrm{x}$-ray pulse energies of $\sim 1 \mathrm{~J}$ could be anticipated. Pulse energies of this magnitude would be sufficient to apply to the three Grand Challenge Problems introduced in section 4.

\section{Grand Challenge Problems}

The trajectory of advance over the past 50 years serves as a convincing prognostication of future developments. In this section, we briefly introduce three foreseen areas of future study that are perceived as topics of major significance. The landscape associated with these prospective areas is shown in Fig.(25), which is a modestly modified extension of Fig.(1). The essential point shown is illustrated by the ascending blue contour that connects several key findings of the last 50 years and projects predictively upward to the region associated with intensities $>10^{20} \mathrm{~W} / \mathrm{cm}^{2}$, quantum energies $>10^{4} \mathrm{eV}$, and terminates in the zone defined by the Schwinger/Heisenberg Limit. Additional details on the basis of the construction of this curve are given in the supplementary materials of this article with the document identified as "LOB". 


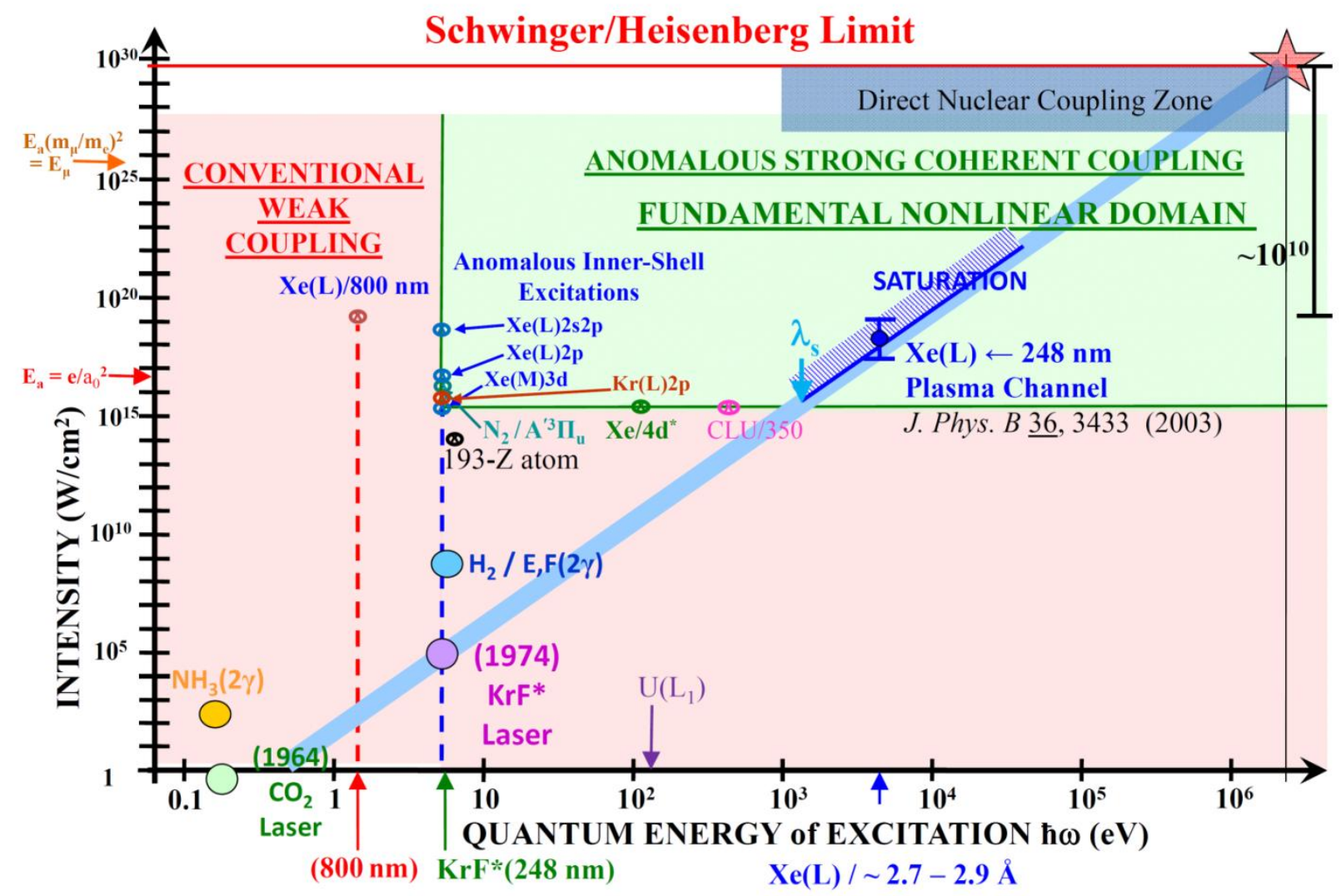

Fig.(25): Fifty year trajectory of advance in nonlinear studies serving as a prognostication of future developments. This graphic, adapted from Fig.(1) and commencing with the $\mathrm{CO}_{2}$ laser $\left(\hbar \omega \cong 10^{-1} \mathrm{eV}\right.$ ), which subsequently extends through the excimer systems (e.g. $\left.\mathrm{KrF}^{*}\right)$ and the $\mathrm{Xe}(\mathrm{L})$ amplifier $(\hbar \omega \cong 4.5 \mathrm{keV})$, presents a physically anchored extrapolation that intersects the zone predicted for direct nuclear coupling and continues to the vicinity of the Schwinger Limit

$\left(\mathrm{I} \cong 4.6 \times 10^{29} \mathrm{~W} / \mathrm{cm}^{2}\right)$ at $\hbar \omega \cong 1 \mathrm{MeV}$, the quantum energy associated with $e^{+} / e^{-}$pair production. The analysis supporting this projection was initially delivered in March 2010 at the "Laser Optics Berlin" conference in March 2010; the power point file identified as "LOB" is contained in the "Supplemental Material" section of this article. The "Time-Line" presented in the "Supplementary Material" provides additional insight into the interpretation of the data.

The Grand Challenge Problems introduced below are (1) a direct experimental probe of the vacuum state with intensities approaching the Schwinger/Heisenberg Limit, (2) the achievement of amplification in the $\gamma$-ray region, and (3) the establishment of a navigable path to the prospective super-heavy Nuclear Island of Stability. The latter two require the creation of conditions suitable for controlled coupling to nuclei while the former demands capability minimally at that level as indicated by the zone of Direct Nuclear Coupling that lies adjacent to the Schwinger/Heisenberg Limit in Fig.(25); the chief outcome is that the physical conditions are comparable for all these problems. Hence, with this consideration in mind, we accordingly provide a preamble that addresses the basis of the potential to achieve nuclear excitation as a introduction to the following discussions on the triplet of specific problems named above. 


\subsection{Nuclear Excitation Preamble/Analogy with Atomic Inner-Shell Excitation}

\subsubsection{Atomic Inner-Shell Excitation}

A striking result of the excitation of Xe clusters with intense $248 \mathrm{~nm}$ femtosecond pulses in selftrapped plasma channels is the copious production of $\mathrm{Xe}(\mathrm{L})$ emission from hollow atom states [34]. The key to the $\mathrm{Xe}(\mathrm{L})$ emission is the selective production of $2 \mathrm{p}$ vacancies in a manner that enables the retention of a subset of electrons in the $3 \mathrm{~d}$ shell, thereby enabling the prompt generation of $\sim 4.4 \mathrm{keV}$ $\mathrm{x}$-rays from $\mathrm{Xe}^{\mathrm{q}+}$ ions on many $(30 \leq \mathrm{q} \leq 37) 3 \mathrm{~d} \rightarrow 2 \mathrm{p}$ transition arrays [38]. Experimental studies have demonstrated several important properties of the mechanism leading to the production of the $\mathrm{Xe}(\mathrm{L})$ hollow atom radiation. Salient among them are (a) the need for cluster formation [33,34], confirmed by the complete lack of x-ray emission from Xe monomer targets under similar conditions of excitation [83], and (b) the inability of comparably intense femtosecond $800 \mathrm{~nm}$ pulses to produce from $\mathrm{Xe}$ clusters the characteristic $\mathrm{Xe}(\mathrm{L})$ hollow atom emissions readily generated with $248 \mathrm{~nm}$ ultraviolet excitation [41,51], as shown in Fig.(1). It is also experimentally established [38] that bright $\mathrm{Xe}(\mathrm{L})$ emission is directly correlated with the production of self-trapped $248 \mathrm{~nm}$ plasma channels [12-16,18,20,23,24,26], since the dynamics of the confined propagation perforce produces a very rapidly ascending leading edge on the pulse whose peak intensity reaches a value of $\sim 10^{20} \mathrm{~W} / \mathrm{cm}^{2}$.

Previous studies have shown how the presence of an ordered phase-sensitive driven electron motion, such as that discussed in section 2.2, can provide an interaction capable of producing the observed behavior [36], a concept initially introduced in an intra-atomic context [28-30]. The present discussion (a) specifically illustrates the attosecond dynamics involved in the $2 p$ vacancy excitation in Xe clusters that yields the $\mathrm{Xe}(\mathrm{L})$ emission and (b), based on this model, introduces the concept of scaling these processes in both frequency and medium density to the neutron-free induction of nuclear fission through the direct coupling to nuclear states with channeled $\mathrm{x}$-rays in high-Z solids. The latter possibility could be considered as the zeptosecond "nuclear echo" of the intracluster attosecond atomic inner-shell vacancy production. Hence, the atomic inner-shell excitation that is experimentally anchored is related to the corresponding nuclear context. This approach thereby furnishes an experimentally based estimate that indicates the feasibility of direct nuclear excitation.

The hollow atom Xe(L) spontaneous emission spectrum, shown in Fig.(26), conspicuously reveals the unusual nature of the excited electronic configurations that characterize these states. Normally, an atomic L-shell spectrum in a highly ionized plasma consists of a few narrow lines; in sharp contrast, the spectrum illustrated in Fig.(26) is a broad doublet that expresses perceptible structure. This atypical emission profile is the canonical presentation of a hollow atom system [108], namely, a high spectral density quasi-continuum of overlapping transitions whose average energy separation is less than the corresponding characteristic radiative width of the lines. For example, the prominently observed $\mathrm{Xe}^{31+}$ array at $\lambda \sim 2.92 \AA$ involves an electronic configuration with a half full $3 \mathrm{~d}$-shell whose corresponding spectrum consists of more than one thousand individual transitions having a radiative breadth of $\sim 1 \mathrm{eV}$ over a full width of the array that is less than $50 \mathrm{eV}$. All of the states producing the spectrum shown in Fig.(26) possess a $2 p$-vacancy and a fraction of the emission in the blue wings of both lobes arises from 2 s2p double vacancy levels $[39,61-63,66]$. 
A previous analysis of the intracluster dynamics [36] attributed the $2 p$-shell ionization to a driven $4 p-2 p$ coherent scattering. We give below dynamical estimates of this interaction as it occurs on the incident edge of a propagating self-trapped $248 \mathrm{~nm}$ pulse in a plasma channel [12-26] in order to illustrate the attosecond nature of this mechanism. Using the development of the first lobe of the $248 \mathrm{~nm}$ wave as a model for the profile of the rapidly rising field strength, the panels shown in Fig.(27) illustrate the sequence of interactions involved in this intracluster process.

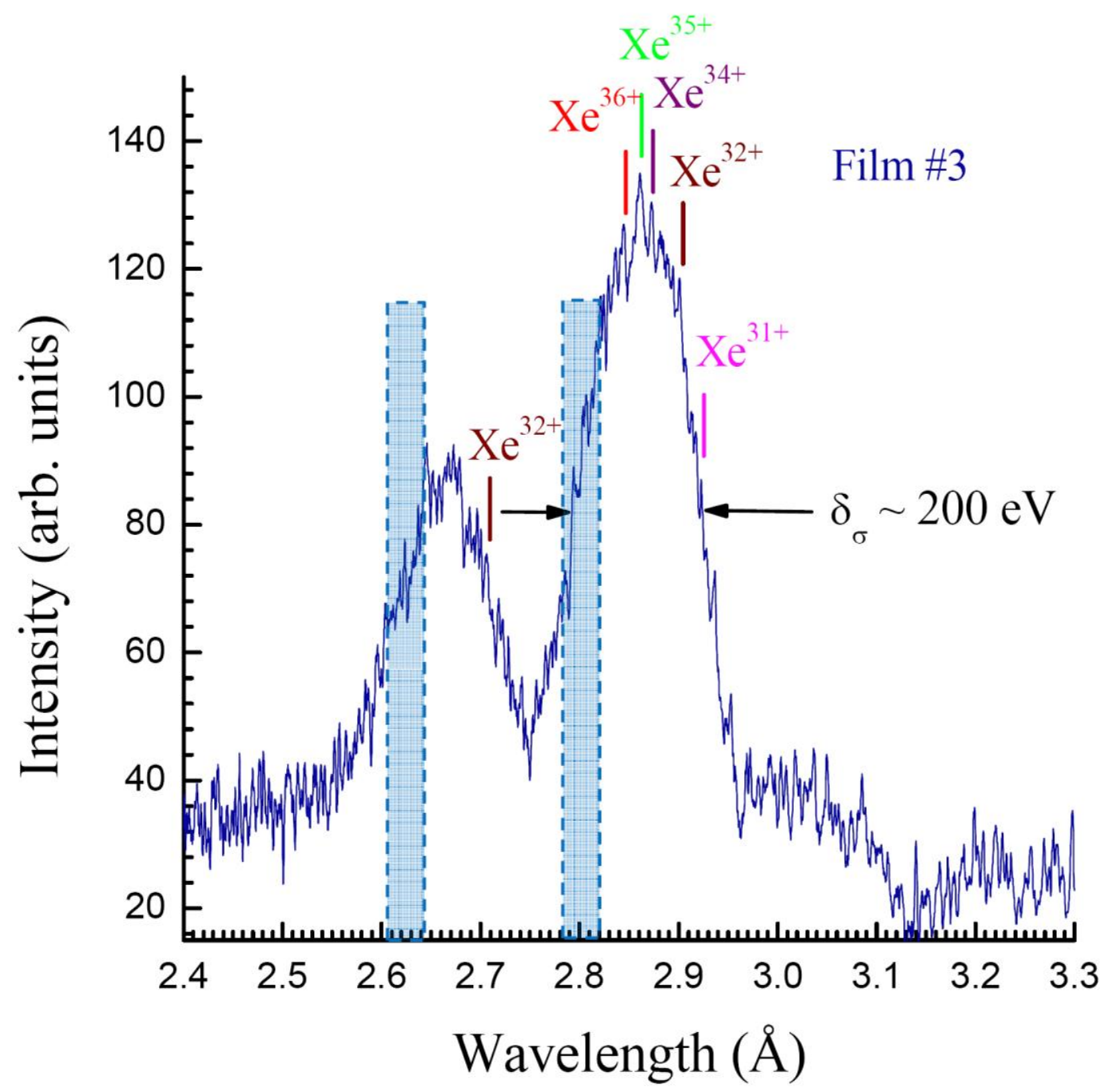

Fig.(26): Characteristic $\mathrm{Xe}(\mathrm{L})$ hollow atom spontaneous emission spectrum excited in the absence of plasma channel formation by intense $\left(\geq 10^{17} \mathrm{~W} / \mathrm{cm}^{2}\right)$ femtosecond $248 \mathrm{~nm}$ pulses that is composed of several $\mathrm{Xe}^{\mathrm{q}+}$ charge state arrays that emit on $3 \mathrm{~d} \rightarrow 2 \mathrm{p}$ transitions in the wavelength region $\lambda \sim 2.8 \AA$. The positions of selected $\mathrm{Xe}^{\mathrm{q}+}$ charge state arrays are shown; the blue shaded zones on the blue wings of the doublet features designate the spectral regions associated with transitions from $2 \mathrm{~s} 2 \mathrm{p}$ double vacancy states [61]. For further spectral details, see Refs. [38,39,61-63,66]. 

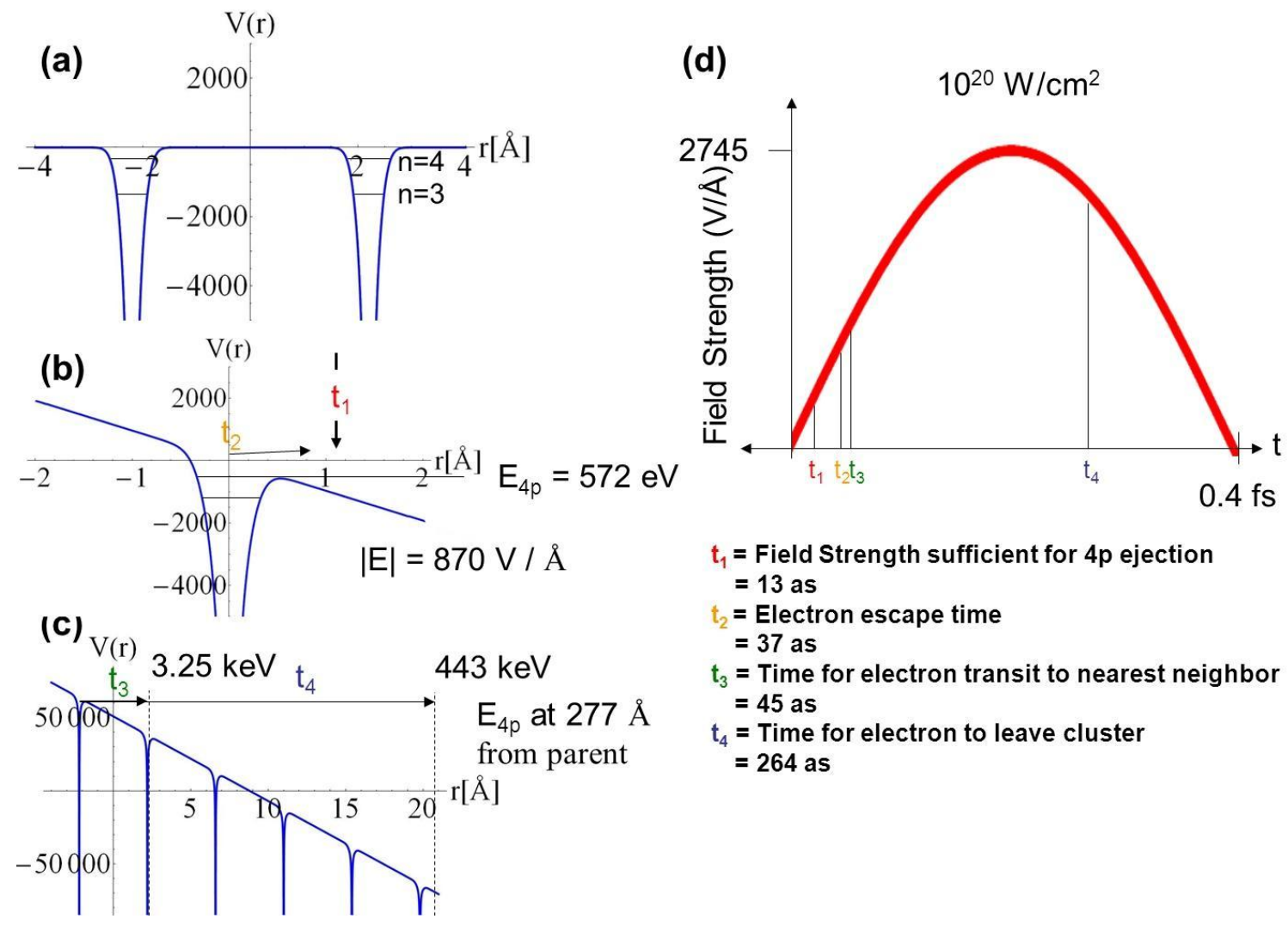

Fig.(27) : The sequence of dynamical processes in the mechanism of $2 p$ inner-shell ionization by a driven $4 \mathrm{p}-2 \mathrm{p}$ collisional interaction with a pulse of $248 \mathrm{~nm}$ radiation whose rapidly rising incident edge is dynamically generated by self-trapped propagation in a plasma channel is portrayed. The peak intensity achieved is estimated to be $\sim 10^{20} \mathrm{~W} / \mathrm{cm}^{2}$. (a) Electronic potentials $\mathrm{V}(\mathrm{r})$ as a function of radial distance in $\AA$ units associated with two adjacent atoms in a Xe cluster are represented. The potentials shown correspond to atomic structural information derived from the analyses of Herman and Skillman [165]. Atomic subshell ionization data are given in Ref.[166]. (b) With the electric field amplitude shown in panel (d), that depicts the fast rise of the corresponding $248 \mathrm{~nm}$ intensity that develops on the leading incident edge of the propagating pulse, the estimated time for the Starkinduced continuum lowering to release a $4 p$ electron with a binding energy of $\sim 572 \mathrm{eV}$ is estimated to be $t_{1} \cong 13$ as. The corresponding time estimated for the escape of the unbound $4 p$ state from the central region of the atom is $t_{2} \cong 37$ as at a field strength of $870 \mathrm{~V} / \AA$. (c) The transit of the $4 \mathrm{p}$ electron accelerated by the optical field through the Xe cluster is represented. The nearest-neighbor electronic interatomic transit time $t_{3} \cong 45$ as and a cluster transit time $t_{4} \cong 264$ as are estimated. The sums $\tau=t_{1}+$ $t_{2}+t_{3}=95$ as and $T=\tau+t_{4}=359$ as represent the nearest-neighbor interatomic and cluster time scales, respectively, the latter for a cluster nominally containing $\sim 100$ atoms. (d) The contour of the electric field amplitude of the pulse on the leading edge of the $248 \mathrm{~nm}$ wave propagating in a selftrapped plasma channel is depicted. The maximum intensity is $\sim 10^{20} \mathrm{~W} / \mathrm{cm}^{2}$, the pulse peak power is $\sim 1 \mathrm{TW}$, and the plasma density of the channeling medium is $\sim 10^{21} \mathrm{~cm}^{-3}$, a level that is $\sim 5 \%$ of the critical plasma density at $248 \mathrm{~nm}$. 
Four time scales are identified in Fig.(27) that characterize the field ionization, interatomic transport, and exit of the electron from the cluster. The key time associated with the $2 p$ vacancy production from the nearest neighbor electronic coupling is

$$
\tau=\mathrm{t}_{1}+\mathrm{t}_{2}+\mathrm{t}_{3} \cong 95 \text { as, }
$$

the interval that governs the collision interaction time of the free $4 p$ electron liberated from one atom with the bound $2 p$ electron in the adjacent system. Linear polarization of the $248 \mathrm{~nm}$ radiation is assumed so that the incident wave communicates no angular momentum to the electron in this interaction. We note that the time found for $t_{2}$ of 37 as is fully consistent with the moderately longer intervals that are naturally expected to be characteristic of the tunneling regime [167]. We note that the work of Pfeiffer et al. [168], that involves a more complicated experimentally tested model of electron escape, yields comparable dynamical times. With the canonical atomic time $\tau_{\mathrm{a}} \cong 24$ as, we have

$$
\tau / \tau_{\mathrm{a}} \cong 4
$$

hence, $\tau$ corresponds equivalently to an energy of $\sim 6.8 \mathrm{eV}$, a value that is only slightly above the quantum energy $(\hbar \omega=5 \mathrm{eV})$ associated with the incident wavelength of $248 \mathrm{~nm}$.

An earlier analysis [42] showed that the presence of an ordered phase-sensitive interaction could be correlated with the $\mathrm{L}_{1}$-subshell Auger width of atoms [92-94]. Specifically, if the inverse of this Auger width is used as a measure of the electron-electron interaction dephasing time, the experimentally established minimum in the $\mathrm{L}_{1}$ level widths in the atomic number range $50 \leq \mathrm{Z} \leq 70$ favors $\mathrm{Xe}(\mathrm{Z}=54)$ atoms for a coherent interaction of the envisioned type. The corresponding $\mathrm{L}_{1}-$ width for $\mathrm{Xe}$ is [42]

$$
\Gamma=3-4 \mathrm{eV},
$$

a range corresponding to a characteristic time $\tau_{\mathrm{A}} \sim 159-216$ as that yields the result

$$
\tau<\tau_{\mathrm{A}}
$$

It follows from Eq.(17) that the estimated time scales of the interactions illustrated in Fig.(27) are consistent with the requirements for an ordered phase-sensitive interaction that can selectively generate $2 p$ vacancies in Xe. We also note that, given the characteristic attosecond times represented in Fig.(27), sufficiently sensitive measurements of high harmonic generation in clusters [169] could potentially provide complementary information on these dynamics.

\subsubsection{Extension to Direct Nuclear Excitation}

The stable physical mechanism that compresses radiative energy into narrow channels [12$16,18,20,23,24,26]$ with the propagation of $248 \mathrm{~nm}$ pulses in self-guided under-dense channels at a plasma electron density of $\rho_{\mathrm{e}} \sim 10^{21} \mathrm{~cm}^{-3}$, and thereby dynamically generates an extremely sharply rising incident wave profile, admits the possibility of scaling the interaction to greatly reduced time scales at shorter wavelengths, higher plasma densities, enormously augmented power densities, and 
nuclear interactions [102]. This scaling of the self-channeling phenomenon from the ultraviolet region $[12-16,18,20,23,24,26]$ to the $x$-ray range is called "Photon Staging" [100-102]. This step represents a transition in parameter space that raises the quantum energy $\hbar \omega$ into the multikilovolt regime and the plasma density to $\rho_{\mathrm{e}} \sim 4.4 \times 10^{24} \mathrm{~cm}^{-3}$ in the case of solid uranium. The resulting correspondence is that both $\omega$ and $\rho_{\mathrm{e}}$ are increased approximately by a factor of $\sim 10^{3}$ and leads to the production of channels with characteristic diameters of $\sim 100 \AA$, a dimension less than the size of a typical virus. In general terms, the creation of such channels with multikilovolt $\mathrm{x}$-rays in high-Z solids yields projected power densities $[100,102]$ on the order of $\sim 10^{28}-10^{30} \mathrm{~W} / \mathrm{cm}^{3}$. In order to illustrate the characteristic intensities and time scales of this scaling, we will use the example of nuclear fission $[103-105,170]$ generated in an $\mathrm{x}$-ray channel that is produced in solid uranium.

Previous calculations [102] of self-trapped channels of $\mathrm{Xe}(\mathrm{L})$ radiation at $\hbar \omega \cong 4.4 \mathrm{keV}$ in solid uranium provide the information required to estimate the zeptosecond time scale to reach an intensity of $\sim 10^{27} \mathrm{~W} / \mathrm{cm}^{2}$, the level associated with the projected onset of direct coupling [102] of the incident radiation to nuclear matter shown in Fig.(28). The peak intensity generated in these channels [102] is $\sim 10^{29} \mathrm{~W} / \mathrm{cm}^{2}$. Using the same procedure that produced the intensity profile illustrated in Fig.27(d), the resulting envelope of the leading edge of the channeled x-ray pulse, as shown in Fig.(28), gives a rise time of $\tau_{\mathrm{x}} \cong 15 \mathrm{zs}$ to the intensity $\mathrm{I} \cong 10^{27} \mathrm{~W} / \mathrm{cm}^{2}$. This interval is compared in Fig.(29) to the time scales associated with the process of nuclear fission. We observe that the scission dynamics can occur nominally in the $\tau_{\mathrm{f}}=10-30 \mathrm{zs}$ range $[171,172]$, thereby giving the approximate equivalence

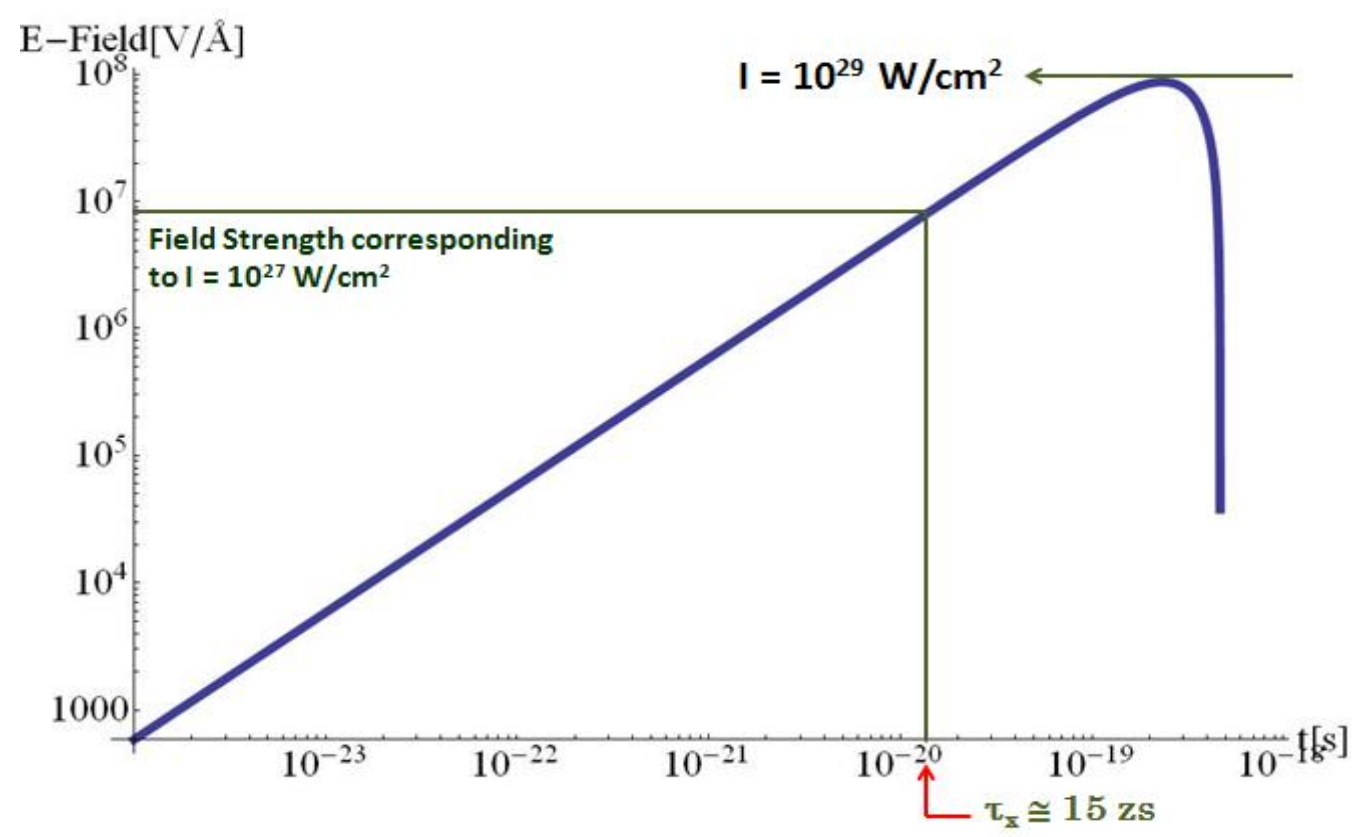

Fig.(28): Electric field profile of the incident leading edge of a self-trapped $2.8 \AA \mathrm{x}$-ray pulse in solid uranium [102]. As illustrated, the peak intensity produced by the pulse is $\sim 10^{29} \mathrm{~W} / \mathrm{cm}^{2}$ and the intensity of $\sim 10^{27} \mathrm{~W} / \mathrm{cm}^{2}$, the value estimated for direct radiative coupling to nuclear states, is reached in a time of $\tau_{\mathrm{x}} \cong 15 \mathrm{zs}$. 


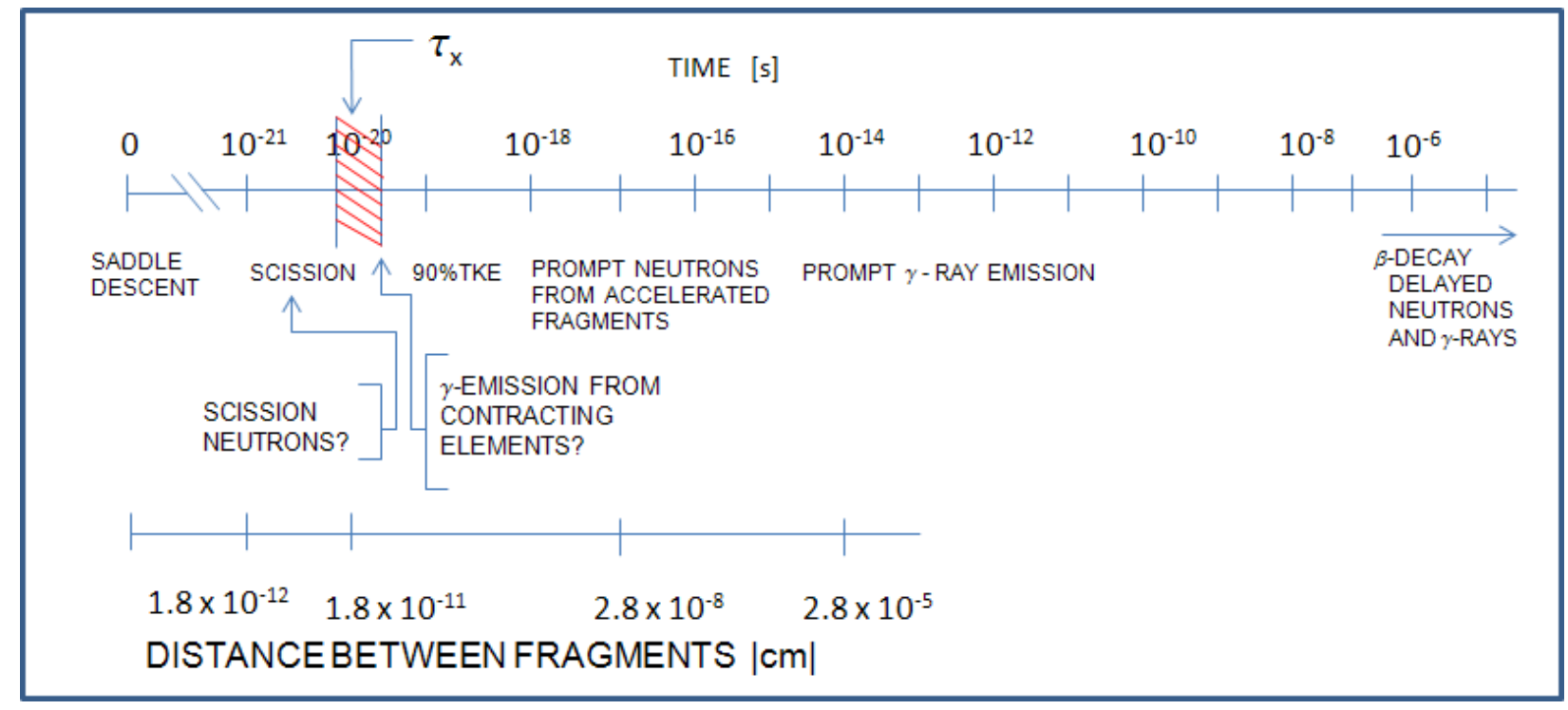

Fig.(29): Estimated time scales associated with the evolution of the nuclear fission process. The key scission stage [172] occurs over an interval of $\sim 10-30 \mathrm{zs}$. The value $\tau_{\mathrm{x}} \cong 15 \mathrm{zs}$ from Fig.(28) is indicated along with the shaded range of $10-30 \mathrm{zs}$ for the parameter $\tau_{\mathrm{f}}$. Figure adapted from HansHermann Knitten, Ulrich Brosa, and Carl Budtz - Jørgensen, "Neutron and Gamma Emission in Fission," in The Nuclear Fission Process, edited by Cyriel Wagemans (CRC Press, Inc., Boca Raton, FL, 1991) p. 497. Figure used with permission.

$$
\tau_{\mathrm{x} \lesssim} \tau_{\mathrm{f}}
$$

a relationship that mirrors Eq.(17), given our present level of knowledge of the details of nuclear dynamics [173]. We also note that the corresponding nuclear dimensional size $\delta$ during this critical stage of nuclear evolution on the reaction coordinate is $\delta \sim 7-25 \times 10^{-12} \mathrm{~cm}$. Certain heavy strongly deformed triaxial nuclei that possess multiple and complex fission pathways [174] may be favorable candidate systems for the observation of this direct nonlinear nuclear process, since the gross nuclear spatial reorganization required to reach the fission exit configuration is expected to lengthen the dynamical time scale beyond that shown for $\tau_{\mathrm{f}}$ in Fig.(29), thereby strengthening the inequality given by Eq.(18).

The fast pulse rise time that is perforce dynamically developed by the self-trapped propagation of $248 \mathrm{~nm}$ pulses in under-dense plasma channels induces an attosecond scale interatomic electronic motion in Xe clusters that produces an ordered phase-dependent interaction capable of selectively generating hollow atom states with 2 p-vacancies. Estimates indicate that this dynamical behavior may potentially be extended into the nuclear zeptosecond region with the concept of "Photon Staging", a process that is the analogous production of self-trapped channels in high-Z solids with $\mathrm{x}$-rays. 


\subsection{Discussion of Grand Challenge Problems}

\subsubsection{Direct Probe of the Vacuum State}

The properties of the vacuum state have been propelled to the zenith of physical importance by the experimental discovery $[175,176]$ of a nonvanishing Cosmological Constant $\left(\Omega_{\Lambda} \cong 0.73\right)$ that embodies a universally pervasive "Dark Energy". A dynamic probe of this entity would doubtlessly yield many informative findings giving insight on the nature of the vacuum. If appropriately developed laser technology could reach an intensity in the vicinity of the Heisenberg/Schwinger Limit $[98,99,177]$ of $\sim 4.6 \times 10^{29} \mathrm{~W} / \mathrm{cm}^{2}$, explosive $e^{+} / e^{-}$pair production is expected. However, studies [178-183] indicate that the achievement of intensities as low as $\sim 10^{25} \mathrm{~W} / \mathrm{cm}^{2}$ would yield detectable signals stemming from the influence of vacuum polarization. Hence, experimental intensities in the $10^{25}-10^{29} \mathrm{~W} / \mathrm{cm}^{2}$ range, a span that fully overlaps the zone designated as "Direct Nuclear Coupling" in Fig.(25), are indicated as sufficient to serve as a probe of the character of the vacuum state.

Two different technological proposals are presently under evaluation. One involves large infrared $(\lambda \sim 1 \mu \mathrm{m})$ lasers, but faces a serious limit from electron cascades [97]. A second approach [102] utilizes X-rays, involves a concept known as "Photon Staging", and is immune to the electron cascade phenomenon. Studies have shown that the use of $\mathrm{x}$-rays $[102,184,185]$ has the advantage that the requisite intensities may be reached with a pulse energy as low as $\sim 100 \mathrm{~mJ}$. Accordingly, this approach has the advantage of small scale, relatively low cost, and a correspondingly more rapid rate of implementation. In connection with the approach involving x-rays, we outline below the concept of "Photon Staging". The detailed description of these proposed efforts concerning the study of the vacuum state are contained in Refs. [102,184,185].

The use of coherent $\mathrm{x}$-rays for the attainment of intensities approaching the Schwinger Limit [99] of $\sim 4.6 \times 10^{29} \mathrm{~W} / \mathrm{cm}^{2}$, a condition initially discussed by Sauter [177] and Heisenberg and Euler [98], is supported by very powerful scaling relationships. The key concept for the production of ultrahigh intensities with x-rays is "Photon Staging". Simply stated, this is the channeling process outlined in Fig.(30) elevated in both frequency $\omega$ and electron density $\mathrm{n}_{\mathrm{e}}$. The governing scalings are illustrated in Fig.(30); basically, it is the channeling of x-rays in solids, a phenomenon that raises the critical power $\mathrm{P}_{\text {cr }}$ to values into the range of $\sim 0.1-1 \mathrm{PW}$. Since the maximum plasma density is $\mathrm{n}_{\mathrm{e}} \sim 4$ $-5 \times 10^{24} \mathrm{~cm}^{-3}$ in high-Z solids, the corresponding channel diameters are compressed to $\sim 100 \AA$ in materials like $\mathrm{Fe}, \mathrm{Au}$, and $\mathrm{U}$. This nonlinear focusing mechanism (1) obviates the need for x-ray optics, (2) has demonstrated [26] an efficiency in the ultraviolet of $>90 \%$, and (3) produces a focal diameter that is governed mainly by the plasma density. The key requirement [12-16] for the production of a channel in the underdense regime is the generation of a peak power $\mathrm{P}_{\mathrm{o}}$ exceeding the critical power $\mathrm{P}_{\mathrm{cr}}$ necessary for the development of the confined propagation with the relativistic/charge-displacement mechanism. The overall scaling of this mechanism is such that, for $\mathrm{x}$ rays with $\hbar \omega>1 \mathrm{keV}$ in any solid target, the onset of channeled propagation perforce generates an intensity of $\sim 10^{26} \mathrm{~W} / \mathrm{cm}^{2}$, or greater. Hence, these dynamics strongly favor the production of intensities in the desired range. 


$$
\begin{aligned}
& \text { - } \hbar \omega=5 \mathrm{eV}(248 \mathrm{~nm}) \longrightarrow \hbar \omega_{\mathrm{x}} \cong 4500 \mathrm{eV}(2.8 \AA) \\
& \text { - } \mathrm{n}_{\mathrm{e}} \cong 10^{21} \mathrm{~cm}^{-3} \longrightarrow \mathrm{n}_{\mathrm{e}} \sim 10^{24}-10^{25} \mathrm{~cm}^{-3} \text { (Solid) }
\end{aligned}
$$

\section{CRITICAL POWER SCALING}

$$
\begin{aligned}
\mathrm{P}_{\mathrm{cr}} & =\left(\mathrm{m}_{\mathrm{e}, 0}^{2} \mathrm{c}^{\left.5 / \mathrm{e}^{2}\right)} \int_{0}^{\infty} \mathrm{g}_{0}^{2}(\rho) \rho \mathrm{d} \rho\left(\omega / \omega_{\mathrm{p}, 0}\right)^{2}\right. \\
& \cong 1.6198 \times 10^{10}\left(\omega / \omega_{\mathrm{p}, 0}\right)^{2} \mathrm{~W}
\end{aligned}
$$

OUTCOME

$$
\begin{aligned}
& \omega \longrightarrow 10^{3} \omega \\
& \mathrm{n}_{\mathrm{e}} \longrightarrow\left(10^{3}-10^{4}\right) \mathrm{n}_{\mathrm{e}}
\end{aligned}
$$

- $\mathrm{P}_{\mathrm{cr}} \cong 1 \mathrm{PW}(2.8 \AA) \rightarrow 10$ millijoules in $\sim 10$ as

Fig.(30): "Photon Staging", channel formation in solids with x-rays. Plasma channel formation becomes possible with pulse energies of $\sim 10-100 \mathrm{~mJ}$ for corresponding X-ray pulse lengths of $\sim 10$ - 100 as. Enormously enhanced power compression is the leading outcome. The exact expression for the critical power for relativistic and ponderomotive self-channeling $\mathrm{P}_{\mathrm{cr}}$ has been derived in Ref.[13].

The stability of the propagation is assured by the existence of a robust eigenmode $[13,15,16,23,25,26]$. For the case of $\lambda_{\mathrm{x}} \cong 2.9 \AA$ and fully ionized uranium, $\mathrm{P}_{\mathrm{cr}} \cong 49 \mathrm{TW}$. With a pulse length $\tau_{\mathrm{x}} \sim 50$ as, a value that is well within the projected performance $[65,66]$ of the $\mathrm{Xe}(\mathrm{L})$ system, the critical power corresponding to uranium can be achieved with a pulse energy as small as $\mathrm{E}_{\mathrm{x}} \sim 3.0$ $\mathrm{mJ}$. The corresponding energy for $\mathrm{Xe}(\mathrm{M})$ at $\hbar \omega \cong 1 \mathrm{keV}$, from the scaling relations shown in Fig.(30) and the assumption of a comparable pulse width, is actually less than $1 \mathrm{~mJ}$. The power and intensity scaling properties of a channel produced in uranium by $\mathrm{Xe}(\mathrm{L})$ radiation, at $\hbar \omega \cong 4.5 \mathrm{keV}$ are represented in Fig.(31). The calculations of the propagation of $\mathrm{Xe}(\mathrm{L})$ radiation in uranium channels show that a power of $\sim 0.75 \mathrm{PW}$ is sufficient to reach the value of $\sim 10^{27} \mathrm{~W} / \mathrm{cm}^{2}$, a value enabling entrance to the Direct Nuclear Coupling Zone illustrated in Fig.(25). As developed below, the Photon Staging concept, through its ability to produce intensities in the $10^{27} \mathrm{~W} / \mathrm{cm}^{2}-10^{29} \mathrm{~W} / \mathrm{cm}^{2}$ regime, plays a central role in both of the remaining Grand Challenge Problems that (1) involve amplification in the $\gamma$-ray region and (2) the attainment of a route to the Super-Heavy Nuclear Island of Stability. The "Photon Staging" process is the key mechanism that can provide the high intensities generally required to address these advanced technical problems. 


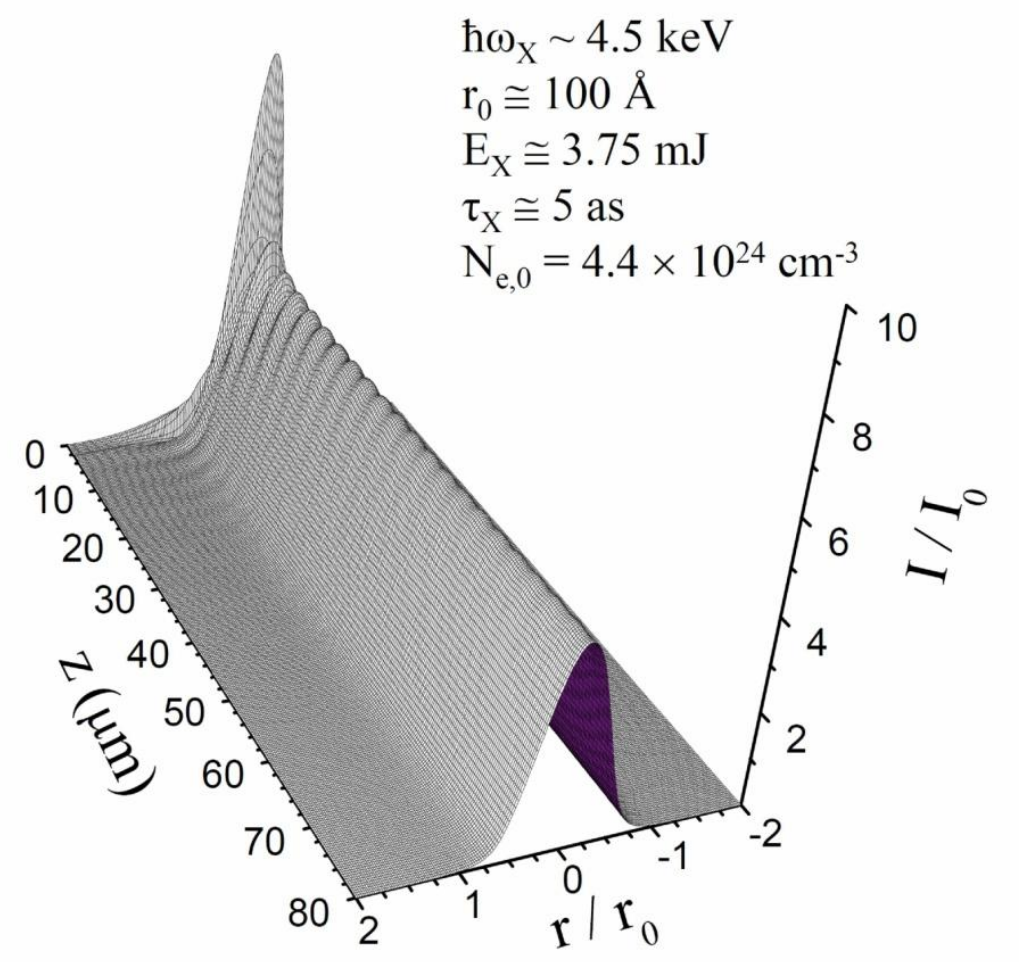

$\mathrm{I}_{\mathrm{ch}} \cong 1.0 \times 10^{27} \mathrm{~W} / \mathrm{cm}^{2}$

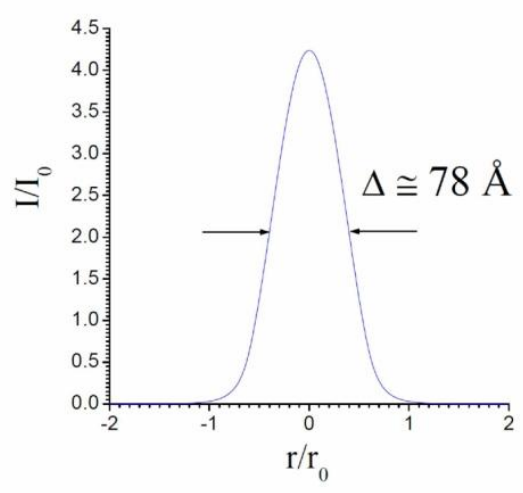

Fig.(31): Channel production in solid $\mathrm{U}$ with a $\mathrm{Xe}(\mathrm{L})$ pulse having an energy of $3.75 \mathrm{~mJ}$ and an incident power $\mathrm{P}_{0}=0.75 \mathrm{PW}$. The peak intensity produced is $\sim 10^{27} \mathrm{~W} / \mathrm{cm}^{2}$, the value suitable for the observation of vacuum harmonics.

\subsubsection{Amplification in the Gamma-Ray Region}

Excimer laser technology was the key to providing all of the data included in Fig.(1) with the exception of two points; specifically, they are the $\mathrm{NH}_{3}$ studies conducted with $\mathrm{CO}_{2}$ laser radiation [69] and the experiment conducted at the FLASH facility [47]. Hence, the definition of the Fundamental Nonlinear Domain historically rests entirely on experimental studies conducted with excimer lasers, specifically the $\mathrm{KrF}^{*}$ and $\mathrm{ArF}^{*}$ systems. Therefore, in light of the background discussed in section 3 and the conceptual extension developed explicitly below, we examine the possibility that the excimer concept could potentially be transferred into the nuclear region in a manner that would enable the amplification of $\gamma$-rays on nuclear transitions [186]. Hence, we explore below the basic question; can a "Nuclear Excimer" be achieved?

As a first step in considering this possibility, we observe that there exists a structural/dynamical analogy between excimers and a wide class of nuclear states. Consider the $\mathrm{KrF}^{*}$ system and the energy level structure illustrated in Fig.(32). The excited $\mathrm{KrF}^{*}$ state arises from the binding of the $\mathrm{Kr}^{+}$and $\mathrm{F}^{-}$ions. For both of these individual species, respectively, the removal $\left(\mathrm{Kr}^{+}\right)$ or addition $\left(\mathrm{F}^{-}\right)$of an electron affects quite weakly the orbits and energy states of the other bound electrons in the system. In the limiting case of the pure independent electron model, there is no 
influence; to wit, the orbital excitations of $\mathrm{Kr}_{\text {and }} \mathrm{Kr}^{+}$are highly comparable, both structurally and energetically. Conversely, the ground state and lowest excited states of the bound $\mathrm{KrF}^{*}$ molecule differ greatly, as apparent from the potential energy curves shown in Fig.(32). The basis of this difference is that the ionic bond formation in the excited state manifold requires a self-consistent reconfiguration of the charge density that differs grossly from those characteristic of the participating atomic states. Hence, the redistribution of the atomic charge densities associated with the bonding mechanism deviates significantly from the independent single electron picture. This feature of the dynamics of the bond formation resembles the collective interactions that are important in nuclei, particularly systems removed from closed shell configurations [186,187]. In analogy with the selfconsistent solution of the electron density associated with the $\mathrm{KrF}^{*}$ bond formation, nuclei seek a similar corresponding self-consistent arrangement of the nucleons in order to form the bound state of the system.

This structural/dynamic analogy can be explicitly illustrated by the comparison of two well documented reactions, specifically the neutron (n) induced fission of ${ }^{235} \mathrm{U}$ and the $\mathrm{KrF}^{*}$ excimer formation and radiative decay. The fission channel of ${ }^{235} \mathrm{U}$ is represented by

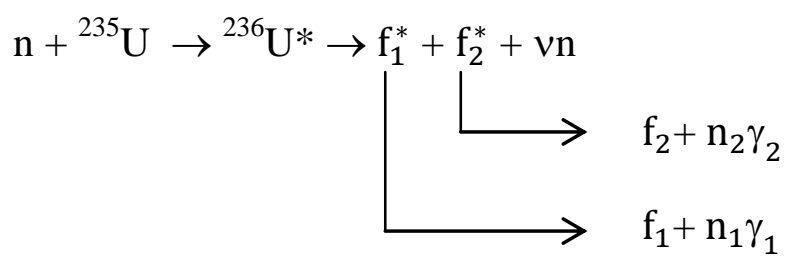

in which the unstable excited ${ }^{236} \mathrm{U}^{*}$ configuration rapidly fissions with the production of energetic excited fission fragments $\mathrm{f}_{1}^{*}$ and $\mathrm{f}_{2}^{*}$ that can further deexcite yielding gamma quanta $\gamma_{1}$ and $\gamma_{2}$ with corresponding multiplicities $n_{1}$ and $n_{2}$ together with free neutrons $n$ whose average multiplicity is $v \cong$ 2.7. The addition of a single neutron stimulates a massive rearrangement of the nucleons in the unstable ${ }^{236} \mathrm{U}^{*}$ system as it seeks a self-consistent solution to the nuclear force structure, a process that yields two fission fragments, that are generally internally excited, and a set of free neutrons. Hence, a relatively small initial perturbation arising from a single neutron is enormously amplified by the collective nuclear dynamics and produces an outcome that is a radical change in the state of the system, specifically, an energetic disintegration of the nucleus.

The dynamics of $\mathrm{KrF}^{*}$ excimer formation and radiative decay can be viewed analogously. Consider the reaction

$$
\mathrm{F}^{-}+\mathrm{Kr}^{+} \rightarrow \mathrm{KrF}^{*} \rightarrow \mathrm{Kr}+\mathrm{F}+\gamma
$$

in which the $\mathrm{F}^{-}$and $\mathrm{Kr}^{+}$ions produce the bound radiatively unstable $\mathrm{KrF}^{*}$ intermediate that rapidly decays yielding the production of the unbound neutral $\mathrm{Kr}$ and $\mathrm{F}$ atoms and the quantum $\gamma$, the mechanism shown in Fig.(32). In parallel with the fission reaction represented in Eq.(19), the large rearrangements of the charge distribution caused by the development of the self-consistent electron configuration associated with the $\mathrm{KrF}^{*}$ bond formation and the subsequent radiative decay of the $\mathrm{KrF}^{*}$ excimer lead to a radical change in the state of the system. Therefore, in both reactions, the strong reorganization arising from the self-consistent bond formation produces energetic decay of the 
entity involving the emission of particles and photons. The unstable ${ }^{236} \mathrm{U}^{*}$ system and the $\mathrm{KrF}^{*}$ excimer play analogous roles in Eqs.(19) and (20).

From this structural/dynamical analogy, it follows that the molecular "excimer" concept may be directly transferable to nuclear systems. Accordingly, many nuclei could potentially appear as attractive "excimer" candidates in the sense that their dynamic response to a relatively small initial perturbation could efficiently produce excited species capable of supporting amplification on nuclear transitions in the $\gamma$-ray region. It can be imagined that the initial interaction triggering the reaction could arise from direct electromagnetic coupling to the nucleus. As a model of this potential behavior, the selective production of excited fission fragments stimulated by an external electromagnetic interaction with a suitable nucleus, perhaps a presently unknown super-heavy system ${ }_{Z}^{A} X_{N}$ of the kind discussed below in section 4.2.3. This general process, that appears particularly prominently in the heavy fission fragment distribution [188] of many nuclei, would appear to be a fertile area of study. Following the analogy given by Eqs.(19) and (20), the illustration shown in Fig.(33) states this hypothesis as a mirror of the representation of the dynamics of the $\mathrm{KrF}^{*}$ excimer in Fig.(32).

\section{New Technology: Excimer Concept - Control of Energy}

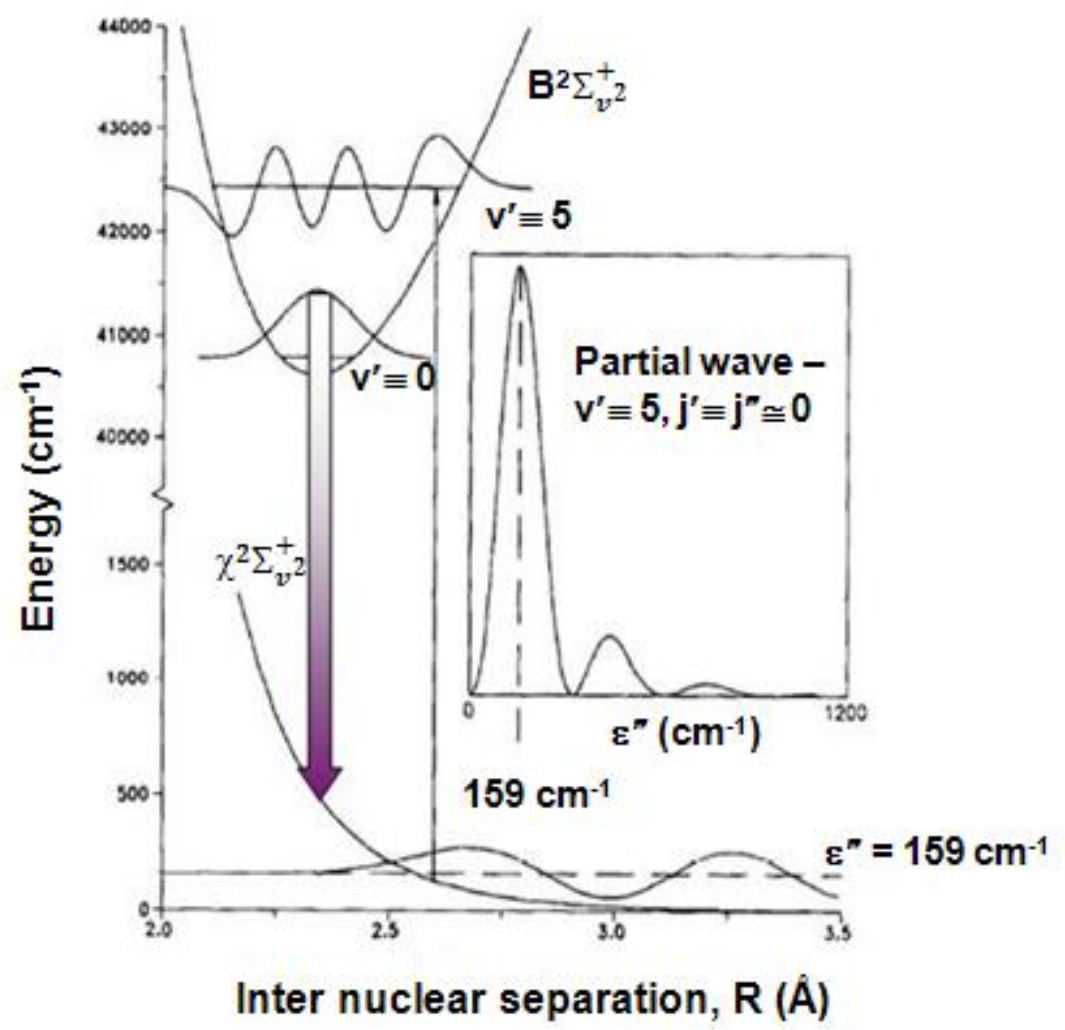

Fig.(32) : Partial energy level diagram for the $\mathrm{KrF}$ molecule illustrating the photoassociation of a $\mathrm{Kr}$ $\mathrm{F}$ collision pair (having $\approx 160 \mathrm{~cm}^{-1}$ of kinetic energy) to produce $\operatorname{KrF}^{*}\left(\mathrm{~B}, v^{\prime}=5\right.$ ). The wave functions for the $v^{\prime}=0$ and 5 levels for the $\mathrm{B}^{2} \Sigma$ state are shown and the inset gives the contribution to the photoassociation spectrum - the partial wave - for $v^{\prime}=5$. For illustrative purposes, both $\mathrm{J}^{\prime}$ and $\mathrm{J}^{\prime \prime}$ are taken to be zero in this figure. 


\section{Nuclear Excimer Concept}

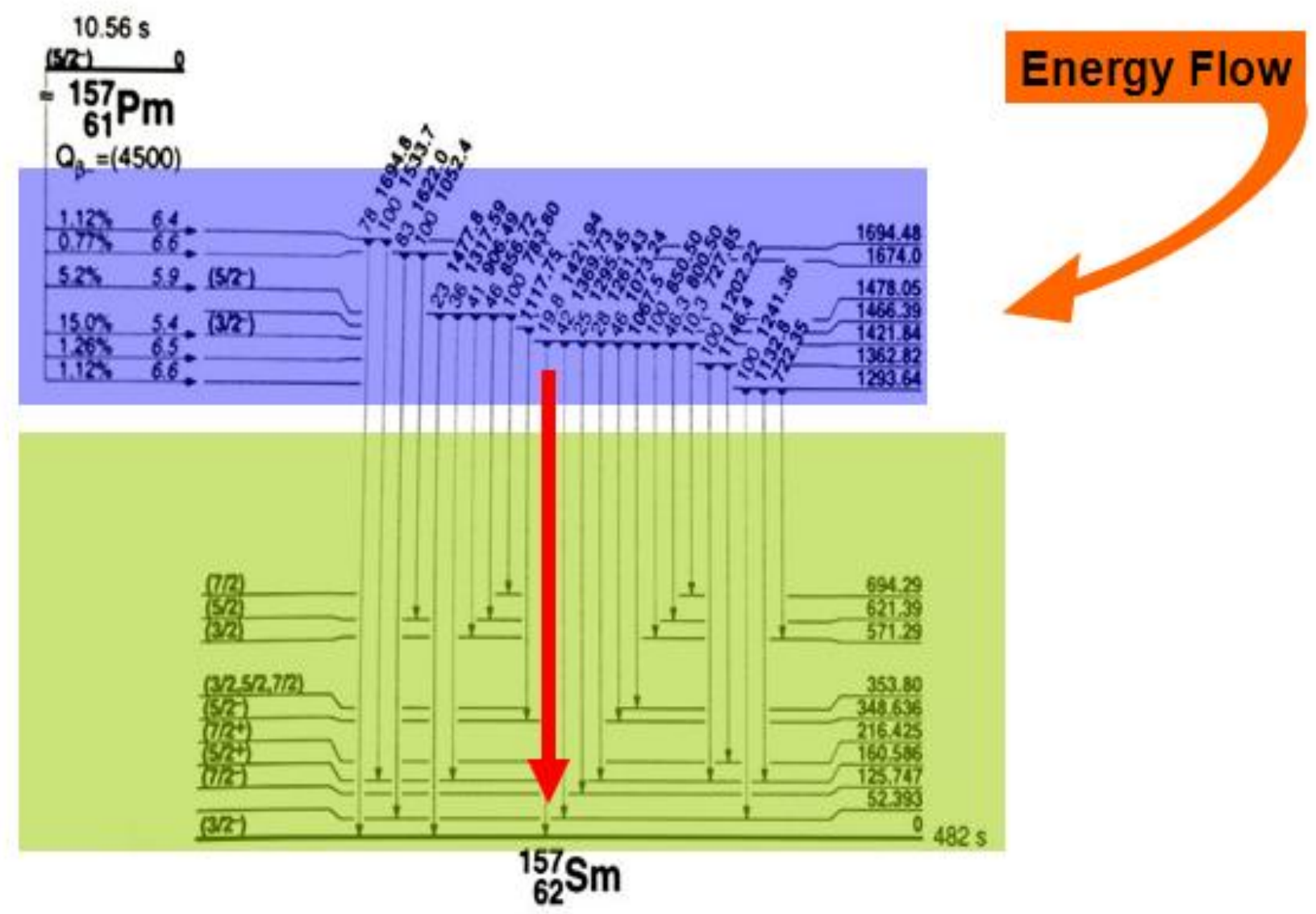

Fig.(33) : Schematic of nuclear excimer concept involving potential excimer behavior associated with fission fragments. The nuclear data shown were adapted from information in: Table of Isotopes, Vol. II, $8^{\text {th }}$ edition, Ed. By R. B. Firestone and V. S. Shirley (John Wiley \& Sons, Inc., New York, 1999).

\subsubsection{Path to Nuclear Super-Heavy Island of Stability}

The "Island of Stability", first proposed [189] by Glenn T. Seaberg in 1969, has origins stemming from the properties of the nuclear "Shell Model" initially discussed [190] by

Göppert-Mayer in 1948. At present, the predicted Island of Stability is estimated [191] to have proton number $\mathrm{Z} \cong 114$ and neutron number $\mathrm{N} \cong 184$, giving $\mathrm{A} \cong 298$ and a corresponding neutron excess of $\mathrm{N}-\mathrm{Z} \cong 70$. The exceptionally neutron-rich nuclei occupying this mass range are expected to be highly and variably distorted [192], express elevated fission energy yields $(\sim 1 \mathrm{GeV})$, and the corresponding neutral atoms are anticipated to exhibit unusual chemical properties. Essentially, everything is predicted to be anomalous. 


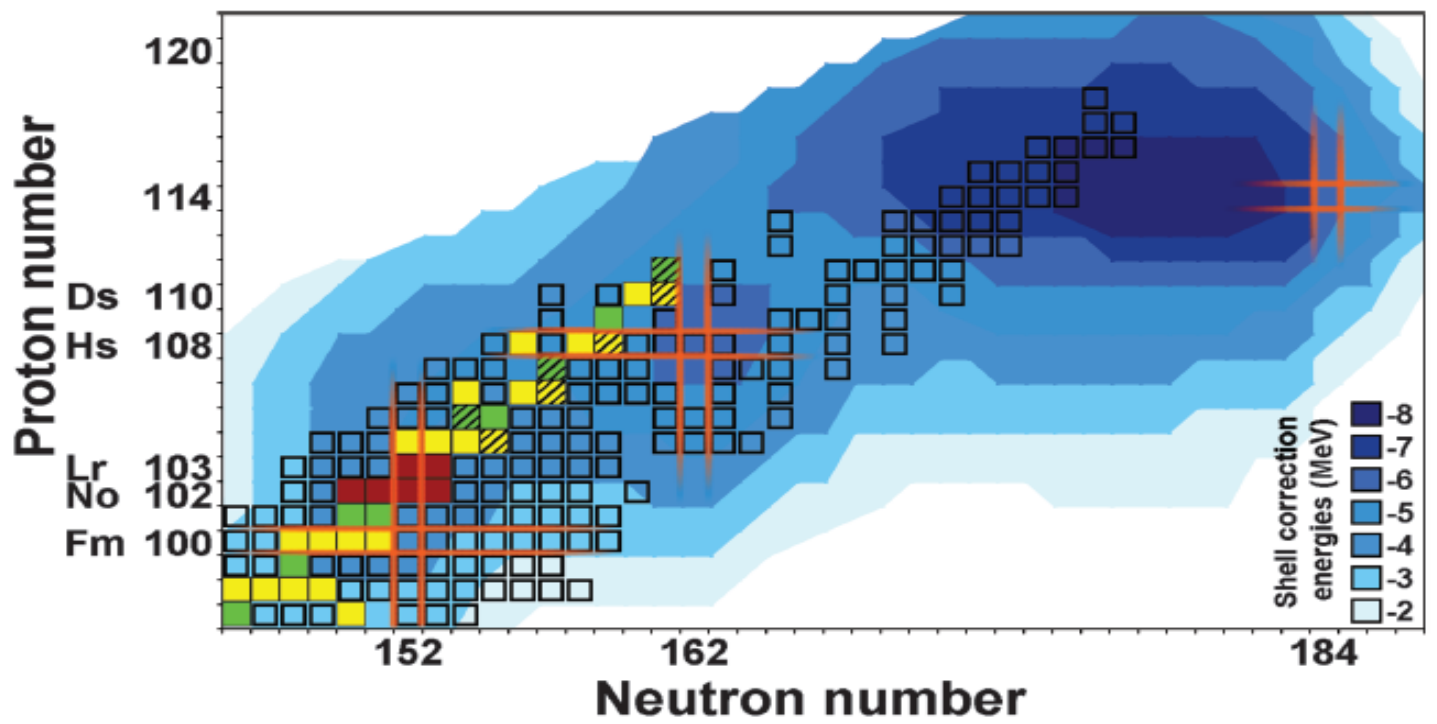

Fig.(34): Chart of nuclides above berkelium $(Z=97)$. The blue background shows the calculated shell-correction energies. The orange-shaded lines indicate known and predicted shell closures. The squares represent presently known or claimed nuclides. The nobelium and lawrencium isotopes whose masses are reported here are indicated by red squares. The yellow and green squares represent nuclides whose masses are determined by use of these new mass values, respectively, as anchor points in combination with experimental $\alpha$-decay energies. Hatched squares show nuclides with unknown or ambiguous excited states. Figure and caption adapted from Ref.[191] and used with permission.

A key step along the route to the Super-Heavy Island of Stability illustrated in Fig.(34) would be the ability to produce an abundance highly neutron-rich species from an available stable nuclear system. This desired feature naturally highlights many unusual properties of nuclear systems [193] and particularly the dynamics of the class of internal nuclear motions that produce a separation of protons and neutrons; the classic example is the giant dipole [194] in which the protons and neutrons oscillate against each other as two organized separate groups of particles. Ideally, a dynamic configuration leading selectively to enhanced proton emission from the system that would simultaneously retain the neutrons while leading to a sufficiently metastable or stable exit channel is sought. Some experimental evidence for this possibility exists in connection to the giant dipole resonance; proton emission has been observed [195] and multiple nucleon emission is experimentally known [196]. As a conceptual picture, imagine an initial maximally neutron-rich target nuclear system located just inside the boundary defined by the neutron drip-line, that is excited by direct interaction with an external field in such a way that a piece of the nuclear surface is dynamically reconfigured to pass through the proton drip-line, a step that would selectively open the door to rapid proton emission. In order to take advantage of the anisotropy of the nucleus for a deformed target system, nuclear alignment could be performed to orient the interaction. In addition, circular polarization could also be employed to communicate angular momentum in the coupling. In the $10^{27}-$ $10^{29} \mathrm{~W} / \mathrm{cm}^{2}$ range of intensity designated as the Direct Nuclear Coupling Zone in Fig.(25), simple estimates indicate a magnitude for the electromagnetic interaction energy for a system with $\mathrm{A} \cong 240$ in the range of 100-300 keV, a value comparable to level splittings in many heavy nuclei [192,197]. 


\section{Conclusions}

The research conducted over the last five decades covered by this article supports several important conclusions and points predictively toward expectations in the next half century.

\section{New Zone of Interaction}

A new zone of electromagnetic interaction has been identified. It is universal in its applicability to all materials and represents a dynamical region characterized by an enhanced coupling strength. This region is denoted as the Fundamental Nonlinear Domain and has lower limit boundaries in the Intensity (I) - Quantum Energy ( $\hbar \omega)$ plane that correspond respectively to $\mathrm{I} \geq 10^{16}$ $\mathrm{W} / \mathrm{cm}^{2}$ and $\hbar \omega \geq 10^{3} \mathrm{eV}$. The augmented interaction can be considered as an increase in the basic electromagnetic constant $\alpha$ in which $\alpha \rightarrow Z^{2} \alpha$, where $Z$ designates the number of electrons participating in a ordered response to the incident driving field. The magnitude $Z^{2} \alpha>1$ has been observed and $Z^{2} \alpha>50$ is projected to be attainable.

\section{Identification of Key Technology}

The data acquired over decades of research activity conclusively show that Excimer Laser Technology was the clear champion in the experimental campaign leading to the establishment of the Fundamental Nonlinear Domain. The basis of this role rests upon a triplet of conditions that could be simultaneously satisfied with this technology concerning the frequency $\omega$, intensity I, and power P. The achievable values of the $(\omega, \mathrm{I}, \mathrm{P})$ triplet were sufficiently high that the key processes associated with the organized electron motion leading to inner-shell excitation and the plasma channeling process could be satisfied, thereby permitting these phenomena to be controllably combined under conditions enabling rigorous and detailed experimental study.

\section{Scaling Relations and Predictions}

The derivation of important physically anchored scaling relations was achieved that enable the formulation of predictive assessments. A central relationship named "Photon Staging", that is supported with a quantitative analysis, describes the extension of the self-channeling mechanism to $\mathrm{x}$ rays in condensed matter. The predicted maximal intensities and power densities associated with these channels respectively are $\sim 10^{29} \mathrm{~W} / \mathrm{cm}^{2}$ and $\sim 10^{30} \mathrm{~W} / \mathrm{cm}^{3}$, levels of increase that represent an advance by a factor of $\sim 10^{9}-10^{10}$. It is also estimated that direct coupling to nuclei will be achievable under these conditions, a step that will enable quantitative experimental exploration of many nuclear properties and processes. The key prediction that the nuclear arena is accessible is an outcome that connects directly to two of the three Grand Challenge Problems introduced in this work.

\section{Grand Challenge Problem Formulation}

On the basis of the present experimental and theoretical understanding, a triplet of Grand Challenge Problems for future solution can be suggested. They are (1) the performance of an experimental probe of the vacuum state associated with the Dark Energy $\Omega_{\Lambda},(2)$ the attainment of the 
amplification in the $\gamma$-ray region ( $\hbar \omega \sim 1 \mathrm{MeV})$, accompanied by the discovery of a nuclear excimer, and (3) the determination of a navigable path to the projected Super-Heavy Nuclear Island of Stability. Finally, we represent the view that all three goals will be achieved in the coming decades.

\section{Projected Technological Expectations}

The anticipated technical advances projected for future capability can be framed in an approximate pictorial representation. With the parameters $\mathrm{P}, \mathrm{I}, \tau$, and $\omega$ denoting respectively the characteristic powers, intensities, time-scales, and frequencies associated with the expected developments, this picture, reading temporally from left to right, appears below in Table III.

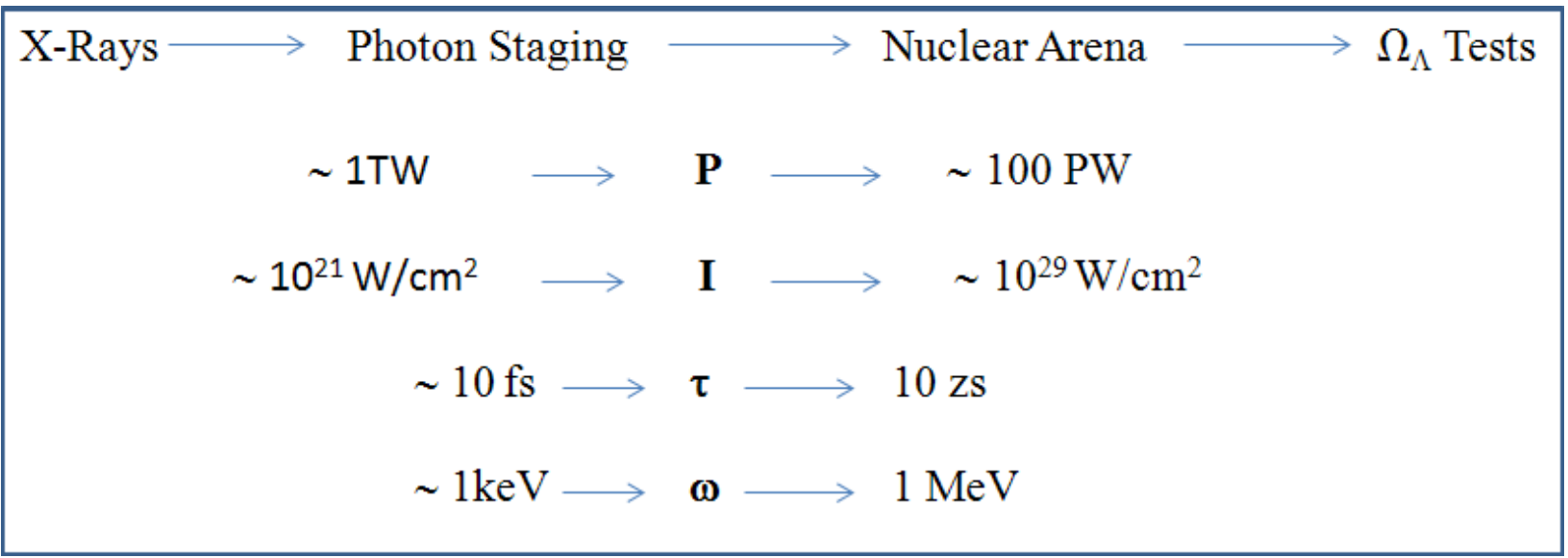

Table III: Projected technological advances.

\section{Acknowledgements:}

This research was jointly funded by DARPA on contract no. DAAD 10-01-C-00068 through the Army Research Laboratory, the University of Illinois at Chicago, Intense Field Technologies, LLC and Ultrabeam Technologies, LLC.

\section{References}

1. Franken P A, Hill A E, Peters C W and Weinreich G 1961 Generation of Optical Harmonics Phys. Rev. Lett. 7118

2. Malka V, Faure J, Gauduel Y A, Lefebvre E, Rousse A and Phuoc K T 2008 Principles and applications of compact laser-plasma accelerators Nature Phys. 4447

3. Arthur J, Anfinrud A P, Audebert A P, Bane A K, Ben-Zvi A I, Bharadwaj A V, Bionta A R, Bolton A P, Borland A M and Bucksbaum A P H 2002 Linac coherent light source (LCLS) conceptual design, SLAC, San Mateo, CA, Tech. Rep. SLAC-R593

4. Emma P, Akre R, Arthur J, Bionta R, Bostedt C, Bozek J, Brachmann A, Bucksbaum P, Coffee R, Decker F-J, Ding Y, Dowell D, Edstrom S, Fisher A, Frisch J, Gilevich S, Hastings J, Hays G, Hering P, Huang Z, Iverson R, Loos H, Messerschmidt M, Miahnahri A, Moeller S, Nuhn H-D, Pile G, Ratner D, Rzepiela J, Schultz D, Smith T, Stefan P, Tompkins H, Turner J, Welch J, White W, Wu J, Yocky G and Galayda J 2010 First lasing and operation of an angstrom-wavelength free-electron laser Nature Photon. 4641 
5. Altarelli M et al 2006 XFEL: The European X-ray free-electron laser, DESY, Hamburg, Germany, Tech. Rep.

6. Ayvazyan V, Baboi N, Bähr J, Balandin V, Beutner B, Brandt A, Bohnet I, Bolzmann A, Brinkmann R, Brovko O I, Carneiro J P, Casalbuoni S, Castellano M, Castro P, Catani L, Chiadroni E, Choroba S, Cianchi A, Delsim-Hashemi H, Di Pirro G, Dohlus M, Düsterer S, Edwards H T, Faatz B, Fateev A A, Feldhaus J, Flöttmann K, Frisch J, Fröhlich L, Garvey T, Gensch U, Golubeva N, Grabosch H-J, Grigoryan B, Grimm O, Hahn U, Han J H, Hartrott M V, Honkavaara K, Hüning M, Ischebeck R, Jaeschke E, Jablonka M, Kammering R, Katalev V, Keitel B, Khodyachykh S, Kim Y, Kocharyan V, Körfer M, Kollewe M, Kostin D, Krämer D, Krassilnikov M, Kube G, Lilje L, Limberg T, Lipka D, Löhl F, Luong M, Magne C, Menzel J, Michelato P, Miltchev V, Minty M, Möller W D, Monaco L, Müller W, Nagl M, Napoly O, Nicolosi P, Nölle D, Nuñez T, Oppelt A, Pagani C, Paparella R, Petersen B, Petrosyan B, Pflüger J, Piot P, Plönjes E, Poletto L, Proch D, Pugachov D, Rehlich K, Richter D, Riemann S, Ross M, Rossbach J, Sachwitz M, Saldin E L, Sandner W, Schlarb H, Schmidt B, Schmitz M, Schmüser P, Schneider J R, Schneidmiller E A, Schreiber H-J, Schreiber S, Shabunov A V, Sertore D, Setzer S, Simrock S, Sombrowski E, Staykov L, Steffen B, Stephan F, Stulle F, Sytchev K P, Thom H, Tiedtke K, Tischer M, Treusch R, Trines D, Tsakov I, Vardanyan A, Wanzenberg R, Weiland T, Weise H, Wendt M, Will I, Winter A, Wittenburg K, Yurkov M V, Zagorodnov I, Zambolin P and Zapfe K 2006 First operation of a free-electron laser generating $\mathrm{GW}$ power radiation at $32 \mathrm{~nm}$ wavelength Eur. Phys. J. D 37 297

7. Tiedtke K, Azima A, von Bargen N, Bittner L, Bonfigt S, Düsterer S, Faatz B, Frühling U, Gensch M, Gerth C, Guerassimova N, Hahn U, Hans T, Hesse M, Honkavaar K, Jastrow U, Juranic P, Kapitzki S, Keitel B, Kracht T, Kuhlmann M, Li W B, Martins M, Núñez T, Plönjes E, Redlin H, Saldin E L, Schneidmiller E A, Schneider J R, Schreiber S, Stojanovic N, Tavella F, Toleikis S, Treusch R, Weigelt H, Wellhöfer M, Wabnitz H, Yurkov M V and Feldhaus J 2009 The soft x-ray free-electron laser FLASH at DESY: Beamlines, diagnostics and end-stations New J. Phys. 11023029

8. Tanaka T and Shintake T 2005 SCSS X-FEL conceptual design, RIKEN Harima Institute, Hyogo, Japan, Tech. Rep.

9. Lewis C and Riley D (Eds.) 2009 X-ray Lasers 2008: Proc. 11th Int. Conf. X-Ray Lasers, Belfast, U.K., 2008. New York: Springer-Verlag 130

10. Lee J, Nam C H and Janulewicz K A (Eds.) 2011 X-ray Lasers 2010: Proc. 12th Int. Conf. XRay Lasers, Gwangju, Korea, 2010, New York: Springer-Verlag 136

11. Beckhoff B, Gottwald A, Klein R, Krumrey M, Müller R, Richter M, Scholze F, Thornagel R and Ulm G 2009 A quarter-century of metrology using synchrotron radiation by PTB in Berlin Phys. Status Solidi B 2461415

12. Borisov A B, Borovskiy A V, Korobkin V V, Prokhorov A M, Shiryaev O B, Shi X M, Luk T S, McPherson A, Solem J C, Boyer K and Rhodes C K 1992 Observation of relativistic and charge-displacement self-channeling of intense subpicosecond ultraviolet $(248 \mathrm{~nm})$ radiation in plasmas Phys. Rev. Lett. 682309

13. Borisov A B, Borovskiy A V, Shiryaev O B, Korobkin V V, Prokhorov A M, Solem J C, Luk T S, Boyer K and Rhodes C K 1992 Relativistic and charge-displacement self-channeling of intense ultrashort laser pulses in plasmas Phys. Rev. A 455830

14. Borisov A B, Shi X, Karpov V B, Korobkin V V, Solem J C, Shiryaev O B, McPherson A, Boyer K and Rhodes CK 1994 Stable self-channeling of intense ultraviolet pulses in 
underdense plasma, producing channels exceeding 100 Rayleigh lengths J. Opt. Soc. Am. B 11 1941

15. Borisov A B, Shiryaev O B, McPherson A, Boyer K and Rhodes C K 1995 Stability analysis of relativistic and charge-displacement self-channelling of intense laser pulses in underdense plasmas Plasma Phys. Control. Fusion 37569

16. Borisov A B, Longworth J W, Boyer K and Rhodes C K 1998 Stable relativistic / chargedisplacement channels in ultrahigh power density $\left(\sim 10^{21} \mathrm{~W} / \mathrm{cm}^{3}\right)$ plasmas Proc. Natl. Acad. Sci. USA 957854

17. Wagner R, Chen S-Y, Maksimchuk A and Umstadter D 1997 Electron acceleration by a laser wakefield in a relativistically self-guided channel Phys. Rev. Lett. 783125

18. Borisov A B, Cameron S, Dai Y, Davis J, Nelson T, Schroeder W A, Longworth J W, Boyer K and Rhodes C K 1999 Dynamics of optimized stable channel formation of intense laser pulses with the relativistic/charge-displacement mechanism J. Phys. B: At. Mol. Opt. Phys. 32 3511

19. Wang X, Krishnan M, Saleh N, Wang H and Umstadter D 2000 Electron acceleration and the propagation of ultrashort high-intensity laser pulses in plasmas Phys. Rev. Lett. 845324

20. Borisov A B, Cameron S, Luk T S, Nelson T R, Tassle A Van, Santoro J, Schroeder W A, Dai Y, Longworth J W, Boyer K and Rhodes C K 2001 Bifurcation mode of relativistic and charge-displacement self-channeling J. Phys. B: At. Mol. Opt. Phys. 342167

21. Pukhov A 2003 Strong field interaction of laser radiation Rep. Prog. Phys. 6647

22. Esirkepov T, Borghesi M, Bulanov S V, Mourou G and Tajima T 2004 Highly effiecient relativistic-ion generation in the laser-piston regime Phys. Rev. Lett. 92175003

23. Davis J, Borisov A B and Rhodes C K 2004 Optimization of power compression and stability of relativistic and ponderomotive self-channeling of $248 \mathrm{~nm}$ laser pulses in underdense plasmas Phys. Rev. E 70066406

24. Borisov A B, Davis J, Boyer K and Rhodes C K 2005 High-Intensity Applications of Excimer Lasers, in Excimer Laser Technology, D. Basting and G. Marowsky, eds., Berlin:SpringerVerlag p 373

25. Davis J, Borisov A B, Boyer K and Rhodes C K 2005 Multikilovolt Channelled X-Ray Propagation in Water J. Phys. B: At. Mol. Opt. Phys. 38 L309

26. Borisov A B, McCorkindale J C, Poopalasingam S, Longworth J W and Rhodes C K 2013 Comparative Analysis of Stable Ultrahigh Power-Density $248 \mathrm{~nm}$ Channels Formed in $\mathrm{Kr}$ and Xe Cluster Targets J. Phys. B: At. Mol. Opt. Phys. 46185601

27. Luk T S, Pummer H, Boyer K, Shahidi M, Egger H and Rhodes C K 1983 Anomalous Collision-Free Multiple Ionization of Atoms with Intense Picosecond Ultraviolet Radiation Phys. Rev. Lett. 51110

28. Boyer K and Rhodes C K 1985 Atomic Inner-Shell Excitation Induced by Coherent Motion of Outer-Shell Electrons Phys. Rev. Lett. 541490

29. Szöke A and Rhodes C K 1986 Theoretical Model of Inner-Shell Excitation by Outer-Shell Electrons Phys. Rev. Lett. 56720

30. Rhodes C K 1985 Multiphoton Ionization of Atoms Science 2291345

31. Boyer K, Luk T S, Solem J C and Rhodes C K 1989 Kinetic Energy Distributions of Ionic Fragments Produced by Subpicosecond Multiphoton Ionization of $\mathrm{N}_{2}$ Phys. Rev. A 391186

32. Gibson G, Luk T S, McPherson A, Boyer K and Rhodes C K 1989 Observations of a New Inner-Orbital Molecular Transition at $55.8 \mathrm{~nm}$ in $\mathrm{N}_{2}{ }^{2+}$ Produced by Multiphoton Coupling Phys. Rev. A 402378 
33. McPherson A, Luk T S, Thompson B D, Boyer K and Rhodes C K 1993 Multiphoton-Induced X-Ray Emission and Amplification from Clusters Appl. Phys. B 57337

34. McPherson A, Thompson B D, Borisov A B, Boyer K and Rhodes C K 1994 MultiphotonInduced X-Ray Emission at 4-5 keV from Xe Atoms with Multiple Core Vacancies Nature 370631

35. Boyer K and Rhodes C K 1994 Superstrong Coherent Multi-Electron Intense-Field Interaction J. Phys. B: At. Mol. Opt. Phys. 27 L633

36. Schroeder W A, Nelson T R, Borisov A B, Longworth J, Boyer K and Rhodes C K 2001 An Efficient Selective Collisional Ejection Mechanism for Inner-Shell Population Inversion in Laser-Driven Plasmas J. Phys. B: At. Mol. Opt. Phys. 34297

37. Borisov A B, McPherson A, Thompson B D, Boyer K and Rhodes C K 1995 Ultrahigh Power Compression for X-Ray Amplification: Multiphoton Cluster Excitation Combined with Nonlinear Channeled Propagation, J. Phys. B: At. Mol. Opt. Phys. 282143

38. Borisov A B, Song X, Frigeni F, Koshman Y, Dai Y, Boyer K and Rhodes C K 2003 Ultrabright Multikilovolt Coherent Tunable X-Ray Source at $\lambda \sim 2.71-2.93 \AA$ J. Phys. B: At. Mol. Opt. Phys. 363433

39. Borisov A B, Song X, Koshman Y, Dai Y, Boyer K and Rhodes C K 2003 Saturated Multikilovolt X-Ray Amplification with Xe Clusters: Single-Pulse Observation of Xe(L) Spectral Hole-Burning J. Phys. B: At. Mol. Opt. Phys. 36 L285

40. Borisov A B, Racz E, Zhang P, McCorkindale J C, Khan S F, Poopalasingam S, Zhao J and Rhodes C K 2008 Realization of the conceptual ideal for x-ray amplification J. Phys. B: At. Mol. Opt. Phys. 41105602

41. Kondo K, Borisov A B, Jordan C, McPherson A, Schroeder W A, Boyer K and Rhodes C K 1997 Wavelength Dependence of Multiphoton-Induced Xe(M) and Xe(L) Emissions from Xe Clusters J. Phys. B: At. Mol. Opt. Phys. 302707

42. Borisov A B, Song X, Frigeni F, Dai Y, Koshman Y, Schroeder W A, Boyer K and Rhodes C K 2002 Ultraviolet-infrared Wavelength Scalings for Strong Field Induced L-Shell Emissions from Kr and Xe Clusters J. Phys. B: At. Mol. Opt. Phys. 35 L461

43. Petrov G M, Davis J, Velikovich A L, Kepple P C, Dasgupta A, Clark R W, Borisov A B, Boyer K and Rhodes C K 2005 Modeling of Clusters in a Strong 248-nm Laser Field by a Three-Dimensional Relativistic Molecular Dynamic Model Phys. Rev. E 71036411

44. Richter M, Amusia M Ya, Bobashev S V, Feigl T, Juranic P N, Martins M, Sorokin A A and Tiedtke K 2009 Extreme Ultraviolet Laser Excites Atomic Giant Resonance Phys. Rev. Lett. 102163002

45. Saalmann U and Rost J-M 2002 Ionization of Clusters in Strong X-Ray Laser Pulses Phys. Rev. Lett. 89143401

46. Boyer K, Jara H, Luk T S, McIntyre I A, McPherson A, Rosman R and Rhodes C K 1987 Limiting Cross Sections for Multiphoton Coupling Revue Phys. Appl. 221793

47. Richter M 2011 Atomic plasma excitations in the field of a soft x-ray laser J. Phys. B: At. Mol. Opt. Phys. 44075601

48. Richardson V, Costello J T, Cubaynes D, Düsterer S, Feldhaus J, Hart H W van der, Juranić P, Li W B, Meyer M, Richter M, Sorokin A A and Tiedke K 2010 Two-Photon Inner-Shell Ionization in the Extreme Ultraviolet Phys. Rev. Lett. 105013001

49. Rudek B et al. 2012 Ultra-efficient ionization of heavy atoms by intense X-ray free-electron laser pulses Nature Photonics 6858 
50. Borisov A B, Longworth J W, McPherson A, Boyer K and Rhodes C K 1996 Dynamical orbital collapse drives super x-ray emission J. Phys. B: At. Mol. Opt. Phys. 29247

51. Honda H, Miura E, Katsura K, Takahashi E and Kondo K 2000 Evidence for wavelength dependence of Xe M-shell emission from clusters Phys. Rev. A 61023201

52. Boyer K, Thompson B D, McPherson A and Rhodes C K 1994 Evidence for Coherent Electron Motions in Multiphoton X-Ray Production from $\mathrm{Kr}$ and Xe Clusters J. Phys. B: At. Mol. Opt. Phys. 274373

53. Thompson B D, McPherson A, Boyer K and Rhodes C K 1994 Multi-Electron Ejection of Inner-Shell Electrons through Multiphoton Excitation of Clusters J. Phys. B: At. Mol. Opt. Phys. 274391

54. McPherson A, Boyer K and Rhodes C K 1994 X-Ray Superradiance from Multiphoton Excited Clusters J. Phys. B: At. Mol. Opt. Phys. 27 L637

55. Borisov A B, McPherson A, Boyer K and Rhodes C K 1996 Z- $\lambda$ Imaging of Xe(M) and Xe(L) Emissions from Channeled Propagation of Intense Femtosecond $248 \mathrm{~nm}$ Pulses in a Xe Cluster Target J. Phys. B: At. Mol. Opt. Phys. 29 L113

56. Borisov A B, McPherson A, Boyer K and Rhodes C K 1996 Intensity Dependence of the Multiphoton-Induced Xe(L) Spectrum Produced by Subpicosecond $248 \mathrm{~nm}$ Excitation of Xe Clusters J. Phys. B: At. Mol. Opt. Phys. 29 L43

57. McPherson A, Cobble J, Borisov A B, Thompson B D, Omenetto F, Boyer K and Rhodes C K 1997 Evidence of Enhanced Multiphoton (248 nm) Coupling from Single-Pulse Energy Measurements of Xe(L) Emission Induced from Xe Clusters J. Phys. B: At. Mol. Opt. Phys. 30 L767

58. Marowsky G and Rhodes Ch K 1996 Hohle Atome und die Kompression von Licht in Plasmakanänalen Physikalische Blätter 52991

59. Borisov A B, McPherson A, Thompson B D, Boyer K and Rhodes C K 1997 Multielectron Motions Induced in Clusters and Molecules in Self-Trapped Channels, In: Multiphoton Processes 1996 (Institute of Physics Conference Series Number 154), ed. P. Lambropoulos and H. Walther (IOP Publishing, Bristol, U.K., 1997) p 1

60. Schroeder W A, Omenetto F G, Borisov A B, Longworth J W, McPherson A, Jordan C, Boyer K, Kondo K and Rhodes C K 1998 Pump Laser Wavelength-Dependent Control of the Efficiency of Kilovolt X-Ray Emission from Atomic Clusters J. Phys. B: At. Mol. Opt. Phys. 315031

61. Borisov A B, Song X, Zhang P, Dasgupta A, Davis J, Kepple P C, Dai Y, Boyer K and Rhodes C K 2005 Amplification at $\lambda \sim 2.8 \AA$ on $\mathrm{Xe}(\mathrm{L})(2 \overline{\mathrm{s}} 2 \overline{\mathrm{p}})$ Double-Vacancy States Produced by $248 \mathrm{~nm}$ Excitation of Xe Clusters in Plasma Channels J. Phys. B: At. Mol. Opt. Phys. 383935

62. Boyer K, Borisov A B, Song X, Zhang P, McCorkindale J C, Khan S, Dai Y, Kepple P C, Davis J and Rhodes C K 2005 Explosive Supersaturated Amplification on 3d $\rightarrow 2 \mathrm{p} \mathrm{Xe}(\mathrm{L})$ Hollow Atom Transitions at $\lambda \sim$ 2.7-2.9 Å J. Phys. B: At. Mol. Opt. Phys. 383055

63. Borisov A B, Song X, Zhang P, McCorkindale J C, Khan S F, Poopalasingam S, Zhao J, Dai Y and Rhodes C K 2007 Double Optimization of Xe(L) Amplifier Power Scaling at $\lambda \sim 2.9 \AA$ J. Phys. B: At. Mol. Opt. Phys. 40 F131

64. Borisov A B, Zhang P, Racz E, McCorkindale J C, Khan S F, Poopalasingam S, Zhao J and Rhodes C K 2007 Temperature Enhancement of Xe(L) X-Ray Amplifier $(\lambda \sim 2.9 \AA \AA)$ Emission J. Phys. B: At. Mol. Opt. Phys. 40 F307 
65. Borisov A B, Racz E, Khan S F, Poopalasingam S, McCorkindale J C, Zhao J, Boguta J, Longworth J W and Rhodes C K 2010 Power Scaling of the Xe(L) Amplifier at $\lambda \sim 2.8 \AA$ into the Petawatt Regime J. Phys. B: At. Mol. Opt. Phys. 43015402

66. Borisov A B, Racz E, Khan S F, Poopalasingam S, McCorkindale J C, Zhao J, Fontanarosa J B, Dai Y, Boguta J, Longworth J W and Rhodes C K 2010 Spatially Resolved Observation of the Spectral Hole Burning in the Xe(L) Amplifier on Single $(2 \overline{\mathrm{p}})$ and Double $(2 \overline{\mathrm{s}} 2 \overline{\mathrm{p}})$ Vacancy $3 \mathrm{~d} \rightarrow 2 \mathrm{p}$ Transitions in the $2.62 \AA<\lambda<2.94 \AA$ Range J. Phys. B: At. Mol. Opt. Phys. 43045402

67. Borisov A B, Khan S F, Racz E, Poopalasingam S, McCorkindale J C, Zhao J, Fontanarosa J B, Dai Y, Longworth J W and Rhodes C K 2012 Observation of a Curve Crossing Mechanism in the Field Ionization of Inner-Shell Excited Single $\mathrm{Xe}^{33+}(2 \overline{\mathrm{p}})$ and Double $\mathrm{Xe}^{34+}(2 \overline{\mathrm{s}} 2 \overline{\mathrm{p}})$ Vacancy States IEEE J. Quant. Electr. 48806

68. Patel C K N 1964 Continuous-Wave Laser Action on Vibrational-Rotational Transitions of $\mathrm{CO}_{2}$ Phys. Rev. Lett. 136 A1187

69. Bischel W K, Kelly P J and Rhodes C K 1976 High-Resolution Doppler-Free Two-Photon Spectroscopic Studies of Molecules II: the $v_{2}$ Bands of ${ }^{14} \mathrm{NH}_{3}$ Phys. Rev. A 131829

70. Brau C A and Ewing J J 1975 354-nm laser action on XeF Appl. Phys. Lett. 27435

71. Pummer H, Egger H and Rhodes Ch K 1979 High-Spectral-Brightness Excimer Systems, in: Excimer Lasers, edited by Ch. K. Rhodes, Topics in Applied Physics, Vol. 30 (SpringerVerlag, Berlin/Heidelberg/ New York, 1979) p.217

72. Kligler D J and Rhodes C K 1978 Observation of Two-Photon Excitation of the $\mathrm{H}_{2} \mathrm{E}, \mathrm{F}^{1} \Sigma_{\mathrm{g}}{ }^{+}$ State Phys. Rev. Lett. $\mathbf{4 0} 309$

73. Kligler D J, Bokor J and Rhodes C K 1980 Collisional and Radiative Properties of the $\mathrm{H}_{2} \mathrm{E}$, $\mathrm{F}^{1} \Sigma_{\mathrm{g}}{ }^{+}$State Phys. Rev. A 21607

74. Egger H, Luk T S, Boyer K, Muller D F, Pummer H, Srinivasan T and Rhodes C K 1982 Picosecond, Tunable ArF* Excimer Laser Source Appl. Phys. Lett. 411032

75. Harakeh M N and van der Wonde A 2001 Giant Resonances (Clarendon Press, Oxford, 2001)

76. Boyer K, Gibson G, Jara H, Luk T S, McIntyre I A, McPherson A, Rosman R, Solem J C and Rhodes And C K 1988 Corresponding Aspects of Strong-Field Multiquantun Processes and Ion-Atom Collisions IEEE Transactions on Plasma Science 16541

77. Lofthus A and Krupenie P H 1977 The Spectrum of Molecular Nitrogen J. Phys. Chem. Ref. Data 6113

78. Herzberg G 1950 Molecular Spectra and Molecular Structure. I. Spectra of Diatomic Molecules, 2nd ed. (Van Nostrand Reinhold, New York, 1950)

79. Ågren H 1981 On the Interpretation of Molecular Valence Auger Spectra J. Chem. Phys. 75 1267

80. Borisov A B and Rhodes C K 2014 Stable Power Compression with Efficient Relativistic UV Channel Formation in Cluster Targets, in: Planar Waveguides and Other Confined Geometries: Theory, Technology, Production, and Novel Applications, ed. G. Marowsky, Springer Series in Optical Sciences

81. McPherson A, Luk T S, Thompson B D, Borisov A B, Shiryaev O B, Chen X, Boyer K and Rhodes C K 1994 Multiphoton Induced X-ray Emission from Kr Clusters on M-Shell ( 100 $\AA$ ) and L-Shell ( $6 \AA$ ) Transitions Phys. Rev. Lett. 721810

82. Boyer K, Chen X, McPherson A, Thompson B D and Rhodes C K 1994 Multiphoton X-Ray Generation from Clusters: Analysis of Energy Transfer Scaling, Proceedings of the Sixth 
International Conference: Multiphoton Processes, D. K. Evans and S. L. Chin (eds.), (World Scientific, Singapore, 1994) p 467

83. Lee P H Y, Casperson D E and Schappert G T 1989 Search for Multiphoton-Induced InnerShell Excitation Phys. Rev. A 401363

84. Rhodes C K 1994 New Advances Toward A Kilovolt X-Ray Amplifier Optics \& Photonics News 58

85. Borisov A B, McCorkindale J C, Poopalasingam S, Longworth J W and Rhodes C K 2013 Demonstration of $\operatorname{Kr}(\mathrm{L})$ Amplification at $\lambda=7.5 \AA$ from Kr Clusters in Self-Trapped Plasma Channels J. Phys. B: At. Mol. Opt. Phys. 46155601

86. Wolford M F, Myers M C, Giuliani J L, Sethian J D and Hegeler F 2006 Electra: repetitively pulsed 700 J, 100 ns electron beam pumped KrF laser Proc. SPIE 6101, Laser Beam Control and Applications, 61011M (February 23, 2006)

87. Borisov A B, McCorkindale J C, Poopalasingam S, Longworth J W and Rhodes C K 2013 New Directions in High Intensity Interactions J. Phys. B: At. Mol. Opt. Phys. http://iopscience.iop.org/0953-4075/labtalk-article/54941

88. Åberg T and Howat G 1982 Theory of the Auger Effect, in Handbunch der Physik Band XXXI, Korpulskeln und Strahlung in Materie I, Bandherausgegeber W. Mehlhorn (Berlin: Springer-Verlag, 1982) p 469

89. Chen M H, Crasemann B and Mark H 1979 Relativistic radiationless transition probabilities for atomic K- and L-shells At. Data Nucl. Data Tables 2413

90. Krause M O and Oliver J H 1979 Natural Widths of Atomic K and L Levels, Ka X-Ray Lines and Several KLL Auger Lines J. Phys. Chem. Ref. Data 8329

91. Jitschin W, Stötzel R, Papp T, Sankar M and Doolen G D 1995 Coster-Kronig yields of the ${ }^{54}$ Xe L subshells measured through synchrotron photoionization Phys. Rev. A 52977

92. McGuire E J 1975 Auger and Coster-Kronig transitions Atomic Inner-shell Processes I, Ionization and Transition Probabilities ed B Crasemann (New York:Academic, 1975) p 293

93. McGuire E J 1971 Atomic L-shell Coster-Kronig, Auger and radiative rates and fluorescence yields for Na-Th Phys. Rev. A 3587

94. Crasemann B, Chen M H and Kostroun V O 1971 Auger and Coster-Kronig transition probabilities to the atomic 2s state and theoretical $\mathrm{L}_{1}$ fluorescence yields Phys. Rev. A 42161

95. Sevier K D 1972 Low Energy Electron Spectrometry (New York: Wiley-Interscience, 1972)

96. McGuire E J 1971 L-shell Auger and Coster-Kronig Electron Spectra Phys. Rev. A 31801

97. Fedotov A M, Narozhny N B, Mourou G and Korn G 2010 Limitations on the Attainable Intensity of High Power Lasers Phys. Rev. Lett. 105080402

98. Heisenberg W and Euler H 1936 Folgerungen aus der Diracschen Theorie des Positrons Zeitschr. Phys. 98714

99. Schwinger J 1951 On Guage Invariance and Vacuum Polarization Phys. Rev. 82664

100. Borisov A B, Song X, Zhang P, Boyer K and Rhodes C K 2006 The Nuclear Era of Laser Interactions: New Milestones in the History of Power Compression, in Lasers and NucleiApplications of Ultrahigh Intensity Lasers in Nuclear Science, edited by H. Schwoerer, J. Magill, B. Beleites, Lect. Notes Phys. (Springer-Verlag, Berlin/Heidelberg, 2006) 694 p 3

101. Borisov A B, Racz E, Khan S F, Poopalasingam S, McCorkindale J C, Zhao J, Boguta J, Longworth J W, Rhodes C K and 2009 The Nuclear Epoch of Laser Interactions, The Fourth International Symposium on Atomic Cluster Collisions(ISAACC 2009), Edited by A. V. Solov'yov and E. Surdutovich, AIP Conf. Proc. 119742 
102. Borisov A B, McCorkindale J C, Poopalasingam S, Longworth J W and Rhodes C K 2013 Reaching Vacuum Harmonic Generation and Approaching the Schwinger Limit with X-rays Contrib. Plasma Phys. 53179

103. Boyer K, Luk T S and Rhodes C K 1988 Possibility of Optically Induced Nuclear Fission Phys. Rev. Lett. 60557

104. Ledingham K W D et al 2000 Photonuclear Physics when a Multiterawatt Laser Pulse Interacts with Solid Targets Phys. Rev. Lett. 84899

105. Cowan T E et al 2000 Photonuclear Fission from High Energy Electrons from Ultraintense Laser-Solid Interactions Phys. Rev. Lett. 84903

106. Lasers and Nuclei-Applications of Ultrahigh Intensity Lasers in Nuclear Science, edited by $\mathrm{H}$. Schwoerer, J. Magill, B. Beleites, Lect. Notes Phys. 694 (Springer-Verlag, Berlin/Heidelberg, 2006)

107. Abella I D 1962 Optical Double-Photon Absorption in Cesium Vapor Phys. Rev. Lett. 9453

108. Clark M W, Schneider D, DeWitt D, McDonald J W, Bruch R, Safronova U I, Tolstikhina I Yu and Schuch R 1993 Xe L and M X-ray emission following Xe $\mathrm{e}^{44-48+}$ ion impact on $\mathrm{Cu}$ surfaces Phys. Rev. A 473983

109. Plechaty E F, Cullen D E and Howerton R J 1981 Tables and Graphs of Photon-Interaction Cross Sections from $0.1 \mathrm{keV}$ to $100 \mathrm{MeV}$ Derived from the LLL Evaluated-Nuclear-Data Library 6268 UCRL-50400 (Gaithersburg, MD: National Institute of Standards and Technology)

110. Rhodes C K 1986 Ordered Many-Electron Motions in Atoms and X-Ray Lasers, in Giant Resonances in Atoms, Molecules, and Solids, edited by J. P. Connerade, J. M. Esrera, and R. C. Kamatak, NATO ASI Series B, Physics Vol. 151 (Plenum Publishing Corporation, New York, 1986) p 533

111. Viefhaus J, Braune M, Korica S, Reinköster A, Rolles D and Becker U 2005 Auger cascades versus direct double Auger: relaxation processes following photoionization of the $\mathrm{Kr} 3 \mathrm{~d}$ and Xe 4d, 3d inner shells J. Phys. B: At. Mol. Opt. Phys. 383885

112. Pubst S and Santra R 2013 Strong-Field Many-Body Physics and the Giant Enhancement in the High-Harmonic Spectrum of Xenon Phys. Rev. Lett. 111233005

113. Griffin D C et al 1984 Electron-impact ionization in the xenon isonuclear sequence Phys. Rev. A 291729

114. Marowsky G and Rhodes C K 1995 Hohle Atome: Eine nene Form Von hochangeregter materie, Nene Zürcher Zeiturg 25442.

115. Mitchel A and Zemansky M 1961 Absorption lines and measurements of the lifetime of the resonance state chapter III in Resonance Radiation and Excited Atoms (Cambridge UP) 92

116. Rhodes C K, Brau C A, Gallagher A and Hoff P W 1983 Excimer Lasers, Topics in Applied Physics (Springer, Berlin, Heidelberg)

117. Pätzel R and Stamm U 2005 Excimer lasers for microlithography Chapter 6 In: Excimer Laser Technology, ed. by Basting D, Marowsky G (Springer, Berlin, Heidelberg) 98

118. Smith P W 1989 Pulsed power techniques for discharge pumped visible and ultraviolet excimer lasers IEE Colloquium on Pulsed Power Applications, Digest 135, INSPEC Acc. 33993791311

119. Mathew D, Bastiaens H M J, Boller K-J and Peters P J M 2006 Current filamentation in discharge-excited $\mathrm{F}_{2}$-based excimer laser gas mixtures Appl. Phys. Lett. 88101502 
120. von Bergmann H, Rebhan U and Stamm U 2005 Design and Technology of Excimer Lasers chapter 4 In: Excimer Laser Technology ed. by Basting D, Marowsky G (Springer, Berlin, Heidelberg) 47

121. Zhao Q, Szatmári S and Schäfer F P 1988 Gain dynamics of XeF and subpicosecond pulse generation at $351 \mathrm{~nm}$ Appl. Phys. B 47325

122. Corkum P B and Taylor R S 1982 Picosecond amplification and kinetic studies of XeCl IEEE J. Quantum Electron. 181962

123. Szatmári S and Schäfer F P 1987 Comparative study of the gain dynamics of $\mathrm{XeCl}$ and $\mathrm{KrF}$ with subpicosecond resolution J. Opt. Soc. Am. B 41943

124. Szatmári S and Schäfer F P 1984 Picosecond gain dynamics of KrF* Appl. Phys. B 33219

125. Taylor A J, Gibson R B and Roberts J P 1988 Picosecond gain dynamics in KrF amplifiers Appl. Phys. Lett. 52773

126. Taylor A J, Gosnell T R and Roberts J P 1990 Ultrashort-pulse energy-extraction measurements in $\mathrm{XeCl}$ amplifiers Opt. Lett. 15118

127. Bucksbaum P H, Bokor J, Storz R H and White J C 1982 Amplification of ultrashort pulses in krypton fluoride at $248 \mathrm{~nm}$ Opt. Lett. 7399

128. Nishioka H, Kuranishi H, Ueda K and Takuma H 1989 UV saturable absorber for short-pulse KrF laser systems Opt. Lett. 14692

129. Kühnle G, Teubner U and Szatmári S 1990 Amplified spontaneous emission in short-pulse excimer amplifiers Appl. Phys. B 5171

130. Shaw M J, Key M H and Ross I 1990 in: Reference Papers European High Performance Laser Facility, Eds: Fabre E and Key M H Rutherford Appleton Laboratory 253

131. Szatmári S, Kühnle G, Endoh A, Schäfer F P, Jasny J, Lee Y W, Jethwa J, Teubner U and Kovács G. 1990 Technical Proposal for the ELF 100 J/100 fs KrF-Laser System SIMBA

132. Almási G, Szatmári S and Simon P 1992 Optimized operation of short-pulse KrF amplifiers by off-axis amplification Opt. Commun. 88231

133. Greene A E and Brau C A 1978 Theoretical Studies of UV-Preionized Transverse Discharge $\mathrm{KrF}$ and ArF Lasers IEEE J. Quantum Electron. 14951

134. Sze R C and Loree T R 1978 Experimental studies of a $\mathrm{KrF}$ and ArF discharge laser IEEE J. Quantum Electron. 14944

135. Frantz L M and Nodvik J S 1963 Theory of pulse propagation in a laser amplifier J. Appl. Phys. 342346

136. Tillemann M M and Jacob J H 1987 Short pulse amplification in the presence of absorption Appl. Phys. Lett. 50121

137. Szatmári S 1992 Terawatt-class hybrid dye/excimer lasers Topics in Applied Physics 70 Dye Lasers 25 Years, Stuke, M. (ed.), Berlin: Springer, 129

138. Szatmari S, Marowsky G and Simon P 2007 Femtosecond excimer lasers and their applications Landolt-Börnstein - Group VIII Advanced Materials and Technologies Vol. 1.B.1 Laser Physics and Applications - Laser Systems, G. Herziger, H. Weber, and R. Poprawe, eds., (Springer Berlin Heidelberg,), 215

139. Szatmári S, Almási G and Simon P 1991 Off-axis amplification scheme for short-pulse amplifiers Appl. Phys. B $\mathbf{5 3} 82$

140. Rhodes C K 1979 Excimer Lasers, Berlin, Heidelberg: Springer-Verlag

141. McIntyre I A and Rhodes C K 1991 High power ultrafast excimer lasers J. Appl. Phys. 69 R1

142. Szatmári S and Simon P 1993 Interferometric multiplexing scheme for excimer amplifiers Opt. Commun. 98181 
143. Szatmári S, Bakonyi Z and Simon P 1997 Active spatial filtering of laser beams Opt. Commun. 134199

144. Simon P, Nagy T and Szatmári S 1998 Nonlinear spectral filtering of femtosecond pulses Opt. Commun. 145155

145. Brabec T and Krausz F Rev. 2000 Intense few-cycle laser fields: frontiers of nonlinear optics Mod. Phys. 72545

146. Corkum P B and Krausz F 2007 Attosecond science Nature Physics 3381

147. Pittman M, Ferré S, Chambaret J P and Chéruaux G 2002 Design and characterization of a near diffraction limited femtosecond $100 \mathrm{TW}-10 \mathrm{~Hz}$ high intensity laser system Appl. Phys. B 74529

148. Kalachnikov M P, Karpov V, Schönnagel H and Sandner W 2002 100-terawatt titaniumsapphire laser system Laser Physics 12368

149. Aoyama M, Yamakawa K, Akahane Y, Ma J, Inoue N, Ueda H and Kiriyama H 2003 0.85PW, 33-fs Ti:sapphire laser Opt. Lett. 281594

150. Bahk S-W, Chvykov V, Kalintchenko G, Maksimchuk A, Mourou G A, Saleh N and Yanovsky V 2004 Generation, Amplitude and Phase Characterization of $10^{21} \mathrm{~W} / \mathrm{cm}^{2}$ Intensity Ultrafast Optics IV 95331 (Springer-Verlag, Berlin)

151. Kalashnikov M P, Risse E, Schonnagel H, Husakou A, Herrmann J and Sandner W, 2004 Characterization of a nonlinear filter for the front-end of a high contrast double-CPA Ti:sapphire laser Opt. Express 125088

152. Jullien A, Albert O, Burgy F, Harmoniaux G, Rousseau J-P, Chambaret J-P, Augé-Rochereau $\mathrm{F}$, Chériaux G, and Etchepare J $200510^{-10}$ temporal contrast for femtosecond ultraintense lasers by cross-polarized wave generation Opt. Lett. 30920

153. Canova L, Kourtev S, Minkovski N, Jullien A, Lopez-Martens R, Albert O and Saltiel S M 2008 Efficient generation of cross-polarized femtosecond pulses in cubic crystals with holographic cut orientation Appl. Phys. Lett. 92231102

154. Kourtev S, Minkovski N, Canova L, Jullien A, Albert O and Saltiel SM 2009 Improved nonlinear cross-polarized wave generation in cubic crystals by optimization of the crystal orientation J. Opt. Soc. Am. B 261269

155. Kalashnikov M P, Osvay K, Priebe G, Ehrentraut L, Steinke S and Sandner W 2012 Temporal contrast of high intensity laser systems above 1011 with double CPA technique AIP Conf. Proc. 1462108

156. Ricci A, Jullien A, Rousseau J-P, Liu Y, Houard A, Ramirez P, Papadopoulos D, Pellegrina A, Georges P, Duon F, Forget N and Lopez-Martens R 2013 Energy-scalable temporal cleaning device for femtosecond laser pulses based on cross-polarized wave generation Rev. Sci. Instrum. 84043106

157. Kaganovich D, Penano J R, Helle M H, Gordon D F, Hafizi B and Ting A 2013 Origin and control of the subpicosecond pedestal in femtosecond laser systems Opt. Lett. 383635

158. Wharton K B, Boley C D, Komashko A M, Rubenchik A M, Zweiback J, Crane J, Hays G, Cowan T E and Dimitre T 2001 Effects of nonionizing prepulses in high-intensity laser-solid interactions Phys. Rev. E 64025401

159. Földes I B, Bakos J S, Gál K, Juhász Z, Kedves M Á, Kocsis G, Szatmári S and Veres G 2000 Properties of High Harmonics Generated by Ultrashort UV Laser Pulses on Solid Surfaces Laser Phys. 10264

160. Kapteyn H, Murnane M, Szoke A and Falcone R 1991 Prepulse energy suppression for highenergy ultrashort pulses using self-induced plasma shuttering Opt. Lett. 16490 
161. Thaury C, Quéré F, Geinder J P, Levy A, Ceccotti T, Monot P, Bougeard M, Réau F, D'Oliveira P, Audebert P, Marjoribanks R and Martin P H 2007 Plasma mirrors for ultrahighintensity optics Nature Physics 3424

162. Földes I B, Csáti D, Szücs F L and Szatmári S 2010 Plasma mirror and temperature evolution for short pulse KrF lasers Rad. Effects \& Defects in Solids 165429

163. Békési J, Marowsky G, Szatmári S and Simon P 2001 A 100 mJ table-top short pulse amplifier for $248 \mathrm{~nm}$ using interferometric multiplexing Zeitschrift für Physikalische Chemie 2151543

164. Obenschain S P, Sethian J D and Schmitt A J 2009 A Laser Based Fusion Test Facility Fusion Science and Technology 56594

165. Herman F and Skillman S 1963 Atomic Structure Calculations (Prentice-Hall, Englewood Cliffs, NJ, 1963)

166. Carlson T A, Nestor C W, Jr, Wasserman N and McDowell J D 1970 Calculated Ionization Potentials for Multiply Charged Ions Atomic Data 263

167. McDonald C R, Orlando G, Vampa G and Brabec T 2013 Tunnel Ionization Dynamics of Bound Systems in Laser Fields: How Long Does It Take for a Bound Electron to Tunnel? Phys. Rev. Lett. 111090405

168. Pfeiffer A N, Cirelli C, Smolarski M, Dimitrovski D, Abu-Samha M, Madsen L B and Keller U 2012 Attoclock reveals natural coordinates of the laser-induced tunnelling current flow in atoms Nature Physics $\mathbf{8} 76$

169. Ruf H, Handschin C, Cireasa R, Thiré N, Ferré A, Petit S, Descamps D, Mével E, Constant E, Blanchet V, Fabre B and Mairesse Y 2013 Inhomogeneous High Harmonic Generation in Krypton Clusters Phys. Rev. Lett. 110083902

170. The Nuclear Fission Process, edited by C. Wagemans (CRC Press, Inc., Boca Raton, FL, 1991)

171. Negele J W, Koonin S E, Möller P, Nix J R and Sierk A J 1978 Dynamics of Induced Fission Phys. Rev. C 171098

172. Butsch R, Hofman D J, Montoya C P, Paul P and Thoennessen M 1991 Time scales for fusion-fission and quasifission from giant dipole resonance decay Phys. Rev. C 441515

173. Harakeh M N and Van der Woude A 2001 Giant Resonances: Fundamental High-Frequency Modes of Nuclear Excitation (Oxford, Clarendon Press, 2001) p 33

174. Abusara H, Afanasjev A V and Ring P 2012 Fission barriers in covariant density functional theory: Extrapolation to superheavy nuclei Phys. Rev. C 85024314

175. Allen S W, Rapetti D A, Schmidt R W, Ebling H, Morris R G and Fabian A C 2008 Improved Constraints on Dark Energy from Chandra X-ray Observations of the Largest Relaxed Galaxy Clusters Mon. Not. R. Astron. Soc. 383879

176. Marioni C and Bussi A 2010 A Geometric Measure of Dark Energy with Pairs of Galaxies Nature 468539

177. Sauter F 1931 Über das Verhalten eines Elektrons im homogenen elektrischen Feld nach der relativistischen Theorie Diracs Zeitschrift für Physik A, Hadrons and Nuclei 69742

178. Bulanov S S, Esirkepov T Zh, Thomas A G R, Koga J K and Bulanov S V 2010 Schwinger Limit Attainability with Extreme Power Lasers Phys. Rev. Lett. 105220407

179. Bulanov S S, Mur V D, Narozhny N B, Nees J and Popov V S 2010 Multiple Colliding Electromagnetic Pulses: A Way to Lower the Threshold of e+e- Pair Production from Vacuum Phys. Rev. Lett. 104220404 
180. Heinzl T, Liesfield B, Amthor K-U, Schwoerer H, Sauerbrey R and Wipf A 2006 On the observation of vacuum birefringence Optics Communications 267318

181. Lundin J, Marklund M, Lundstörm E and Brodin G 2006 Analysis of four-wave mixing of high-power lasers for the detection of elastic photon-photon scattering Phys. Rev. A 74 043821

182. Marklund M and Lundin J 2009 Quantum vacuum experiments using high intensity lasers Eur. Phys. J. D 55319

183. Fedotov A M and Narozhny N B 2007 Generation of harmonics by a focused laser beam in the vacuum Phys. Lett. A $\mathbf{3 6 2} 1$

184. Borisov A B and Rhodes C K 2011 Hard X-Ray/Gamma-Ray Photon Staging Design Model University of Illinois at Chicago, Laboratory for X-Ray Microimaging and Bioinformatics, Internal Report

185. Rhodes C K, Boguta J, Borisov A B, Khan S F, Longworth J W, McCorkindale J C, Poopalasingam S, Racz E and Zhao J 2010 Reaching the Schwinger Limit With X-Rays, Proceedings of International Conference on Physics in Intense Fields PIF 2010: 24-26 November 2010, KEK, Tsukuba, Japan

186. Eisenberg J M and Greiner W 1987 Nuclear Theory, Volume 1, Nuclear Models, Third Edition (North-Holland, Amsterdam, 1987)

187. Bohr A and Mottelson B R 1975 Nuclear Structure, Volume II, (W. H. Benjamin, Inc., Reading, MA, 1975)

188. Knitter H-H, Brosa U and Budtz-Jørgensen C 1991 Neutron and Gamma Emission in Fission, in Wagemans C, The Nuclear Fission Process (CRC Press, Boca Raton, FL, 1991) p 497

189. Seaborg G T 1969 Prospects for Further Considerable Extension of the Periodic Table Journal of Chemical Education 46626

190. Göppert-Mayer M 1948 On Closed Shells in Nuclei Phys. Rev. 74235

191. Minaya Ramirez E, Ackermann D, Blaum K, Block M, Droese C, Düllmann Ch E, Dworschak M, Eibach M, Eliseev S, Haettner E, Herfurth F, Heßberger F P, Hofmann S, Ketelaer J, Marx G, Mazzocco M, Nesterenko D, Novikov Yu N, Plaß W R, Rodríguez D, Scheidenberger C, Schweikhard L, Thirolf P G and Weber C 2012 Direct Mapping of Nuclear Shell Effects in the Heaviest Elements Science 3371207

192. Ćwiok S, Heenen P H and Nazarewicz W 2005 Shape Coexistence and Triaxiality in the Superheavy Nuclei Nature $\mathbf{4 3 3} 705$

193. Gunst J, Litvinov Y A, Keitel C H and Pállfy A 2014 Dominant Secondary Nuclear Photoexcitation with the X-Ray Free-Electron Laser Phys. Rev. Lett. 112082501

194. Goldhaber M and Teller E 1948 On Nuclear Dipole Vibrations Phys. Rev. 741046

195. Hunyadi M, Hashimoto H, Li T, Akimune H, Fujimura H, Fujiwara M, Gácsi Z, Garg U, Hara K, Harakeh M N, Hoffman J, Itoh M, Murakami T, Nakanishi K, Nayak B K, Okumura S, Sakaguchi H, Terashima S, Uchida M, Yasuda Y and Yosoi M 2009 Proton decay from the isoscalar giant dipole resonance in ${ }^{58} \mathrm{Ni}$ Phys. Rev. C 80044317

196. Ermakov A N, Makarenko I V, Orlin V N, Ishkhanov B S and Kapitonov I M 2011 MultiParticle Photonuclear Reactions behind Giant Dipole Resonance Journal of the Korean Physical Society 591936

197. Just M, Goerlach U, Habs D, Metag V and Specht H J 1980 Combined analysis of the promptfission, delayed-fission, photofission and angular-distribution data from ${ }^{234,236} \mathrm{U}$ and ${ }^{238,240} \mathrm{Pu}$, in: Proc. IAEA Symposium on Physics and Chemistry of Fission, IAEA, Vienna, vol.1, p. 71, Bernan Press (PA). 